\title{
OUTER ACTIONS OF A DISCRETE AMENABLE GROUP ON APPROXIMATELY FINITE DIMENSIONAL FACTORS II, THE III $_{\lambda}$-CASE, $\lambda \neq 0$
}

\author{
YOSHIKAZU KATAYAMA and MASAMICHI TAKESAKI
}

(Dedicated to the memory of Gert K. Pedersen)

\begin{abstract}
To study outer actions $\alpha$ of a group $G$ on a factor $\mathcal{M}$ of type $\mathrm{III}_{\lambda}, 0<\lambda<1$, we study first the cohomology group of a group with the unitary group of an abelian von Neumann algebra as a coefficient group and establish a technique to reduce the coefficient group to the torus $T$ by the Shapiro mechanism based on the groupoid approach. We then show a functorial construction of outer actions of a countable discrete amenable group on an AFD factor of type $\mathrm{III}_{\lambda}$, sharpening the result in $[17, \S 4]$. The periodicity of the flow of weights on a factor $\mathcal{M}$ of type III $_{\lambda}$ allows us to introduce an equivariant commutative square directly related to the discrete core. But this makes it necessary to introduce an enlarged group $\operatorname{Aut}(\mathcal{M})_{\mathrm{m}}$ relative to the modulus homomorphism $\mathrm{m}=\bmod : \operatorname{Aut}(\mathcal{M}) \mapsto \mathrm{R} / T^{\prime} \mathrm{Z}$. We then discuss the reduced modified HJR-exact sequence, which allows us to describe the invariant of outer action $\alpha$ in a simpler form than the one for a general AFD factor: for example, the cohomology group $\mathrm{H}_{\mathrm{m}, \mathfrak{F}}^{\text {out }}(G, N, \mathrm{~T})$ of modular obstructions is a compact abelian group. Making use of these reductions, we prove the classification result of outer actions of $G$ on an AFD factor $\mathcal{M}$ of type $\mathrm{III}_{\lambda}$.
\end{abstract}

$\S 0$. Introduction

$\S 1$. Groupoid Cohomology

§2. Model Construction II

§3. Reduction of Invariants for the Case of Type $\mathrm{III}_{\lambda}, 0<\lambda<1$

$\S 4$. Outer actions of a Countable Discrete Amenable Group on an AFD Factor of Type $\mathrm{III}_{\lambda}, 0<\lambda<1$

§5. Outer actions of a Countable Discrete Amenable Group on an AFD Factor of Type $\mathrm{III}_{1}$

\section{Introduction}

Thanks to these celebrated results: [2], [3], [4], [6] (see [27], [28], [29] for detail), the investigations of cocycle conjugacy classification of actions of a

Received September 29, 2005. 
group $G$ on AFD factor have been finished with a series of works: [5], [7], [12], [13], [14], [15], [19], [20], [24], [25] and [26] when $G$ is discrete amenable or compact abelian. Its related topics of outer conjugacy classification of outer acition began with [7], [12] and [20] and we give its outer conjugacy classification in [16], [17] without any restriction.

In this article, we continue our investigation of outer conjugacy classification of outer actions, say $\alpha$, of a countable discrete group $G$ on a factor $\mathcal{M}$ of type III $_{\lambda}, 0<\lambda \leq 1$. Since the characteristic square of the factor $\mathcal{M}$ in [14], [15] takes a simpler form, the outer conjugacy invariants for an outer action $\alpha$ of $G$ takes a simpler form than the general case which was completed in the last work, [17]. But this does not mean that our task was completed in the last work. We have to reduce the general theory to the seemingly easy case of $\mathrm{III}_{\lambda}$, which requires more work. Once the work is completed, we see that the final form in this particular case is simpler. A major hurdle for this is the fact that the association of a discrete core $\widetilde{\mathcal{M}}_{d}$, a factor of type $\mathrm{II}_{\infty}$, to a factor $\mathcal{M}$ of type $\mathrm{III}_{\lambda}$ is not a functor. Accordingly, the group $\operatorname{Aut}(\mathcal{M})$ does not act canonically on $\widetilde{\mathcal{M}}_{d}$. The obstruction to this is the presence of the modulus $\bmod (\alpha)$ of $\alpha \in \operatorname{Aut}(\mathcal{M})([8])$. Instead, an enlarged group $\operatorname{Aut}(\mathcal{M})_{\mathrm{m}}$, which is a central extension of $\operatorname{Aut}(\mathcal{M})$ by the integer group Z, acts on $\widetilde{\mathcal{M}}_{d}$. The Shapiro machinery helps to relate the characteristic square to the reduced characteristic square consisting of all Borel groups with compact abelian groups, in fact, the circle group, on the crucial corner. To capture the Shapiro machine, we need to work with groupoid cohomology to get a clear and natural picture, which is done in the first section. An interesting feature of the case of type $\mathrm{III}_{\lambda}, 0<\lambda<1$, is that the canonical two cocycle associated with the exact sequence ([2]):

$$
0 \longrightarrow \mathrm{Z} \stackrel{\times T^{\prime}}{\longrightarrow} \mathrm{R} \longrightarrow \mathrm{R} / T^{\prime} \mathrm{Z} \longrightarrow 0, \quad T^{\prime}=-\log \lambda,
$$

which comes from the Gauss symbol $[x], x \in \mathbf{R}$, the integer $n$ such that $n \leq x<n+1$, enters naturally to the theory. We will use the notation $\{\dot{s}\}_{T^{\prime}}$ for the cross-section:

$$
\dot{s}=s+T^{\prime} \mathrm{Z} \in \mathrm{R} / T \mathrm{Z}^{\prime} \mapsto\{\dot{s}\}_{T^{\prime}}=s-T^{\prime}\left[\frac{s}{T^{\prime}}\right] \in\left[0, T^{\prime}\right), \quad s \in \mathrm{R} .
$$

The case of type $\mathrm{III}_{1}$ is even easier as its general theory is already reduced.

Toward the completion of this article, the authors have received support from the Erwin Schrödinger Institute and the Department of Mathematics, University of Rome, La Sapienza, where they visited to work together. The authors would like to express here their gratitude to these institutions and Professors K. Schmidt and S. Doplicher for their invitation and the hospitality extended to them, which made this collaboration possible and enjoyable. 
To keep the size of this article down, we postpone the discussion of examples to the subsequent paper, [18], in which the third cohomology groups of easy cases are computed and the invariants of outer actions of such groups are identified from their raw data.

\section{Groupoid Cohomology}

\section{Shapiro's Mechanism and Dimension Shifting}

Cohomology theory in groups began with [9]. The reserach of cohomology in field of operator algebra appeared in [12], [20], [22] and [23] which are related with our project. Here we extend its theory, especially dimension shifting, to a groupoid for the later use.

Let $\mathscr{G}$ be a groupoid with $X=\mathscr{G}^{(0)}$ the space of units. We note that whenever we consider a Borel groupoid, locally compact groupoid or measured groupoid, we mean by a map always a Borel map or a measurable map. For a measured groupoid, we ignore the difference on a null set. By a $\mathscr{G}$-module $\mathscr{A}$, we mean a field of groups $x \in X \mapsto A(x) \in$ Grp such that

i) Each $g \in \mathscr{G}$ gives rise to an isomorphism $\alpha_{g} \in \operatorname{Iso}(A(\mathrm{~s}(g)), A(\mathrm{r}(g))$;

ii) The family of isomorphisms $\left\{\alpha_{g}: g \in \mathscr{G}\right\}$ satisfies the chain rule:

$$
\alpha_{g h}=\alpha_{g} \circ \alpha_{h}, \quad(g, h) \in \mathscr{G}^{(2)} ;
$$

iii) If $x=g \in \mathscr{G}^{(0)}$, then $\alpha_{g}=\mathrm{id} \in \operatorname{Aut}(A(x))$.

When each $A(x), x \in X$, is commutative, then $\mathscr{A}$ is called commutative or abelian. We assume that $\mathscr{A}$ is commutative. An $n$-cochain, $n=0,1,2, \ldots$, means a function

$$
\xi:\left(g_{1}, \ldots, g_{n}\right) \in \mathscr{G}^{(n)} \mapsto \xi\left(g_{1}, \ldots, g_{n}\right) \in A\left(\mathrm{r}\left(g_{1}\right)\right)
$$

The set $\mathrm{C}_{\alpha}^{n}(\mathscr{G}, \mathscr{A})$ of $n$-cochains forms a group relative to the pointwise product. The coboundary map $\partial_{n}: \mathrm{C}_{\alpha}^{n}(\mathscr{G}, \mathscr{A}) \mapsto \mathrm{C}_{\alpha}^{n+1}(\mathscr{G}, \mathscr{A})$ is defined by

$$
\begin{aligned}
& \left(\partial_{n} \xi\right)\left(g_{0}, g_{1}, \ldots, g_{n}\right)=\alpha_{g_{0}}\left(\xi\left(g_{1}, \ldots, g_{n}\right)\right) \\
& \times \prod_{k=0}^{n-1} \xi\left(g_{0}, g_{1}, \ldots, g_{k-2}, g_{k-1} g_{k}, g_{k+1}, g_{k+2}, \ldots, g_{n}\right)^{(-1)^{k-1}} \\
& \quad \times \xi\left(g_{0}, g_{1}, \ldots, g_{n-1}\right)^{(-1)^{n-1}} \in A\left(\mathrm{r}\left(g_{0}\right)\right) .
\end{aligned}
$$

As usual, we have

$$
\partial_{n} \circ \partial_{n-1}: \mathrm{Z}_{\alpha}^{n-1}(\mathscr{G}, \mathscr{A}) \mapsto\{1\} \subset \mathrm{Z}_{\alpha}^{n+1}(\mathscr{G}, \mathscr{A}) .
$$


We often suppress the suffix $n$ of $\partial_{n}$. Each element of the kernel $\operatorname{Ker}\left(\partial_{n}\right)$, denoted by $Z_{\alpha}^{n}(\mathscr{G}, \mathscr{A})$, is called an $n$-cocycle and each element of the image $\operatorname{Im}\left(\partial_{n-1}\right)$, denoted by $\mathrm{B}_{\alpha}^{n}(\mathscr{G}, \mathscr{A})$, is called an $n$-coboundary. The quotient group $\mathrm{Z}_{\alpha}^{n}(\mathscr{G}, \mathscr{A}) / \mathrm{B}_{\alpha}^{n}(\mathscr{G}, \mathscr{A})$ is called the $n$-th cohomology group of $\mathscr{G}$ and written $\mathrm{H}_{\alpha}^{n}(\mathscr{G}, \mathscr{A})$ (See [1], [30] and [31] for the related topics).

For $n=0$, we set

$$
\begin{aligned}
& \mathrm{H}_{\alpha}^{0}(\mathscr{G}, \mathscr{A})=\mathrm{Z}_{\alpha}^{0}(\mathscr{G}, \mathscr{A}) \\
& \quad=\left\{\xi: x \in X \mapsto \xi(x) \in A(x) \text { such that } \xi(\mathrm{r}(g))=\alpha_{g}(\xi(\mathrm{s}(g)), g \in \mathscr{G}\} .\right.
\end{aligned}
$$

For $n=1, \mathrm{Z}_{\alpha}^{1}(\mathscr{G}, \mathscr{A})$ consists of all maps $\xi: g \in \mathscr{G} \mapsto \xi(g) \in A(\mathrm{r}(g))$ such that

$$
\xi(g h)=\xi(g) \alpha_{g}(\xi(h)), \quad(g, h) \in \mathscr{G}^{(2)},
$$

and $\mathrm{B}_{\alpha}^{1}(\mathscr{G}, \mathscr{A})$ consists of all those $\xi \in \mathrm{Z}_{\alpha}^{1}(\mathscr{G}, \mathscr{A})$ such that

$$
\xi(g)=\alpha_{g}\left(\eta(\mathrm{s}(g)) \eta(\mathrm{r}(g))^{-1}, \quad g \in \mathscr{G},\right.
$$

for some $\eta: x \in X \mapsto \eta(x) \in A(x)$. Each $\xi \in Z_{\alpha}^{1}(\mathscr{G}, \mathscr{A})$ gives rise to the perturbation ${ }_{\xi} \alpha$ of the action $\alpha$ on $\mathscr{A}$ given in the following fashion:

$$
\xi \alpha_{g}(u)=\xi(g) \alpha_{g}(u) \xi(g)^{-1} \in A(\mathrm{r}(g)), \quad u \in A(\mathrm{~s}(g)), \quad g \in \mathscr{G} .
$$

If $\xi \in \mathrm{B}_{\alpha}^{1}(\mathscr{G}, \mathscr{A})$, then the perturbed action ${ }_{\xi} \alpha$ is conjugate to the original action $\alpha$ under the group $\operatorname{Int}(\mathscr{A})$ of "inner" automorphisms of $\mathscr{A}$.

For $n=2$, each element $\xi \in Z_{\alpha}^{2}(\mathscr{G}, \mathscr{A})$ is an $\mathscr{A}$-valued function on $\mathscr{G}^{(2)}$ such that

$$
\xi(g, h) \xi(g h, k)=\alpha_{g}(\xi(h, k)) \xi(g, h k) \in A(\mathrm{r}(g)), \quad(g, h, k) \in \mathscr{G}^{(3)} .
$$

The cocycle $\xi$ is a coboundary if and only if there exists a map $\eta: g \in \mathscr{G} \mapsto$ $\eta(g) \in A(\mathrm{r}(g))$ such that

$$
\xi(g, h)=\alpha_{g}(\eta(h)) \eta(g h)^{-1} \eta(g), \quad(g, h) \in \mathscr{G}^{(2)} .
$$

Each cocycle $\xi \in \mathrm{Z}_{\alpha}^{2}(\mathscr{G}, \mathscr{A})$ gives rise to the twisted semi-direct product groupoid:

$$
\mathscr{H}=\mathscr{A} \rtimes_{\alpha, \xi} \mathscr{G}=\{(u, g) \in \mathscr{A} \times \mathscr{G}: u \in A(\mathrm{r}(g)), g \in \mathscr{G}\}
$$

such that

$$
\begin{gathered}
\mathscr{H}^{(2)}=\left\{(u, g ; v, h) \in \mathscr{H} \times \mathscr{H}:(g, h) \in \mathscr{G}^{(2)}\right\} \\
\mathrm{r}(u, g)=\mathrm{r}(g), \quad \mathrm{s}(u, g)=\mathrm{s}(g) ; \\
(u, g)(v, h)=\left(u \alpha_{g}(v) \xi(g, h), g h\right), \quad(u, g ; v, h) \in \mathscr{H}^{(2)} .
\end{gathered}
$$


The original $\mathscr{G}$-module $\mathscr{A}$ is then viewed as a normal subgroupoid of $\mathscr{H}$ and the original groupoid $\mathscr{G}$ is then identified with the quotient groupoid: $\mathscr{G}=\mathscr{H} / \mathscr{A}$. The action $\alpha$ of $\mathscr{G}$ on $\mathscr{A}$ is then nothing but the conjugation:

$$
\alpha_{g}(u)=\left(1_{y}, g\right)(u, x)\left(1_{y}, g\right)^{-1} \in A(y), \quad g=(y, g, x) \in \mathscr{G},
$$

where $\mathrm{r}(g)=y, \mathrm{~s}(g)=x$ and $1_{y}$ is the identity of $A(y)$. If $\xi=\partial(\eta)$, then the map:

$$
\mathfrak{S}_{\eta}: g \in \mathscr{G} \mapsto\left(\eta(g)^{-1}, g\right) \in \mathscr{H}
$$

is an injective homomorphism of the groupoid $\mathscr{G}$ into $\mathscr{H}$ which decomposes $\mathscr{H}$ into a semi-direct product:

$$
\mathscr{H} \cong \mathscr{A} \rtimes_{\alpha} \mathscr{G}
$$

For $n=0,1,2$, the $\mathscr{G}$-module $\mathscr{A}$ does not have to be commutative to define $\mathrm{H}_{\alpha}^{n}(\mathscr{G}, \mathscr{A})$ as long as one is ready to give up the group structure on the cohomology space $\mathrm{H}_{\alpha}^{n}(\mathscr{G}, \mathscr{A}), n=0,1,2$. For $n=2$, the cocycle identity, however, should be replaced by:

$$
\alpha_{g}(\xi(h, k)) \xi(g, h k)\{\xi(g, h) \xi(g h, k)\}^{-1}=1, \quad(g, h, k) \in \mathscr{G}^{(3)},
$$

and the equivalence $\xi \equiv \xi^{\prime}$ of two cocycles $\xi$ and $\xi^{\prime}$ is defined by the existence of $\eta: g \in \mathscr{G} \mapsto \eta(g) \in A(\mathrm{r}(g))$ such that

$$
\xi^{\prime}(g, h)=\eta(g) \alpha_{g}(\eta(h)) \xi(g, h) \eta(g h)^{-1}, \quad(g, h) \in \mathscr{G}^{(2)} .
$$

If the groupoid $\mathscr{G}$ is a topological groupoid and $\mathscr{A}$ admits a topological structure such that all the operations are continuous, then we request that cocycles are all Borel. To demand the continuity on cocycles is too restrictive as seen in the group case. If $\mathscr{G}$ is a measured groupoid, then all the identities mean to hold almost everywhere relative to the relevant measure class.

Proposition 1.1. Let $\mathscr{A}$ be a $\mathscr{G}$-module. For each $y \in X=\mathscr{G}^{(0)}$, let $B(x)$ be the set of all $A(x)$-valued functions on $\mathscr{G}_{x}=\mathrm{s}^{-1}(x)$ and set

$$
\mathscr{B}=\bigcup_{x \in X}^{\bullet} B(x)=\{b: \mathscr{G} \mapsto \mathscr{A} \text { such that } b(g) \in A(x), g=(y, g, x) \in \mathscr{G}\}
$$

For each $g=(y, g, x) \in \mathscr{G}$, define the map $\beta_{g}: B(x) \mapsto B(y)$ in the following fashion:

$$
\left(\beta_{g} b\right)(h)=\alpha_{g}(b(h g)) \in A(y), \quad b \in B(x), \quad(h, g) \in \mathscr{G}^{(2)} .
$$


Then for each $n=2,3, \ldots$,

$$
\mathrm{H}_{\beta}^{n}(\mathscr{G}, \mathscr{B})=\{1\} .
$$

More explicitly, if $\xi \in \mathrm{Z}_{\beta}^{n}(\mathscr{G}, \mathscr{B})$, then $\eta \in \mathrm{C}_{\beta}^{n-1}(\mathscr{G}, \mathscr{B})$ defined by $\eta\left(g ; g_{1}, g_{2}, \ldots, g_{n-1}\right)$

$$
=\alpha_{g}^{-1}\left(\xi\left(\mathrm{r}(g) ; g, g_{1}, \ldots, g_{n-1}\right)\right) \in A(\mathrm{~s}(g)), \quad\left(g, g_{1}, \ldots, g_{n-1}\right) \in \mathscr{G}^{(n)} .
$$

gives

$$
\xi=\partial \eta
$$

Proof. The cocycle identity:

$$
\begin{aligned}
1=\beta_{g_{0}}\left(\xi\left(g_{1}, \ldots, g_{n}\right)\right) & \prod_{i=1}^{n} \xi\left(g_{0}, g_{1}, g_{2}, \ldots, g_{i-2}, g_{i-1} g_{i}, g_{i+1}, \ldots, g_{n}\right)^{(-1)^{i}} \\
& \times \xi\left(g_{0}, \ldots, g_{n-1}\right)^{(-1)^{n}}, \quad\left(g_{0}, g_{1}, \ldots, g_{n}\right) \in \mathscr{G}^{(n+1)}
\end{aligned}
$$

gives

$$
\begin{array}{r}
\xi\left(g_{1}, \ldots, g_{n}\right)=\beta_{g_{0}}^{-1}\left(\prod_{i=1}^{n} \xi\left(g_{0}, g_{1}, g_{2}, \ldots, g_{i-2}, g_{i-1} g_{i}, g_{i+1}, \ldots g_{n}\right)^{(-1)^{i-1}}\right. \\
\left.\times \xi\left(g_{0}, \ldots, g_{n-1}\right)^{(-1)^{n-1}}\right)
\end{array}
$$

which means that for each $\left(g, g_{1}, \ldots, g_{n}\right) \in \mathscr{G}^{(n+1)}$ with $g=g_{0}$,

$$
\begin{aligned}
\xi\left(g ; g_{1}, \ldots, g_{n}\right) & \\
=\alpha_{g_{0}}^{-1} & \left(\prod_{i=1}^{n} \xi\left(g g_{0}^{-1} ; g_{0}, g_{1}, g_{2}, \ldots, g_{i-2}, g_{i-1} g_{i}, g_{i+1}, \ldots, g_{n}\right)^{(-1)^{i-1}}\right. \\
\quad & \left.\quad \xi\left(g g_{0}^{-1} ; g_{0}, \ldots, g_{n-1}\right)^{(-1)^{n}}\right) \\
=\alpha_{g}^{-1} & \left(\prod_{i=1}^{n} \xi\left(\mathrm{r}(g) ; g, g_{1}, g_{2}, \ldots, g_{i-2}, g_{i-1} g_{i}, g_{i+1}, \ldots, g_{n}\right)^{(-1)^{i-1}}\right. \\
\quad & \left.\xi \xi\left(\mathrm{r}(g) ; g, g_{1}, \ldots, g_{n-1}\right)^{(-1)^{n}}\right) .
\end{aligned}
$$




\section{Setting}

$\eta\left(g ; g_{1}, \ldots, g_{n-1}\right)$

$$
=\alpha_{g}^{-1}\left(\xi\left(\mathrm{r}(g) ; g, g_{1}, \ldots, g_{n-1}\right)\right) \in A(\mathrm{~s}(g)) ; \quad \eta \in \mathrm{C}_{\beta}^{n-1}(\mathscr{G}, \mathscr{B}),
$$

we compute for $\left(g, g_{1}, \ldots, g_{n}\right) \in \mathscr{G}^{(n+1)}$ with $y=\mathrm{r}(g)$

$$
\begin{aligned}
& (\partial \eta)\left(g ; g_{1}, \ldots, g_{n}\right) \\
& =\alpha_{g_{1}}\left(\eta\left(g g_{1} ; g_{2}, \ldots, g_{n}\right)\right) \\
& \quad \times \prod_{i=1}^{n-1} \eta\left(g ; g_{1}, \ldots, g_{i-1}, g_{i} g_{i+1}, g_{i+2}, \ldots, g_{n}\right)^{(-1)^{i}} \\
& \quad \times \eta\left(g ; g_{1}, g_{2}, \ldots, g_{n-1}\right)^{(-1)^{n}} \\
& =\alpha_{g_{1}}\left(\alpha_{g g_{1}}^{-1}\left(\xi\left(y ; g g_{1}, g_{2}, \ldots, g_{n}\right)\right)\right) \\
& \quad \times \prod_{i=1}^{n-1} \alpha_{g}^{-1}\left(\xi\left(y ; g, g_{1}, \ldots, g_{i-1}, g_{i} g_{i+1}, g_{i+2}, \ldots, g_{n}\right)\right)^{(-1)^{i}} \\
& \quad \times \alpha_{g}^{-1}\left(\xi\left(y ; g, g_{1}, g_{2}, \ldots, g_{n-1}\right)\right)^{(-1)^{n}} \\
& =\xi\left(g ; g_{1}, \ldots, g_{n}\right)
\end{aligned}
$$

This completes the proof.

Each $A(x)$ is a submodule of $B(x)$ for each $x \in X$, hence we get an exact sequence:

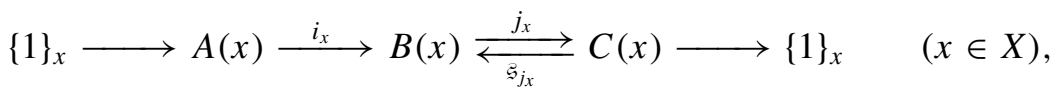

and another $\mathscr{G}$-module $\mathscr{C}$ :

$$
\mathscr{C}=\bigcup_{x \in X}^{\bullet} C(x), \quad \gamma_{g}(b A(x)) \stackrel{\text { def }}{=} \beta_{g}(b) A(y), \quad b \in B(x), \quad g=(y, g, x) \in \mathscr{G} .
$$

Symbolically we can write the exact sequence of $\mathscr{G}$-modules:

$$
X \longrightarrow \mathscr{A} \stackrel{i}{\longrightarrow} \mathscr{B} \stackrel{j}{\longrightarrow} \mathscr{C} \longrightarrow X .
$$

Take $\xi \in \mathrm{Z}_{\alpha}^{n}(\mathscr{G}, \mathscr{A})$. With

$$
\begin{aligned}
& \left(i_{*} \xi\right)\left(g ; g_{1}, \ldots, g_{n}\right) \\
& \quad=\xi\left(g_{1}, \ldots, g_{n}\right) \in A\left(\mathrm{r}\left(g_{1}\right)\right), \quad\left(g, g_{1}, \ldots, g_{n}\right) \in \mathscr{G}^{(n+1)},
\end{aligned}
$$


we obtain a cocycle $i_{*} \xi \in \mathrm{Z}_{\beta}^{n}(\mathscr{G}, \mathscr{B})=\mathrm{B}_{\beta}^{n}(\mathscr{G}, \mathscr{B})$ by Proposition 1.1 . Thus there exists a cochain $\eta \in \mathrm{C}_{\beta}^{n-1}(\mathscr{G}, \mathscr{B})$ such that

$$
i_{*} \xi=\partial \eta
$$

Then set

$$
\begin{aligned}
\zeta\left(x ; g_{1}, \ldots, g_{n-1}\right) & =j_{x}\left(\eta\left(x ; g_{1}, \ldots, g_{n-1}\right)\right) \\
& =\eta\left(x ; g_{1}, \ldots, g_{n-1}\right) A(x) \in C(x) .
\end{aligned}
$$

Since $\partial j=j \partial$, we get $\zeta \in \mathrm{Z}_{\gamma}^{n-1}(\mathscr{G}, \mathscr{C})$ and naturally

$$
\mathrm{H}_{\alpha}^{n}(\mathscr{G}, \mathscr{A}) \cong \mathrm{H}_{\gamma}^{n-1}(\mathscr{G}, \mathscr{C})
$$

under the map: $[\xi] \in \mathrm{H}_{\alpha}^{n}(\mathscr{G}, \mathscr{A}) \mapsto[\zeta] \in \mathrm{H}_{\gamma}^{n-1}(\mathscr{G}, \mathscr{C})$. Summarizing the above discussion, we obtain:

Proposition 1.2 (Dimension Shifting). If $\{\mathscr{A}, \alpha\}$ is a $\mathscr{G}$-module, then there exists a natural $\mathscr{G}$-module $\{\mathscr{C}, \gamma\}$ and a natural isomorphism:

$$
\mathrm{H}_{\alpha}^{n}(\mathscr{G}, \mathscr{A}) \cong \mathrm{H}_{\gamma}^{n-1}(\mathscr{G}, \mathscr{C})
$$

\section{Pullback, Reduction and Induction}

Let $\mathscr{H}$ be a groupoid with $Y=\mathscr{H}^{(0)}$. Suppose that $f$ is a surjective map from a space $X$ onto $Y$ (if applicable, we assume that the map $f$ is Borel).

Then we have a fibration of $X$ :

$$
X=\bigcup_{y \in Y}^{\bullet} X(y), X(y)=f^{-1}(y), y \in Y .
$$

Then we set

$$
\mathscr{G}=\bigcup_{(y, z) \in Y \times Y}\left\{X(z) \times \mathscr{H}_{z}^{y} \times X(y)\right\},
$$

where $\mathscr{H}_{y}^{z}=\mathrm{r}^{-1}(z) \cap \mathrm{s}^{-1}(y),(z, y) \in Y^{2}$. We then define the range and the source maps and

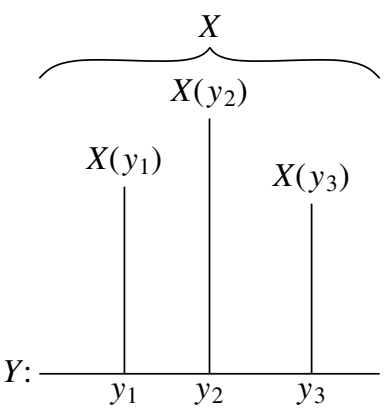
the product in $\mathscr{G}$ as follows:

$$
\begin{gathered}
\mathrm{r}(z, h, x)=z, \quad \mathrm{~s}(z, h, x)=x ; \\
(z, g, y)(y, h, x)=(z, g h, x), \quad(g, h) \in \mathscr{H}^{(2)}, \\
z \in X(\mathrm{r}(g)), y \in X(\mathrm{~s}(g))=X(\mathrm{r}(h)), x \in X(\mathrm{~s}(h)) .
\end{gathered}
$$


Definition 1.3. The groupoid $\mathscr{G}$ is called the pullback of $\mathscr{H}$ by the map $f$ and denoted by $f^{*}(\mathscr{H})$.

The map:

$$
f_{*}:(z, h, x) \in \mathscr{G} \mapsto f_{*}(z, h, x)=(f(z), h, f(x)) \in \mathscr{H}
$$

is a groupoid homomorphism of $\mathscr{G}$ onto $\mathscr{H}$.

If $f_{i}, i=1,2$, are maps from $X_{i}$ onto $Y$, then we have the fiber product $X=X_{1} * X_{2}$ relative to $f_{1}$ and $f_{2}$ :

$$
X=\left\{\left(x_{1}, x_{2}\right) \in X_{1} \times X_{2}: f_{1}\left(x_{1}\right)=f_{2}\left(x_{2}\right)\right\},
$$

and the map $f: x=\left(x_{1}, x_{2}\right) \in X \mapsto f(x)=f_{1}\left(x_{1}\right)=f_{2}\left(x_{2}\right) \in Y$ which makes the following diagram commutative:

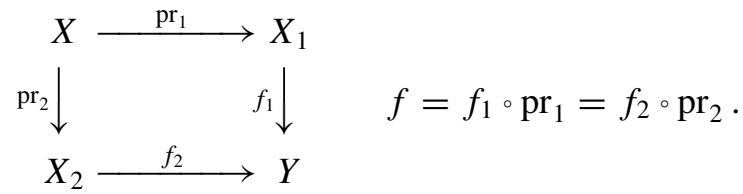

The pullbacks $\mathscr{G}=f^{*}(\mathscr{H}), \mathscr{G}_{1}=f_{1}^{*}(\mathscr{H}), \mathscr{G}_{2}=f_{2}^{*}(\mathscr{H})$ and $\mathscr{H}$ form the commutative diagram:

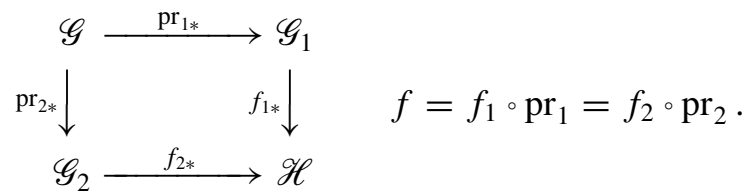

Let $Y \subset X$ be a subset such that the saturation $[Y]=X$, i.e.,

$$
[Y]=\mathrm{s} \circ \mathrm{r}^{-1}(Y)=X,
$$

equivalently for every $x \in X$ there exists $g=(y, g, x) \in \mathscr{G}$ such that $y \in Y$. If applicable, we assume that the set $Y$ is a Borel subset of $X$.

Definition 1.4. In the above setting, let $\mathscr{G}_{Y}$ be the set of all those $g \in \mathscr{G}$ such that $\mathrm{r}(g) \in Y$ and $\mathrm{s}(g) \in Y$, i.e.,

$$
\mathscr{G}_{Y}=\mathrm{r}^{-1}(Y) \cap \mathrm{s}^{-1}(Y) .
$$

Proposition 1.5. If $Y$ is a subset of $X$ such that $X=[Y]$, then there exists a surjective map $f: X \mapsto Y$ such that $\mathscr{G}$ is naturally isomorphic to the pullback groupoid $f^{*}\left(\mathscr{G}_{Y}\right)$. 
Proof. Consider the map

$$
\mathrm{s}: g \in \mathrm{r}^{-1}(Y) \mapsto x=\mathrm{s}(g) \in X
$$

and its cross-section (if applicable, we take a Borel cross-section $\gamma$ )

$$
\gamma: x \in X \mapsto \gamma(x) \in \mathrm{r}^{-1}(Y),
$$

such that $\gamma(y)=y$ if $y \in Y$. Set

$$
f(x)=\mathrm{r}(\gamma(x)) \in Y, \quad x \in X .
$$

We claim $\mathscr{G} \cong f^{*}\left(\mathscr{G}_{Y}\right)$. For each $(z, g, x) \in f^{*}\left(\mathscr{G}_{Y}\right)$, set

$$
\pi(z, g, x)=\gamma(z)^{-1} g \gamma(x) \in \mathscr{G}
$$

which makes sense because

$$
\begin{aligned}
& \mathrm{s}\left(\gamma(z)^{-1}\right)=\mathrm{r}(\gamma(z))=f(z)=\mathrm{r}(g) \in Y ; \\
& \mathrm{r}(\gamma(x))=f(x)=\mathrm{s}(g) .
\end{aligned}
$$

For each $((z, g, y),(y, h, x)) \in f^{*}\left(\mathscr{G}_{Y}\right)^{(2)}$, we get

$$
\begin{aligned}
\pi((z, g, y)(y, h, x)) & =\pi(z, g h, x)=\gamma(z)^{-1} g h \gamma(x) \\
& =\gamma(z)^{-1} g \gamma(y) \gamma(y)^{-1} h \gamma(x) \\
& =\pi(z, g, y) \pi(y, h, x) .
\end{aligned}
$$

Hence the map $\pi$ is multiplicative. The inverse $\pi^{-1}$ is given by:

$$
\pi^{-1}(z, g, x)=\left(z, \gamma(z) g \gamma(x)^{-1}, x\right), \quad(z, g, x) \in \mathscr{G} \text {. }
$$

This proves the assertion.

Similarly, if $\mathscr{B}$ is an $\mathscr{H}$-module, (not necessarily commutative), then the surjective map $f: X \mapsto Y$ also gives rise to the pullback $f^{*}(\mathscr{H})$-module $\mathscr{A}=f^{*}(\mathscr{B})$ in the following way:

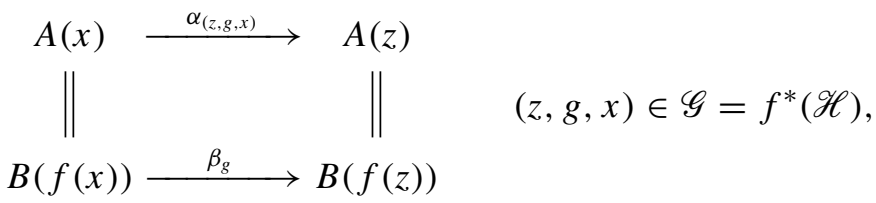

where $\beta$ is the action of $\mathscr{H}$ on $\mathscr{B}$. 
Definition 1.6. i) If $\mathscr{H}=\mathscr{G}_{Y}, Y \subset X$, and the map $f$ is given by Proposition 1.5, then the above $\mathscr{G}$-module $f^{*}(\mathscr{B})$ is called the induced $\mathscr{G}$ module and written $\mathscr{A}=\operatorname{Ind}_{Y}^{X} \mathscr{B}$ or $\mathscr{A}=\operatorname{Ind}_{Y \uparrow X} \mathscr{B}$.

ii) If $\mathscr{A}$ is a $\mathscr{G}$-module, then

$$
\mathscr{A}_{Y}=\bigcup_{y \in Y} A(y)
$$

is naturally $\mathscr{G}_{Y}$-module, which will be called the reduced module over $\mathscr{G}_{Y}$ or the reduced $\mathscr{G}_{Y}$-module.

Proposition 1.7. If $X=[Y]$, then every $\mathscr{G}_{\text {-module }} \mathscr{A}$, not necessarily commutative, is obtained from the reduced $\mathscr{G}_{Y}$-module $\mathscr{A}_{Y}$ as the induced module.

The proof is exactly the same as Proposition 1.5, and we leave details to the interested reader.

Proposition 1.8. If $Y \subset X$ and $X=[Y]$, then the embedding map

$$
i_{Y}: \mathscr{G}_{Y} \hookrightarrow \mathscr{G}
$$

gives rise to the pullback map, i.e., the restriction map, with the following properties:

$$
\begin{gathered}
i_{Y}^{*}: \xi \in \mathrm{Z}_{\alpha}^{n}(\mathscr{G}, \mathscr{A}) \mapsto \xi_{Y}=\left.\xi\right|_{Y} \in \mathrm{Z}_{\alpha}^{n}\left(\mathscr{G}_{Y}, \mathscr{A}_{Y}\right) ; \\
f_{*}^{*} i_{Y}^{*}(\xi) \equiv \xi \bmod \mathrm{B}_{\alpha}^{n}(\mathscr{G}, \mathscr{A}), \quad \xi \in \mathrm{Z}_{\alpha}^{n}(\mathscr{G}, \mathscr{A}) ; \\
i_{Y}^{*} \circ f_{*}^{*}(\xi) \equiv \xi \quad \bmod \mathrm{B}_{\alpha}^{n}\left(\mathscr{G}_{Y}, \mathscr{A}_{Y}\right), \quad \xi \in \mathrm{Z}_{\alpha}^{n}\left(\mathscr{G}_{Y}, \mathscr{A}_{Y}\right) ; \\
i_{Y}^{*}: \mathrm{H}_{\alpha}^{n}(\mathscr{G}, \mathscr{A}) \cong \mathrm{H}_{\alpha}^{n}\left(\mathscr{G}_{Y}, \mathscr{A}_{Y}\right),
\end{gathered}
$$

where $f$ is the map of Proposition 1.5 and the map $f_{*}: \mathscr{G} \longmapsto \mathscr{G}_{Y}$ is given by $f_{*}(g)=\gamma(z) g \gamma(x)^{-1}, g=(z, g, x) \in \mathscr{G}$.

Proof. In view of the last two propositions, we may and do assume that the groupoid $\mathscr{G}$ and the $\mathscr{G}$-module $\mathscr{A}$ are both obtained as $\mathscr{G}=f^{*}\left(\mathscr{G}_{Y}\right)$ and $\mathscr{A}=f^{*}\left(\mathscr{A}_{Y}\right)$. In this setting, we get $A(x)=A(f(x))$ and $\alpha_{\gamma(x)}=\operatorname{id}_{A(x)}$, the identity map on $A(x)$, for every $x \in X$.

For $n=0$, each $\xi \in Z_{\alpha}^{0}(\mathscr{G}, \mathscr{A})$ is a map $\xi: x \in X \mapsto \xi(x) \in A(x)$ such that

$$
\xi(\mathrm{r}(g))=\alpha_{g}(\xi(\mathrm{s}(g))), \quad g \in \mathscr{G} .
$$

The restriction $\xi_{Y}$ satisfies the same identities for $\mathscr{G}_{Y}$ and $\xi(x)=\xi_{Y}(f(x)), x \in$ $X$. Hence $\xi_{Y} \in Z_{\alpha}^{0}\left(\mathscr{G}_{Y}, \mathscr{A}_{Y}\right)$. 
If $\eta \in \mathrm{Z}_{\alpha}^{0}\left(\mathscr{G}_{Y}, \mathscr{A}_{Y}\right)$, then $\xi=f_{*}^{*} \eta$ satisfies, for each $g=\left(z, f_{*}(g), x\right) \in \mathscr{G}$,

$$
g=\gamma(z)^{-1} f_{*}(g) \gamma(x),
$$

and

$$
\xi(z)=\eta\left(\mathrm{r}\left(f_{*}(g)\right)\right)=\alpha_{f_{*}(g)}(\eta(f(x)))=\alpha_{f_{*}(g)}(\xi(x))=\alpha_{g}(\xi(x)) .
$$

Hence we get $\xi=f_{*}^{*} \eta \in \mathrm{Z}_{\alpha}^{0}(\mathscr{G}, \mathscr{A})$.

Since $\xi(x)=\xi(f(x)), x \in X$, for every $\xi \in Z_{\alpha}^{0}(\mathscr{G}, \mathscr{A})$, we conclude that $\mathrm{H}_{\alpha}^{0}(\mathscr{G}, \mathscr{A}) \cong \mathrm{H}_{\alpha}^{0}\left(\mathscr{G}_{Y}, \mathscr{A}_{Y}\right)$ under the isomorphism $i_{Y}^{*}$.

The case $n=1$ : Each $\xi \in \mathrm{Z}_{\alpha}^{1}(\mathscr{G}, \mathscr{A})$ satisfies

$$
\xi(g h)=\xi(g) \alpha_{g}(\xi(h)), \quad(g, h) \in \mathscr{G}^{(2)} .
$$

The restriction $\xi_{Y}$ satisfies the same identity, so that it is a cocycle in $Z_{\alpha}^{1}\left(\mathscr{G}_{Y}, \mathscr{A}_{Y}\right)$. Now choose $\xi_{Y} \in \mathrm{Z}_{\alpha}^{1}\left(\mathscr{G}_{Y}, \mathscr{A}_{Y}\right)$ and set

$$
\xi(z, g, x)=\left(f_{*}^{*} \xi_{Y}\right)(z, g, x)=\xi_{Y}\left(\gamma(z) g \gamma(x)^{-1}\right) .
$$

For each pair $g=(z, g, y), h=(y, h, x) \in \mathscr{G}$, we have

$$
\begin{aligned}
\xi(g h) & =\xi_{Y}\left(f_{*}(g h)\right)=\xi_{Y}\left(f_{*}(g) f_{*}(h)\right) \\
& =\xi_{Y}\left(f_{*}(g)\right) \beta_{f_{*}(g)}\left(\xi_{Y}\left(f_{*}(h)\right)=\xi(g) \alpha_{g}(\xi(h)) .\right.
\end{aligned}
$$

Hence $f_{*}^{*}\left(\xi_{Y}\right) \in Z_{\alpha}^{1}(\mathscr{G}, \mathscr{A})$.

Suppose $\xi_{Y}=i_{Y}^{*}(\xi)$. We then compare $\xi$ and $f_{*}^{*} \xi_{Y}$. For $g=(z, g, x)$, we write $f_{*}(g)=\left(f(z), f_{*}(g), f(x)\right)=\gamma(z) g \gamma(x)^{-1} \in \mathscr{G}_{Y}$ and compute:

$$
\begin{aligned}
\left(f_{*}^{*} \xi\right)(g) & =\xi\left(\gamma(z) g \gamma(x)^{-1}\right)=\xi(\gamma(z)) \xi\left(g \gamma(x)^{-1}\right) \quad\left(\text { as } \alpha_{\gamma(z)}=\mathrm{id}\right) \\
& =\xi(\gamma(z)) \xi(g) \alpha_{g}\left(\xi\left(\gamma(x)^{-1}\right)\right) \\
& =\xi(\gamma(z)) \xi(g) \alpha_{g}\left(\alpha_{\gamma(x)}^{-1}\left(\xi(\gamma(x))^{-1}\right)\right) \\
& =\xi(\gamma(z)) \xi(g) \alpha_{g}\left(\xi(\gamma(x))^{-1}\right) .
\end{aligned}
$$

Hence the above calculation becomes:

$$
\left(f_{*}^{*} \xi\right)(g)=\xi(\gamma(z)) \xi(g) \alpha_{g}\left(\xi(\gamma(x))^{-1}\right) .
$$

Therefore we get $\xi \equiv f_{*}^{*} \xi \bmod _{\alpha}^{1}(\mathscr{G}, \mathscr{A})$.

For $n=2,3, \ldots$, we use the dimension shifting, Proposition 1.2. From the construction of $\{\mathscr{C}, \gamma\}$ from $\{\mathscr{A}, \alpha\}$, it follows that the reduced $\mathscr{G}_{Y}$-modules 
$\mathscr{C}_{Y}$ and $\mathscr{A}_{Y}$ are related in the exactly same way as the original modules $\mathscr{A}$ and $\mathscr{C}$ are. Hence we have by mathematical induction:

$$
\mathrm{H}_{\alpha}^{n}(\mathscr{G}, \mathscr{A}) \cong \mathrm{H}_{\gamma}^{n-1}(\mathscr{G}, \mathscr{C}) \cong \mathrm{H}_{\gamma}^{n-1}\left(\mathscr{G}_{Y}, \mathscr{C}_{Y}\right) \cong \mathrm{H}_{\alpha}^{n}\left(\mathscr{G}_{Y}, \mathscr{A}_{Y}\right)
$$

Tracing the isomorphims, we conclude that the isomorphism is indeed given by $f_{*}^{*}$ and $i_{Y}^{*}$.

REMARK 1.9. In the case that $Y$ is a singleton set $\left\{y_{0}\right\}$, the reduced groupoid $\mathscr{G}_{Y}$ is a group, say $H$. The associated principal groupoid $\widetilde{\mathscr{G}}$, the equivalence relation groupoid given by the orbit structure of $\mathscr{G}$, is transitive. This is precisely the conventional induction procedure and also the Shapiro mechanism. This case is also relevant to us in the case of a system based on a factor of type $\mathrm{III}_{\lambda}$ as will be seen in the later sections.

Definition 1.10. A normal subgroupoid $\mathcal{N}$ of $\mathscr{G}$ is a field

$$
\left\{N(x) \subset \mathrm{r}^{-1}(x) \cap \mathrm{s}^{-1}(x): x \in X\right\}
$$

of groups such that

$$
N(y)=g N(x) g^{-1}, \quad(y, g, x) \in \mathscr{G} .
$$

For a commutative $\mathscr{G}$-module $\mathscr{A}$ with trivial action of $\mathcal{N}$, we can define the group $\mathrm{Z}_{\alpha}(\mathscr{G}, \mathcal{N}, \mathscr{A})$ of characteristic cocycles $(\lambda, \mu)$ as in [24] and the group $\Lambda_{\alpha}(\mathscr{G}, \mathscr{N}, \mathscr{A})$ of characteristic invariants. Each $(\lambda, \mu) \in \mathrm{Z}_{\alpha}(\mathscr{G}, \mathcal{N}, \mathscr{A})$ gives rise to an exact sequence of $\mathscr{G}$-modules equipped with cross-section $\mathfrak{G}$ :

$$
\mathscr{E}: X \longrightarrow \mathscr{A} \stackrel{i}{\longrightarrow} \mathscr{E}=\mathscr{A} \rtimes_{\alpha, \mu} \mathcal{N} \underset{\xi}{\stackrel{j}{\rightleftarrows}} \mathcal{N} \longrightarrow X
$$

where

$$
\begin{gathered}
\mathfrak{g}:(m, x) \in N(x) \mapsto \mathfrak{S}(m, x)=\left(1_{x}, m\right) \in \mathscr{E}(x)=A(x) \rtimes_{\alpha, \mu_{x}} N(x) ; \\
\mathfrak{S}(m, x) \mathfrak{S}(n, x)=\mu(m, n ; x) \mathfrak{S}(m n, x)=\mu_{x}(m, n) \mathfrak{S}(m n, x) ; \\
\alpha_{z, g, x}\left(\mathfrak{S}\left(g^{-1} m g, x\right)\right)=\lambda(m ; z, g, x) \mathfrak{S}(m, z), \quad(z, g, x) \in \mathscr{G},(m, x) \in N(x) .
\end{gathered}
$$

Conversely, if we have an exact sequence of $\mathscr{G}$-modules:

$$
\mathscr{E}: X \longrightarrow \mathscr{A} \stackrel{i}{\longrightarrow} \mathscr{E} \stackrel{j}{\longrightarrow} \mathcal{N} \longrightarrow X
$$

then a cross-section $\mathfrak{S}$ (if applicable, we take a Borel cross-section) of the map $j$ gives rise to $\mathscr{A}$-valued functions $\lambda$ on $\mathcal{N} *_{\mathrm{r}} \mathscr{G}=\{(m, g) \in \mathcal{N} \times \mathscr{G}: \mathrm{s}(m)=$ 
$\mathrm{r}(g)\}$ and $\mu$ on $\mathscr{N}^{(2)}$ such that

$$
\begin{aligned}
\mathfrak{S}(m, x) \mathfrak{S}(n, x) & =\mu(m, n ; x) \mathfrak{S}(m n, x), \quad(m, x),(n, x) \in N(x) ; \\
\alpha_{z, g, x}\left(\mathfrak{S}\left(g^{-1} m g, x\right)\right) & =\lambda(m ; z, g, x) \mathfrak{S}(m, z),((m, x),(z, g, x)) \in \mathcal{N}_{x} \times \mathscr{G}_{x}^{z},
\end{aligned}
$$

and the pair $(\lambda, \mu)$ falls in the group $\mathrm{Z}_{\alpha}(\mathscr{G}, \mathcal{N}, \mathscr{A})$. We denote the collection of the conjugate classes of exact sequences by $\operatorname{Xext}(\mathscr{G}, \mathscr{N}, \mathscr{A})$ and each exact sequence a crossed extension of $\mathscr{A}$ by $\mathscr{N}$. The group multiplication in $\mathrm{Z}_{\alpha}(\mathscr{G}, \mathcal{N}, \mathscr{A})$ reflects to the following operations in $\operatorname{Xext}(\mathscr{G}, \mathcal{N}, \mathscr{A})$ :

i) For any two crossed extensions $\mathscr{E}_{1}, \mathscr{E}_{2} \in \operatorname{Xext}(\mathscr{G}, \mathcal{N}, \mathscr{A})$ :

$$
\begin{aligned}
& \mathscr{E}_{1}: X \longrightarrow \mathscr{A} \stackrel{i_{1}}{\longrightarrow} \mathscr{E}_{1} \stackrel{j_{1}}{\longrightarrow} \mathcal{N} \longrightarrow X ; \\
& \mathscr{E}_{2}: X \longrightarrow \mathscr{A} \stackrel{i_{2}}{\longrightarrow} \mathscr{E}_{2} \stackrel{j_{2}}{\longrightarrow} \mathcal{N} \longrightarrow X
\end{aligned}
$$

the product crossed extension $\mathscr{E}$ is defined to be the quotient module of the fiber product:

$$
\begin{aligned}
\mathscr{E} & =\mathscr{E}_{1} * \mathscr{E}_{2} \\
& =\left\{\left(e_{1}, e_{2}\right) \in \mathscr{E}_{1} \times \mathscr{E}_{2}: j_{1}\left(e_{1}\right)=j_{2}\left(e_{2}\right)\right\} /\left\{\left(i_{1}(a), i_{2}\left(a^{-1}\right)\right): a \in \mathscr{A}\right\} .
\end{aligned}
$$

ii) The inverse $\mathscr{E}^{-1}$ is then given by:

$$
\mathscr{E}^{\prime}: X \longrightarrow \mathscr{A} \stackrel{\left\{a \in \mathscr{A} \mapsto i\left(a^{-1}\right)\right\}}{\longrightarrow} \mathscr{E} \longrightarrow \mathcal{N} \longrightarrow X
$$

Proposition 1.11. If $Y \subset X$ is a subset of $X$ such that $X=[Y]=$ $\mathrm{s}\left(\mathrm{r}^{-1}(Y)\right)$, then with the map $f$ of Proposition 1.5 the maps $f_{*}^{*}$ and $i_{Y}^{*}$ give isomorphisms between $\Lambda_{\alpha}(\mathscr{G}, \mathcal{N}, \mathscr{A})$ and $\Lambda_{\alpha}\left(\mathscr{G}_{Y}, \mathscr{N}_{Y}, \mathscr{A}_{Y}\right)$ which are the inverse of one another.

Proof. First, we may and do assume that $\mathscr{A}=\operatorname{Ind}_{Y}^{X} \mathscr{B}$ and $\mathscr{G}=f^{*}(\mathscr{H})$ with $\mathscr{B}=\mathscr{A}_{Y}$ and $\mathscr{H}=\mathscr{G}_{Y}$. Also the normal subgroupoid $\mathscr{N}$ is a $\mathscr{G}$-module and therefore it is conjugate to the induced $\mathscr{G}$-module $\operatorname{Int}_{Y}^{X}(\mathscr{M})$ with $\mathscr{M}=\mathscr{N}_{Y}$ as a $\mathscr{G}$-module. However, it is not entirely trivial to relate the structure of the inclusions: $\mathscr{M} \subset \mathscr{G}_{Y}$ and $\mathcal{N} \subset \mathscr{G}$. We have to study the way that the entire groupoid $\mathscr{G}$ is related to the reduced one $\mathscr{G}_{Y}$. As $\mathscr{G}=f^{*}\left(\mathscr{G}_{Y}\right)$, we have

$$
\begin{gathered}
\mathscr{G}_{x}^{z}=\{z\} \times \mathscr{H}_{f(x)}^{f(z)} \times\{x\} ; \\
\mathscr{G}_{x}^{x}=\{x\} \times \mathscr{H}_{f(x)}^{f(x)} \times\{x\}=\gamma(x)^{-1} \mathscr{H}_{f(x)}^{f(x)} \gamma(x) ; \\
N(x)=\gamma(x)^{-1} N(f(x)) \gamma(x)=\{x\} \times M(f(x)) \times\{x\} .
\end{gathered}
$$


Hence we have $\mathcal{N}=f^{*}(\mathcal{M})$.

Now every $\mathscr{E} \in \operatorname{Xext}_{\alpha}(\mathscr{G}, \mathcal{N}, \mathscr{A})$ conjugate to a crossed extension of the form $f^{*}(\mathscr{F})$ with $\mathscr{F} \in \operatorname{Xext}_{\alpha}(\mathscr{H}, \mathscr{M}, \mathscr{B})$ and $\mathscr{F}$ is given as $\mathscr{F}=\mathscr{E}_{Y}$. It is straightforward now to see that $f^{*}\left(\mathscr{F}_{1}\right) \cong f^{*}\left(\mathscr{F}_{2}\right)$ if and only if $\mathscr{F}_{1} \cong \mathscr{F}_{2}$. Hence the $f_{*}^{*}$ and $i_{Y}^{*}$ are isomorphisms between $\Lambda_{\alpha}(\mathscr{G}, \mathcal{N}, \mathscr{A})$ and $\Lambda_{\alpha}\left(\mathscr{G}_{Y}\right.$, $\mathcal{N}_{Y}, \mathscr{A}_{Y}$ ) which are inverse of one another.

\section{Non-Polish Groupoid}

In many cases, we encounter the following situation:

i) An ergodic flow $\{\mathcal{A}, \mathrm{R}, \theta\}$ is given on an abelian separable von Neumann algebra $\mathcal{A}$. Let $\{X, \mathrm{~m}\}$ be the measure theoretic spectrum of $\mathcal{A}$, i.e., $\{X, \mathrm{~m}\}$ is a standard measure space such that $\mathcal{A}=L^{\infty}(X, \mathrm{~m})$;

ii) A Polish group $H$ acts on the flow $\{\mathcal{A}, \mathrm{R}, \theta\}$ via $\alpha$, i.e., $\alpha$ is a homomorphism of $H$ into the group $\operatorname{Aut}_{\theta}(\mathcal{A})=\left\{\sigma \in \operatorname{Aut}(\mathcal{A}): \sigma \circ \theta_{s}=\theta_{s} \circ \sigma, s \in \mathrm{R}\right\}$, which gives rise to a joint action of $\widetilde{H}=H \times \mathrm{R}$ on $\mathcal{A}$. We denote it by $\alpha$ also and by $\theta$ if we restrict $\alpha$ to the action of R. We also use the notations:

$$
\left(\alpha_{g, s} f\right)(x)=f\left(T_{g, s}^{-1} x\right), \quad f \in \mathcal{A}=L^{\infty}(X, \mathrm{~m}),(g, s) \in \widetilde{H} .
$$

iii) A normal subgroup $L$ of $H$ contained in the $\operatorname{kernel} L \subset \operatorname{Ker}(\alpha)$ is given, so that the action of $H$ factors through the quotient group $Q=H / L$;

iv) The normal subgroup $L$ is not closed, so that the quotient group $Q$ does not have a reasonable topological or Borel structure despite its significance in the theory.

The action $\alpha$ of $\widetilde{H}$ on $\mathcal{A}$ gives rise to the action of $\widetilde{Q}=Q \times \mathrm{R}$ which will be denoted by $\alpha$ again. Now the groupoid $\mathscr{G}_{\widetilde{Q}}=X \rtimes \widetilde{Q}$ is the groupoid which is relevant to us despite the lack of a reasonable Borel structure on it. In what follows, we consider the discrete topology on $Q$ side and the usual topological and Borel structures on R-side. Namely we are going to consider those functions $f$ on $\mathscr{G}_{\widetilde{Q}}$ such that the map: $(x, s) \mapsto f(x, q, s)$ is jointly Borel as a function on $X \times \mathrm{R}$ for each fixed $q \in Q$. Namely, we consider the product Borel structure of those on $X$ and $\mathrm{R}$ and the discrete Borel structure on $Q$. A typical example will be the automorphism group $\operatorname{Aut}(\mathcal{M})$ of a separable factor $\mathcal{M}$ as $H$ and $\operatorname{Cnt}_{\mathrm{r}}(\mathcal{M})$ as $L$ and the flow $\{\mathcal{C}, \mathrm{R}, \theta\}$ of weights on $\mathcal{M}$ as $\{\mathcal{A}, \mathbf{R}, \theta\}$.

\section{Model Construction II}

Let $\{\mathcal{C}, \mathbf{R}, \theta\}$ be an ergodic flow to be fixed throughout this section. Let $H$ be a countable discrete group and $\alpha$ an action of $H$ on the flow $\{\mathcal{C}, \mathbf{R}, \theta\}$, i.e., 
a homomorphism of $H$ into the group $\operatorname{Aut}_{\theta}(\mathcal{C})$ of all automorphisms of $\mathcal{C}$ commuting with the flow $\theta$. Let $\{X, \mathrm{~m}\}$ be the measure spectrum of $\mathcal{C}$, i.e., a standard $\sigma$-finite measure space on which $\mathrm{R}$ acts as a one-parameter group of non-singular transformations $\left\{T_{s}: s \in \mathrm{R}\right\}$, so that

$$
\theta_{s} f(x)=f\left(T_{s}^{-1} x\right), \quad f \in \mathcal{C}=L^{\infty}(X, \mathrm{~m}), x \in X, s \in \mathrm{R} .
$$

We denote the action $\alpha$ of $H$ on the space $X$ in the following fashion:

$$
\alpha_{g} f(x)=f(x g), \quad g \in H, f \in \mathcal{C} .
$$

When we consider the joint action of $\widetilde{H}=H \times \mathrm{R}$ on $X$, denoted simply by $\alpha$ also, we write

$$
\left(\alpha_{\tilde{g}} f\right)(x)=f(x \tilde{g})=f\left(T_{s}^{-1} x g\right), \quad \tilde{g}=(g, s) \in \widetilde{H}=H \times \mathrm{R} .
$$

Let $L$ be a normal subgroup of $H$ contained in the $\operatorname{Kernel} \operatorname{Ker}(\alpha)$ of $\alpha$, i.e., $L$ does not act on $\mathcal{C}$ at all.

This section will be devoted to a construction of an action $\alpha$ of $H$ on a separable strongly stable factor $\mathcal{M}$ for any characteristic invariant $\chi \in$ $\Lambda_{\alpha}(\widetilde{H}, L, U(\mathcal{C}))$ such that

i) The flow of weights on $\mathcal{M}$ is conjugate to the flow $\{\mathcal{C}, R, \theta\}$, which will be identified;

ii) The modulus $\bmod (\alpha)$ is precisely the preassigned action $\alpha$ on $\mathcal{C}$ of $H$;

$$
\begin{gathered}
L=\alpha^{-1}\left(\operatorname{Cnt}_{\mathrm{r}}(\mathcal{M})\right) ; \\
\chi=\chi(\alpha) .
\end{gathered}
$$

Here the strong stability of $\mathcal{M}$ means that $\mathcal{M} \cong \mathcal{M} \bar{\otimes} \mathcal{R}_{0}$ with $\mathcal{R}_{0}$ an approximately finite dimensional factor of type $\mathrm{II}_{1}$. This is equivalent to the non commutativity of the quotient group $\overline{\operatorname{Int}}(\mathcal{M}) / \operatorname{Int}(\mathcal{M})$ of the group $\overline{\operatorname{Int}}(\mathcal{M})$ of approximately inner automorphisms by the $\operatorname{group} \operatorname{Int}(\mathcal{M})$ of inner automorphisms.

The joint action $\widetilde{H}$ on $X$ gives rise to a standard measured groupoid $\mathscr{H}=$ $X \times \widetilde{H}$ such that

$$
\begin{gathered}
\mathrm{r}(y, \tilde{g})=y \quad \mathrm{~s}(y, \tilde{g})=y \tilde{g}, \quad y \in X, \tilde{g}=(g, s) \in \widetilde{H}=H \times \mathrm{R} \\
(y, \tilde{g})(y \tilde{g}, \tilde{h})=(y, \tilde{g} \tilde{h}), \quad \tilde{h}=(h, t) \in \widetilde{H} .
\end{gathered}
$$

In order to shorten notations we write

$$
\tilde{g} \in \mathscr{G} \text { and } \tilde{h} \in \mathscr{G} \text { for }(y, \tilde{g}) \in \mathscr{G} \text { and }(x, \tilde{h}) \in \mathscr{G}
$$


respectively omitting the range $y=\mathrm{r}(\tilde{g}), x=\mathrm{r}(\tilde{h}) \in X$. At the same time, to specify the range and the source explicitly, we often write

$$
(y, \tilde{g}, x) \in \mathscr{G} \text { for } y=\mathrm{r}(y, \tilde{g}, x) \text { and } x=\mathrm{s}(y, \tilde{g}, x) \text {, i.e., } x=y \tilde{g} \text {. }
$$

For each $x \in X$, let $K(x)$ be the isotropy group of $x$, i.e.,

$$
K(x)=\{\tilde{g} \in \tilde{H}: x=x \tilde{g}\} .
$$

The map: $x \in X \mapsto K(x) \in \operatorname{Sub}(\widetilde{H})$ is a Borel map from the standard Borel space $X$ into the standard Borel space $\operatorname{Sub}(\widetilde{H})$ of all closed subgroups of $\widetilde{H}$ such that $K(x)=K\left(T_{s} x\right), s \in \mathrm{R}, x \in X$, since the flow $\theta$ and the joint action $\alpha$ of $\widetilde{H}$ commute. The ergodicity of $\theta$ then implies that $K(x)=K \in \operatorname{Sub}(\widetilde{H})$ for some fixed closed subgroup $K$ of $\widetilde{H}$. Then of course, $K$ is the Kernel $\operatorname{Ker}(\alpha)$ of the joint action $\alpha$ of $\widetilde{H}$, hence it is a normal closed subgroup of $\widetilde{H}$ which contains the normal subgroup $L$. Let $\mathcal{N}=X \times L$ denote the normal subgroupoid of $\mathscr{G}$.

Proposition 2.1. Let $A$ be the unitary group $\mathcal{U}(\mathcal{C})$ of $\mathcal{C}$. Then there is a natural isomorphism: $(\lambda, \mu) \in \mathrm{Z}_{\alpha}(\widetilde{H}, L, A) \mapsto(\tilde{\lambda}, \tilde{\mu}) \in \mathrm{Z}(\mathscr{H}, \mathcal{N}, \mathrm{T})$ from the group $\mathrm{Z}_{\alpha}(\tilde{H}, L, A)$ of characteristic cocycles onto the group $\mathrm{Z}(\mathscr{G}, \mathcal{N}, \mathrm{T})$ of characteristic cocycles which maps precisely the group $\mathrm{B}_{\alpha}(\widetilde{H}, L, A)$ of coboundaries onto the subgroup $\mathrm{B}(\mathscr{G}, \mathcal{N}, \mathrm{T})$ of coboundaries, so that it induces a natural isomorphism:

$$
\chi \in \Lambda_{\alpha}(\tilde{H}, L, A) \mapsto \tilde{\chi} \in \Lambda(\mathscr{G}, \mathcal{N}, \mathrm{T}) .
$$

Proof. To each $(\lambda, \mu) \in \mathrm{Z}_{\alpha}(\tilde{H}, L, A)$, we want to associate a cocycle $(\tilde{\lambda}, \tilde{\mu}) \in \mathrm{Z}(\mathscr{G}, \mathcal{N}, \mathrm{T})$. First, we realize $(\lambda(m, \tilde{g}), \mu(m, n))$ for $m, n \in L$ and $\tilde{g}=(g, s) \in \widetilde{H}$ as T-valued Borel functions over $X$ so that the cocycle identities hold almost everywhere and write them:

$$
\lambda(x ; m ; \tilde{g}) \text { and } \mu(x ; m, n), \quad x \in X, m, n \in L, \text { and } \tilde{g}=(g, s) \in \tilde{H} \text {. }
$$

Then set

$$
\begin{array}{lll}
\tilde{\mu}((x, m) ;(x, n))=\mu(x ; m, n), & ((x, m) ;(x, n)) \in \mathcal{N}^{(2)} ; \\
\tilde{\lambda}((x, m) ;(x, \tilde{g}))=\lambda(x ; m ; \tilde{g}), & ((x, m) ;(x, \tilde{g})) \in \mathscr{G}^{(2)} .
\end{array}
$$

Let us check the cocycle identity:

i) The 2-cocycle identity for $\mu$ guarantees the 2-cocycle identity for $\tilde{\mu}$ almost everywhere; 
ii) If $m=(z, m, z) \in \mathcal{N}$ and $(\tilde{g}=(z, \tilde{g}, y), \tilde{h}=(y, \tilde{h}, x)) \in \mathscr{G}^{(2)}$, then $y=z \tilde{g}, x=y \tilde{h}$ and

$$
\begin{aligned}
\tilde{\lambda}(m ; \tilde{g} \tilde{h}) & =\tilde{\lambda}((z, m, z) ;(z, \tilde{g} \tilde{h}, x))=\lambda(z ; m ; \tilde{g} \tilde{h}) \\
& =\lambda(z ; m ; \tilde{g}) \lambda\left(z \tilde{g} ; \tilde{g}^{-1} m \tilde{g} ; \tilde{h}\right) \\
& =\tilde{\lambda}((z, m, z) ;(z, \tilde{g}, z \tilde{g})) \tilde{\lambda}\left(\left(z \tilde{g}, \tilde{g}^{-1} m \tilde{g}, z \tilde{g}\right) ;(z \tilde{g}, \tilde{h}, x)\right) \\
& =\tilde{\lambda}((z, m, z) ;(z, \tilde{g}, y)) \tilde{\lambda}\left(\left(y, \tilde{g}^{-1} m \tilde{g}, y\right) ;(y, \tilde{h}, x)\right) \\
& =\tilde{\lambda}(m ; \tilde{g}) \lambda\left(\tilde{g}^{-1} m \tilde{g} ; \tilde{h}\right) .
\end{aligned}
$$

iii) For $m=(x, m, x), n=(x, n, x) \in \mathcal{N}$, we have

$$
\begin{aligned}
\tilde{\lambda}(m, n) & =\lambda(x ; m, n)=\mu\left(x ; n, n^{-1} m n\right) \mu^{*}(x ; m, n) \\
& =\tilde{\mu}\left(n, n^{-1} m n\right) \overline{\tilde{\mu}(m, n)} .
\end{aligned}
$$

iv) For each $m=(y, m, y) \in \mathcal{N}, \tilde{g}=(y, \tilde{g}, x) \in \mathscr{G}$, we have

$$
\begin{aligned}
\tilde{\lambda}(m ; \tilde{g}) \tilde{\lambda}(n ; \tilde{g}) \overline{\tilde{\lambda}(m n ; \tilde{g})} & =\lambda(y ; m ; \tilde{g}) \lambda(y ; n, \tilde{g}) \overline{\lambda(y ; m n ; \tilde{g})} \\
& =\mu\left(y \tilde{g} ; g^{-1} m g ; g^{-1} n g\right) \overline{\mu(y ; m, n)} \\
& =\tilde{\mu}\left(\tilde{g}^{-1} m g ; \tilde{g}^{-1} n \tilde{g}\right) \tilde{\tilde{\mu}(m, n)} .
\end{aligned}
$$

Therefore the pair $(\tilde{\lambda}, \tilde{\mu})$ is a characteristic cocycle for $\{\mathscr{G}, \mathcal{N}\}$ with values in T. The map: $(\lambda, \mu) \in \mathrm{Z}_{\alpha}(\widetilde{H}, L, A) \mapsto(\tilde{\lambda}, \tilde{\mu}) \in \mathrm{Z}(\mathscr{G}, \mathcal{N}, \mathrm{T})$ is an injective homomorphism.

Conversely, it is clear that if $(\tilde{\lambda}, \tilde{\mu}) \in \mathrm{Z}(\mathscr{G}, \mathcal{N}, \mathrm{T})$ is given, then the pair $(\lambda, \mu)$ defined by:

$$
\begin{aligned}
& \lambda(x ; m ; \tilde{g})=\tilde{\lambda}((x, m, x) ;(x, \tilde{g}, x \tilde{g})) \\
& \mu(x ; m, n)=\tilde{\mu}((x, m, x) ;(x, n, x))
\end{aligned} \quad \text { for } \quad x \in X, \tilde{g} \in \tilde{H}, m, n \in L,
$$

is an element of $\mathrm{Z}_{\alpha}(\tilde{H}, L, A)$.

Finally, each cochain $c: m \in L \mapsto c(m) \in A$ gives rise to a T-valued cochain:

$$
\tilde{c}(x, m, x)=c(x ; m) \in \mathrm{T}
$$

The correspondences $c \mapsto \tilde{c}$ and $(\lambda, \mu) \mapsto(\tilde{\lambda}, \tilde{\mu})$ intertwines the respective coboundary operations. Accordingly, $\mathrm{B}_{\alpha}(\widetilde{H}, L, A)$ corresponds exactly to $\mathrm{B}(\mathscr{G}, \mathcal{N}, \mathrm{T})$. This completes the proof.

Lemma 2.2. There exists a model construction:

$$
(\lambda, \mu) \in \mathrm{Z}_{\alpha}(\tilde{H}, L, A) \mapsto\left\{\mathcal{M}_{0}(\lambda, \mu), \widetilde{H}, \beta^{\lambda, \mu}\right\}
$$


such that

i) $\mathcal{M}_{0}(\lambda, \mu)$ is a separable von Neumann algebra of type $\mathrm{II}_{1}$;

ii) The restriction of the action $\beta^{\lambda, \mu}$ of $\widetilde{\tilde{H}}$ to the center of $\mathcal{M}_{0}(\lambda, \mu)$ is conjugate to the covariant system $\{\mathcal{C}, \widetilde{H}, \alpha\}$;

iii) $L=\left(\beta^{\lambda, \mu}\right)^{-1}(\operatorname{Int}(\mathcal{M}(\lambda, \mu)))$;

iv) There exists a map $u_{0}: m \in L \mapsto u_{0}(m) \in \mathcal{U}(\mathcal{M})$ such that

$$
\begin{aligned}
u_{0}(m) u_{0}(n) & =\mu(m, n) u_{0}(m n), \quad m, n \in L ; \\
\beta_{\tilde{g}}\left(u_{0}\left(g^{-1} m g\right)\right) & =\lambda(m ; \tilde{g}) u_{0}(m), \quad \tilde{g}=(g, s) \in \tilde{H}=H \times \mathbf{R} .
\end{aligned}
$$

If the original group $H$ is amenable, then the construction yields that the factor $\mathcal{M}_{0}(\lambda, \mu)$ is necessarily AFD.

Proof. First, we have the corresponding characteristic cocycle $(\tilde{\lambda}, \tilde{\mu}) \in$ $\mathrm{Z}_{\alpha}(\mathscr{G}, \mathcal{N}, \mathrm{T})$. Let $\mathcal{R}_{0}$ be an AFD factor of type $\mathrm{II}_{1}$ and $\beta$ a free action of the group $\widetilde{H}=H \times \mathrm{R}$ on $\mathcal{R}_{0}$. For each $x \in X$, consider the $\mu_{x}$-twisted crossed product

$$
\mathcal{R}_{\lambda, \mu}(x)=\mathcal{R}_{0} \rtimes_{\beta, \mu_{x}} L, \quad x \in X,
$$

where $\mu_{x}(m, n)=\tilde{\mu}(x ; m, n), m, n \in L$. Let $\{u(x ; m): m \in L\}, x \in X$, be the $\mu_{x}$-projective unitary representation of $L$ in $\mathcal{R}_{\lambda, \mu}(x)$ associated with the twisted crossed product. Then we have

$$
\mathcal{R}_{\lambda, \mu}(x)=\mathcal{R}_{0} \vee\{u(x ; m): m \in L\}^{\prime \prime}, \quad x \in X .
$$

For each $\tilde{g}=(y, \tilde{g}, x) \in \mathscr{G}$, set

$$
\begin{aligned}
\beta_{(y, \tilde{g}, x)}^{\lambda, \mu}(a) & =\beta_{\tilde{g}}(a) \quad \text { for } \quad a \in \mathcal{R}_{0} ; \\
\beta_{(y, \tilde{g}, x)}^{\lambda, \mu}(u(x ; m)) & =\tilde{\lambda}\left(y ; g m g^{-1} ; y, \tilde{g}, x\right) u\left(y ; g m g^{-1}\right), \quad m \in L .
\end{aligned}
$$

It is routine to check that $\beta^{\lambda, \mu}$ is indeed an action of the groupoid $\mathscr{G}$ on the Borel field $\left\{\mathcal{R}_{\lambda, \mu}(x): x \in X\right\}$ of factors of type $\mathrm{II}_{1}$ and the von Neumann algebra:

$$
\mathcal{M}_{0}(\lambda, \mu)=\int_{X}^{\oplus} \mathcal{R}_{\lambda, \mu}(x) \operatorname{dm}(x)
$$

accommodates the required action $\beta^{\lambda, \mu}$ of $\widetilde{H}$.

If $H$ is amenable, then $L$ is also amenable, which makes each $\mathcal{R}_{\lambda, \mu}(x)$ approximately finite dimensional, and therefore $\mathcal{M}_{0}$ is AFD.

THEOREM 2.3. Let $\{\mathcal{C}, \mathrm{R}, \theta\}$ be an ergodic flow and $\alpha$ an action of a countable discrete group $H$ on the flow, i.e., $\alpha$ is a homomorphism of $H$ into the 
group $\operatorname{Aut}_{\theta}(\mathcal{C})$ of automorphisms of $\mathcal{C}$ commuting with the flow $\theta$. Let $L$ be a normal subgroup of $H$ contained in the kernel $\operatorname{Ker}(\alpha)$ of $\alpha$, i.e., L does not act on $\mathcal{C}$ at all. Consider the product group $\widetilde{H}=H \times \mathrm{R}$ whose joint action on $\mathcal{C}$ will be denoted by $\alpha$. Then we have a functorial model construction:

$$
(\lambda, \mu) \in \mathrm{Z}_{\alpha}(\tilde{H}, L, \mathcal{U}(\mathcal{C})) \mapsto\left\{\widetilde{\mathcal{M}}(\lambda, \mu), \tilde{\alpha}^{\lambda, \mu}\right\}
$$

such that

i) The restriction of the covariant system $\left\{\tilde{\mathcal{M}}(\lambda, \mu), \widetilde{H}, \tilde{\alpha}^{\lambda, \mu}\right\}$ to the center is conjugate to $\{\mathcal{C}, \widetilde{H}, \alpha\}$;

ii) The von Neumann algebra $\widetilde{\mathcal{M}}(\lambda, \mu)$ is of type $\mathrm{II}_{\infty}$ and admits a faithful semi finite normal trace $\tau$ which is transformed in the following fashion:

$$
\tau \circ \alpha_{g, s}=e^{-s} \tau, \quad(g, s) \in \tilde{H}
$$

iii) $L=\left(\tilde{\alpha}^{\lambda, \mu}\right)^{-1}(\operatorname{Int}(\tilde{\mathcal{M}}(\lambda, \mu)))$ and $\tilde{\mathcal{M}}(\lambda, \mu)$ admits a map $u^{\lambda, \mu}: m \in$ $L \mapsto u^{\lambda, \mu}(m) \in U(\widetilde{\mathcal{M}})$ such that

$$
\begin{aligned}
u^{\lambda, \mu}(m) u^{\lambda, \mu}(n) & =\mu(m, n) u^{\lambda, \mu}(m n), \quad m, n \in L ; \\
\tilde{\alpha}_{g, s}^{\lambda, \mu}\left(u^{\lambda, \mu}(m)\right) & =\lambda\left(g m g^{-1} ; g, s\right) u^{\lambda, \mu}\left(g m g^{-1}\right), \quad(g, s) \in \widetilde{H} ;
\end{aligned}
$$

iv) To each $f \in \underset{\sim}{\operatorname{Map}}(L, \mathcal{U}(\mathcal{C}))$, there corresponds an isomorphism $\sigma_{f}$ : $\widetilde{\mathcal{M}}(\lambda, \mu) \mapsto \widetilde{\mathcal{M}}\left(\lambda \partial_{1} f, \mu \partial_{2} f\right)$ such that

$$
\begin{aligned}
& \sigma_{f}\left(u^{\lambda, \mu}(m)\right)=f(m) u^{\lambda, \mu}(m), \quad m \in L ; \\
& \sigma_{f} \circ \tilde{\alpha}_{g, s}^{\lambda, \mu} \circ \sigma_{f}^{-1}=\tilde{\alpha}_{g, s}^{\lambda \partial_{1} f, \mu \partial_{2} f}, \quad(g, s) \in \widetilde{H} ; \\
& \sigma_{f_{1}} \circ \sigma_{f_{2}}=\sigma_{f_{1} f_{2}}, \quad f_{1}, f_{2} \in \operatorname{Map}(L, \mathcal{C}(\mathcal{C})) ; \\
& \tau \circ \sigma_{f}=\tau, \quad f \in \operatorname{Map}(L, U(\mathcal{C})) .
\end{aligned}
$$

Consequently, the restriction $\alpha^{\lambda, \mu}$ of $\tilde{\alpha}^{\lambda, \mu}$ to the fixed point subalgebra:

$$
\mathcal{M}(\lambda, \mu)=\tilde{\mathcal{M}}(\lambda, \mu)^{\theta}
$$

is an action of the group $H$ on a factor of type III whose flow of weights is precisely $\{\mathcal{C}, \mathrm{R}, \theta\}$ such that its modular characteristic invariant is given by:

$$
\chi_{\mathrm{m}}\left(\alpha^{\lambda, \mu}\right)=[\lambda, \mu] \in \Lambda_{\alpha}(\tilde{H}, L, U(\mathcal{C})) .
$$

If the group $H$ is amenable, then the factor $\mathcal{M}$ of the model $\left\{\mathcal{M}(\lambda, \mu), H, \alpha^{\lambda, \mu}\right\}$ is approximately finite dimensional.

Proof. We continue the discussion in the proof of Lemma 2.2. Let $\mathcal{R}_{0,1}$ be an AFD factor of type $\mathrm{II}_{\infty}$ equipped with a one parameter automorphism 
group $\left\{\theta_{s}: s \in \mathrm{R}\right\}$ scaling trace, i.e., $\tau \circ \theta_{s}=e^{-s} \tau, s \in \mathrm{R}$, with $\tau$ a faithful semi-finite normal trace on $\mathcal{R}_{0,1}$. We set

$$
\begin{gathered}
\tilde{\mathcal{M}}(\lambda, \mu)=\mathcal{M}_{0}(\lambda, \mu) \bar{\otimes} \mathcal{R}_{0,1} \quad \text { and } \quad \tilde{\mathcal{M}}(\lambda, \mu ; x)=\mathcal{R}_{\lambda, \mu}(x) \bar{\otimes} \mathcal{R}_{0,1} ; \\
\widetilde{\mathcal{M}}(\lambda, \mu)=\int_{X}^{\oplus} \tilde{\mathcal{M}}(\lambda, \mu ; x) \operatorname{dm}(x) .
\end{gathered}
$$

Let $\delta_{\mathrm{m}}$ be the modulus of the quasi-invariant measure $\mathrm{m}$ on $X$ relative to the groupoid $\mathscr{G}$, i.e.,

$$
\delta_{\mathrm{m}}(y, \tilde{g}, x)=\frac{\mathrm{d}\left(\mathrm{m} \circ \tilde{g}^{-1}\right)}{\mathrm{dm}}(x), \quad(y, \tilde{g}, x) \in \mathscr{G},
$$

and set

$$
\rho_{\mathrm{m}}(\tilde{g})=s+\log \left(\delta_{\mathrm{m}}(\tilde{g})\right), \quad \tilde{g} \in \mathscr{G} .
$$

We then define an action $\left\{\alpha_{(y, \tilde{g}, x)}^{\lambda, \mu}\right\}$ of $\mathscr{G}$ on the field $\{\widetilde{\mathcal{M}}(\lambda, \mu ; x): x \in X\}$ in the following fashion: with $\tilde{g}=(y, \tilde{g}, x), \tilde{g}=(g, s) \in \widetilde{H}=H \times \mathrm{R}$, $\widetilde{\mathcal{M}}(\lambda, \mu ; x)=\mathcal{R}_{\lambda, \mu}(x) \bar{\otimes} \mathcal{R}_{0,1} \frac{\beta_{(y, \tilde{g}, x)}^{\lambda, \mu} \otimes \theta_{\rho_{\mathrm{m}}(y, \tilde{g}, x)}}{\alpha_{(y, \tilde{g}, x)}^{\lambda, \mu}} \widetilde{\mathcal{M}}(\lambda, \mu ; y)=\mathcal{R}_{\lambda, \mu}(y) \bar{\otimes} \mathcal{R}_{0,1}$. Then we have for each $a \in \tilde{\mathcal{M}}(\lambda, \mu)$ and $\tilde{g}=(g, s) \in \widetilde{H}$

$$
\begin{aligned}
\tau \circ \alpha_{\tilde{g}}^{\lambda, \mu}(a) & =\int_{X} \tau_{y}\left(\left(\alpha_{\tilde{g}}^{\lambda, \mu}(a)\right)(y)\right) \mathrm{dm}(y) \\
& =\int_{X} \tau_{y}\left(\alpha_{(y, \tilde{g}, x)}^{\lambda, \mu}(a(x))\right) \mathrm{dm}(y) \\
& =\int_{X} \frac{\mathrm{d} \tau_{y} \circ \alpha_{(y, \tilde{g}, x)}}{\mathrm{d} \tau_{x}} \tau_{x}(a(x)) \delta_{\mathrm{m}}(y, \tilde{g}, x) \mathrm{dm}(x) \\
& =\int_{X} e^{-\rho_{\mathrm{m}}(y, \tilde{g}, x)} \tau_{x}(a(x)) \delta_{\mathrm{m}}(y, \tilde{g}, x) \mathrm{dm}(x) \\
& =\int_{X} e^{-s-\log \left(\delta_{\mathrm{m}}(y, \tilde{g}, x)\right)} \tau_{x}(a(x)) \delta_{\mathrm{m}}(y, \tilde{g}, x) \mathrm{dm}(x) \\
& =\int_{X} e^{-s} \tau_{x}(a(x)) \mathrm{dm}(x)=e^{-s} \tau(a) .
\end{aligned}
$$

Therefore the trace transformation rule of the integrated action $\widetilde{\alpha}^{\lambda, \mu}$ of $\widetilde{H}$ on $\widetilde{\mathcal{M}}(\lambda, \mu)$ :

$$
\tau \circ \widetilde{\alpha}_{g, s}=e^{-s} \tau, \quad(g, s) \in \widetilde{H},
$$


follows. The rest of the assertions in the theorem follow easily now. This completes the proof.

Corollary 2.4. Suppose that $G$ is a countable discrete group with a preassigned normal subgroup $N$ and that $\{\mathcal{C}, \mathbf{R}, \theta\}$ is an ergodic flow. Let $\alpha$ be an action of $G$ on the flow $\{\mathcal{C}, \mathrm{R}, \theta\}$ such that $N \subset \operatorname{Ker}(\alpha)$, i.e., a homomorphism of $G$ into the group $\operatorname{Aut}_{\theta}(\mathcal{C})$ of automorphisms commuting with $\theta$ which maps $N$ to the identity. So the action $\alpha$ factors through the quotient group $Q=G / N$. Fix a cross-section $\mathfrak{g}: Q \mapsto G$ of the quotient map $\pi: g \in G \mapsto g N \in Q$. Then for any modular obstruction cocycle $[c, v] \in \mathrm{H}_{\alpha, \mathscr{\xi}}^{\text {out }}(\widetilde{G}, N, \mathcal{U}(\mathcal{C}))$, there exists an outer action $\alpha$ of $G$ on an infinite factor $\mathcal{M}$ with $N=\alpha^{-1}\left(\mathrm{Cnt}_{\mathrm{r}}(\mathcal{M})\right)$ such that the associated modular obstruction $\mathrm{Ob}_{\mathrm{m}}(\alpha)$ is precisely the cohomology class $\mathrm{Ob}_{\mathrm{m}}(\alpha)=[\xi, \nu] \in \mathrm{H}_{\alpha, \mathfrak{\xi}}^{\text {out }}(\widetilde{G}, N, \mathcal{U}(\mathcal{C}))$.

If $G$ is amenable, then the construction of $\{\mathcal{M}, G, \alpha\}$ yields that $\mathcal{M}$ is approximately finite dimensional.

Proof. Denote the unitary group $U(\mathcal{C})$ by $A$ for short and $\widetilde{H}=H \times \mathrm{R}$. Let $[c]=\partial([\xi, \zeta]) \in \mathrm{H}_{\widetilde{G}}^{3}(G, \mathrm{~T})$ be the image of the cohomology class $[\xi, \nu]$ under the map $\partial: \mathrm{H}_{\alpha, \breve{\xi}}^{\text {out }}(\widetilde{G}, N, A) \mapsto \mathrm{H}^{3}(G, \mathrm{~T})$ of [17, Lemma 2.11]. Consider the resolution system:

$$
1 \longrightarrow M \longrightarrow H(c) \stackrel{\pi_{G}}{\longrightarrow} G \longrightarrow 1
$$

of the cocycle $c \in \mathrm{Z}^{3}(G, \mathrm{~T})$. As $\pi_{G}^{*}([c])=1$ in $\mathrm{H}^{3}(H, \mathrm{~T})$, we have $\operatorname{Inf}([\xi, v])$ $=\pi_{G}^{*} \circ \partial([\xi, v])=1 \in \mathrm{H}^{3}(H, \mathrm{~T})$ in the modified HJR-exact sequence, so that we can find $\chi=[\lambda, \mu] \in \Lambda_{\alpha}(\widetilde{H}, L, A)$ such that $[\xi, \nu]=\delta(\chi)$ by [17, Theorem 2.7]. Now Theorem 2.3 yields the existence of a covariant system $\left\{\mathcal{M}, H, \alpha^{\lambda, \mu}\right\}$ such that $L=\left(\alpha^{\lambda, \mu}\right)^{-1}\left(\operatorname{Cnt}_{\mathrm{r}}(\mathcal{M})\right)$ and $\chi(\alpha)=\chi$. With $\Im_{H}$ a cross-section of $\pi_{G}$, we set

$$
\alpha_{g}=\alpha_{\mathfrak{S}_{H}(g)}^{\lambda, \mu} \quad \text { on } \quad \mathcal{M}, g \in G,
$$

to obtain the outer action $\alpha$ of $G$ on $\mathcal{M}$ such that $\operatorname{Ob}_{\mathrm{m}}(\alpha)=[\xi, \nu]$. This completes the proof.

\section{Reduction of Invariants for the Case of Type $\mathrm{III}_{\lambda}, 0<\lambda<1$}

First, fixing $0<\lambda<1$, we set

$$
T=-2 \pi /(\log \lambda)>0 \quad \text { and } \quad T^{\prime}=-\log \lambda>0 .
$$

Let $\mathcal{C}=L^{\infty}\left(\mathrm{R} / T^{\prime} \mathrm{Z}\right)$ and $A=U(\mathcal{C})$. The action $\theta$ of the real line $\mathrm{R}$ on $\mathrm{T}=\mathrm{R} / T^{\prime} \mathrm{Z}$ is by translation. 
LEMMA 3.1. In the above context, the first cohomology group $\mathrm{H}_{\theta}^{1}(\mathrm{R}, A)$ has the following structure:

i) $\mathrm{H}_{\theta}^{1}(\mathrm{R}, A) \cong \mathrm{R} / T \mathrm{Z}$;

ii) The following exact sequence splits:

$$
1 \longrightarrow \mathrm{B}_{\theta}^{1}(\mathrm{R}, A) \longrightarrow \mathrm{Z}_{\theta}^{1}(\mathrm{R}, A) \underset{\mathfrak{g}_{\mathrm{Z}}}{\rightleftarrows} \mathrm{R} / T \mathrm{Z} \longrightarrow 0
$$

Proof. i) This follows from Proposition 1.8 by setting $Y=\{0\} \in X=$ $\mathrm{R} / T^{\prime} \mathrm{Z}$. Because

$$
\mathscr{G}_{Y}=\{0\} \times T^{\prime} \mathrm{Z}, A(0)=\mathrm{T} \quad \text { and } \quad \mathrm{H}_{\theta}^{1}\left(T^{\prime} \mathrm{Z}, \mathrm{T}\right)=\widehat{T^{\prime} \mathrm{Z}} \cong \mathrm{R} / T \mathrm{Z} .
$$

ii) The notation $[x], x \in \mathrm{R}$, stands for the Gauss symbol, i.e., the unique integer $n$ such that $n \leq x<n+1$. Set

$$
c(s, t, x)=\exp \left(\mathrm{i} T^{\prime} s\left(\left[\frac{x}{T^{\prime}}\right]-\left[\frac{x+t}{T^{\prime}}\right]\right)\right), \quad s, t, x \in \mathrm{R},
$$

and observe that the function $c(s, t, \cdot)$ is periodic in the last variable $x$ with period $T^{\prime}$, so that it can be viewed as a function over $X=\mathrm{R} / T^{\prime} Z$. Furthermore, we have

$$
\begin{aligned}
c(r+s, t, x) & =c(r, t, x) c(s, t, x) \\
c(r, s+t, x) & =c(r, s, x) c(r, t, x+s) \\
c(T, t, x) & =1 .
\end{aligned}
$$

Thus $\{c(s, \cdot, \cdot)\}$ is a one parameter subgroup of $\mathrm{Z}_{\theta}^{1}(\mathrm{R}, A)$ with period $T$. Hence the map: $\dot{s}_{T} \in \mathrm{R} / T \mathrm{Z} \mapsto c\left(\dot{s}_{T}, \cdot, \cdot\right) \in \mathrm{Z}_{\theta}^{1}(\mathrm{R}, A)$ is a cross-section $\mathfrak{s}_{\mathrm{Z}}$ of the $\operatorname{map} \pi_{\mathrm{Z}}: \mathrm{Z}_{\theta}^{1}(\mathrm{R}, A) \mapsto \mathrm{H}_{\theta}^{1}(\mathrm{R}, A)=\mathrm{R} / T \mathrm{Z}$.

Corollary 3.2. If $\mathcal{M}$ is a separable factor of type $\mathrm{III}_{\lambda}, 0<\lambda<1$, then the association of extended modular automorphism to each $\dot{s} \in \mathrm{R} / T \mathrm{Z}$ with $T=-2 \pi /(\log \lambda)$ and $\varphi \in \mathfrak{W}_{0}(\mathcal{M})$ :

$$
(\dot{s}, \varphi) \in \mathrm{R} / T \mathrm{Z} \times \mathfrak{W}_{0}(\mathcal{M}) \mapsto \sigma_{\mathfrak{s}_{\mathrm{Z}}\left(\dot{s}_{T}\right)}^{\varphi} \in \mathrm{Cnt}_{\mathrm{r}}(\mathcal{M})
$$

is equivariant in the sense that if $\alpha$ is an isomorphism of $\mathcal{M}$ onto another factor $\mathcal{N}$, then

$$
\alpha \circ \sigma_{\mathfrak{g}_{\mathrm{Z}}\left(\dot{s}_{T}\right)}^{\varphi} \circ \alpha^{-1}=\sigma_{\alpha\left(\mathfrak{\Xi}_{\mathrm{Z}}\left(\dot{s}_{T}\right)\right)}^{\alpha(\varphi)}, \quad \dot{s}_{T} \in \mathrm{R} / T \mathrm{Z} .
$$

In fact, for each $\varphi \in \mathfrak{W}_{0}(\mathcal{M})$, there exists a non-singular positive $H \in \mathcal{C}_{\varphi}$ such that with $\rho=\varphi(H \cdot)$,

$$
\sigma_{s}^{\rho}=\sigma_{\mathfrak{g}_{\mathrm{Z}}\left(\dot{s}_{T}\right)}^{\varphi}, \quad s \in \mathbf{R},
$$


where $\dot{s}_{T}=s+T \mathrm{Z} \in \mathrm{R} / T \mathrm{Z}$.

Proof. The last lemma shows that $\left\{\mathfrak{s}_{Z}\left(\dot{s}_{T}\right)\right\}$ is a one parameter subgroup of $\mathrm{Z}_{\theta}^{1}(\mathrm{R}, A)$ with period $T$. Hence for each $\varphi \in \mathfrak{S}_{0}(\mathcal{M}),\left\{u_{\varphi}\left(\dot{s}_{T}\right): s \in \mathbf{R}\right\}=$ $\left\{b_{\varphi}\left(\mathfrak{s}_{Z}\left(\dot{s}_{T}\right)\right): s \in \mathrm{R}\right\}$ is a one parameter subgroup of $\tilde{U}(\mathcal{M}) \cap D^{\varphi}$ with period $T$ (for notations, see [28, p. 458]) and $\left\{\sigma_{\tilde{\xi}_{Z}\left(\dot{s}_{T}\right)}^{\varphi}: s \in \mathrm{R}\right\}$ is a one parameter automorphism group of $\mathcal{M}$ with period $T$ which leaves $\mathcal{M}_{\varphi}$ pointwise invariant.

Fix $\varphi \in \mathfrak{W}_{0}(\mathcal{M})$ and take a non-singular positive $k \in \mathcal{C}_{\varphi}$ such that $\operatorname{Ad}\left(k^{-i T}\right)$ $=\sigma_{T}^{\varphi}$, so that $\psi=\varphi(k \cdot)$ is a faithful semi-finite normal weight with $\sigma_{T}^{\psi}=$ id. Then it follows that $\psi^{\mathrm{i} T}$ belongs to the center $\mathcal{C}$, (in fact it generates $\mathcal{C}$ ). With $h \in \mathcal{C}$ such that $h^{\mathrm{i} T}=\psi^{\mathrm{i} T}$, we have

$$
\partial_{\theta}\left(h^{-i s} k^{i s} \varphi^{i s}\right)=\partial_{\theta}\left(h^{-i s} \psi^{i s}\right)=\mathfrak{s}_{\mathrm{Z}}\left(\dot{s}_{T}\right), \quad s \in \mathbf{R} .
$$

Hence we get

$$
\partial_{\theta}\left(u_{\varphi}\left(\dot{s}_{T}\right)\right)=\mathfrak{s}_{\mathrm{Z}}\left(\dot{s}_{T}\right)=\partial_{\theta}\left(h^{-i s} k^{i s} \varphi^{i s}\right), \quad s \in \mathbf{R} .
$$

This means that $v(s)=u_{\varphi}\left(\dot{s}_{T}\right) h^{i s} k^{-i s} \varphi^{-i s} \in \mathcal{U}(\mathcal{M}), s \in \mathrm{R}$. Now as $k \in \mathcal{C}_{\varphi}$, $v(s)$ and $\mathscr{D}^{\varphi}$ commute, consequently so do $\varphi$ and $v(s)$. Hence $\{v(s)\}$ is a periodic one parameter unitary group in the centralizer $\mathcal{M}_{\varphi}$ with period $T$ such that

$$
\sigma_{\tilde{\mathcal{F}}_{\mathrm{Z}}\left(\dot{s}_{T}\right)}^{\varphi}=\operatorname{Ad}(v(s)) \circ \sigma_{s}^{\psi}, \quad s \in \mathrm{R} .
$$

Since both $\sigma_{\mathfrak{S}_{\mathrm{Z}}\left(\dot{s}_{T}\right)}^{\varphi}$ and $\sigma_{s}^{\psi}$ leave $\mathcal{M}_{\varphi}$ pointwise invariant, $\{v(s)\}$ is contained in the center $\mathcal{C}_{\varphi}$. Thus there exists a non-singular $\ell \in \mathcal{C}_{\varphi}$ such that $v(s)=\ell^{i s}$. Therefore the operator $H=k \ell \in \mathcal{C}_{\varphi}$ gives a faithful semi-finite normal weight $\rho$ such that $\sigma_{s}^{\rho}=\sigma_{\mathfrak{g}_{\mathrm{Z}}\left(\dot{s}_{T}\right)}^{\varphi}, s \in \mathrm{R}$, as required.

The equivariance of $\sigma_{\mathfrak{s}_{\mathrm{Z}}\left(\dot{s}_{T}\right)}^{\varphi}$ follows from that of the Falcone-Takesaki crosssection $b_{\varphi}$ :

$$
\begin{aligned}
\sigma_{\mathfrak{g}_{\mathrm{Z}}(\dot{s})}^{\varphi} & =\widetilde{\operatorname{Ad}}\left(b_{\varphi}\left(\mathfrak{s}_{\mathrm{Z}}(\dot{s})\right)\right), \quad \dot{s} \in \mathrm{R} / T \mathrm{Z} ; \\
\alpha\left(b_{\varphi}\left(\mathfrak{g}_{\mathrm{Z}}(\dot{s})\right)\right. & =b_{\varphi^{\circ} \alpha^{-1}}\left(\alpha\left(\mathfrak{s}_{\mathrm{Z}}(\dot{s})\right)\right) .
\end{aligned}
$$

This completes the proof.

THEOREM 3.3. If $\mathcal{M}$ is a separable factor of type $\mathrm{III}_{\lambda}$, then the intrinsic invariant $\Theta(\mathcal{M})$ lives in the group:

$$
\Lambda\left(\operatorname{Aut}(\mathcal{M})_{\mathrm{m}}, \operatorname{Cnt}(\mathcal{M}), \mathrm{T}\right) \cong \Lambda_{\bmod \times \theta}\left(\operatorname{Aut}(\mathcal{M}) \times \mathrm{R}, \mathrm{Cnt}_{\mathrm{r}}(\mathcal{M}), \mathcal{U}(\mathcal{C})\right),
$$

where

$$
\operatorname{Aut}(\mathcal{M})_{\mathrm{m}}=\left\{(\alpha, s) \in \operatorname{Aut}(\mathcal{M}) \times \mathrm{R}: \bmod (\alpha)=\dot{s}_{T^{\prime}} \in \mathrm{R} / T^{\prime} \mathrm{Z}\right\},
$$


and $\{\mathcal{C}, \mathrm{R}, \theta\}$ is the flow of weights on $\mathcal{M}$, i.e., $\mathcal{C}=L^{\infty}\left(\mathrm{R} / T^{\prime} \mathrm{Z}\right)$ and $\theta$ is the translation with $T^{\prime}=-\log \lambda$.

Proof. This is simply restating Proposition 1.11 with $Y=\{0\} \in X=$ $\mathrm{R} / T^{\prime} \mathrm{Z}$. We leave the detail to the reader.

Before going any further, we need to discuss the structure of the third cohomology group $\mathrm{H}^{3}\left(Q_{\mathrm{m}}, \mathrm{T}\right)$. So let $Q_{\mathrm{m}}$ be a discrete group equipped with a distinguished torsion free central element $z_{0}$. We denote by $Z$ the cyclic subgroup generated by $z_{0}$, which is isomorphic to the integer group $\mathbf{Z}$ under the map: $n \in \mathbf{Z} \mapsto z_{0}^{n} \in Z$. We denote the quotient group $Q_{\mathrm{m}} / Z$ by $Q$. The elements of $Q_{\mathrm{m}}$ are denoted by $\tilde{p}, \tilde{q}, \tilde{r}$ and so on and their quotient images will be denoted by plain $p, q, r$ and so on. The quotient map: $Q_{\mathrm{m}} \mapsto Q$ will be denoted by $\pi_{\mathrm{m}}$, so $p=\pi_{\mathrm{m}}(\tilde{p}), q=\pi_{\mathrm{m}}(\tilde{q}), r=\pi_{\mathrm{m}}(\tilde{r})$. A cross-section $\mathfrak{S}_{\mathrm{m}}$ of the exact sequence:

$$
1 \longrightarrow Z \longrightarrow Q_{\mathrm{m}} \underset{\mathfrak{s}_{\mathrm{m}}}{\stackrel{\pi_{\mathrm{m}}}{\rightleftarrows}} Q \longrightarrow 1
$$

gives rise to an element $n \in \mathrm{Z}^{2}(Q, \mathrm{Z})$ such that

$$
\mathfrak{F}_{\mathrm{m}}(p) \mathfrak{F}_{\mathrm{m}}(q)=z_{0}^{n(p, q)} \mathfrak{F}_{\mathrm{m}}(p q), \quad p, q \in Q .
$$

Definition 3.4. A cocycle $c \in \mathrm{Z}^{3}\left(Q_{\mathrm{m}}, \mathrm{T}\right)$ is said to be standard if there exists a $d_{c} \in \mathrm{C}^{2}(Q, \mathrm{~T})$ such that

$$
c\left(\tilde{p} z_{0}^{m}, \tilde{q} z_{0}^{n}, \tilde{r} z_{0}^{\ell}\right)=d_{c}(q, r)^{m} c(\tilde{p}, \tilde{q}, \tilde{r})
$$

for each $m, n, \ell \in \mathrm{Z}$ and $\tilde{p}, \tilde{q}, \tilde{r} \in Q_{\mathrm{m}}$. We denote the group of all standard 3-cocycles by $\mathrm{Z}_{\mathrm{s}}^{3}\left(Q_{\mathrm{m}}, \mathrm{T}\right)$. The cochain $d_{c}$ is called the $d$-part of the standard cocycle $c \in \mathrm{Z}_{\mathrm{s}}^{3}\left(Q_{\mathrm{m}}, \mathrm{T}\right)$.

Needless to say, the above definition makes sense only when we fix the distinguished element $z_{0}$ in the center of $Q_{\mathrm{m}}$. This element will stand for the automorphism $\theta_{T^{\prime}}$ on the discrete core $\widetilde{\mathcal{M}}_{d}$ of a factor $\mathcal{M}$ of type III $_{\lambda}$ which scales the trace by $\lambda$.

THEOREM 3.5. i) Each $c \in \mathrm{Z}^{3}\left(Q_{\mathrm{m}}, \mathrm{T}\right)$ is cohomologous to a standard cocycle $c_{\mathrm{s}} \in \mathrm{Z}_{\mathrm{s}}^{3}\left(Q_{\mathrm{m}}, \mathrm{T}\right)$.

ii) The $d$-part $d_{c}$ of a standard cocycle $c \in Z_{\mathrm{s}}^{3}\left(Q_{\mathrm{m}}, \mathrm{T}\right)$ is necessarily a cocycle, i.e., $d_{c} \in \mathrm{Z}^{2}(Q, \mathrm{~T})$ and c-part $c(\tilde{p}, \tilde{q}, \tilde{r})$ satisfies;

$$
c\left(z_{0}, \tilde{q}, \tilde{r}\right)=d_{c}(q, r) .
$$


iii) A standard cocycle $c \in \mathrm{Z}^{3}\left(Q_{\mathrm{m}}, \mathrm{T}\right)$ is a coboundary if and only if there is a function $f \in \mathrm{C}^{2}\left(Q_{\mathrm{m}}, \mathrm{T}\right)$ with $c=\partial_{Q_{\mathrm{m}}} f$ satisfying

$$
\left\{\begin{array}{l}
f\left(\tilde{p} z_{0}^{m}, \tilde{q} z_{0}^{n}\right)=f\left(z_{0}, q\right)^{m} f(\tilde{p}, \tilde{q}) \\
f\left(z_{0}^{n}, q\right) f\left(z_{0}^{m}, q\right)=f\left(z_{0}^{m+n}, q\right) \\
f(\tilde{p}, 1)=f(1, \tilde{q})=1
\end{array}\right.
$$

for all $\tilde{p}, \tilde{q} \in Q_{\mathrm{m}}$.

More precisely, there exists an element $f \in \mathrm{C}^{2}\left(Q_{\mathrm{m}}, \mathrm{T}\right)$ such that the function $f(\tilde{p}, \cdot)$ of the second variable factors through the quotient map $\pi_{\mathrm{m}}$ and

$$
\begin{aligned}
& c(\tilde{p}, \tilde{q}, \tilde{r})=\left(\partial_{Q_{\mathrm{m}}} f\right)(\tilde{p}, \tilde{q}, \tilde{r}) ; \\
& d_{c}(q, r)=f\left(z_{0}, r\right) f\left(z_{0}, q\right) f\left(z_{0}, q r\right)^{-1} ; \\
& f\left(\tilde{p} z_{0}^{m}, \tilde{q} z_{0}^{n}\right)=f\left(z_{0}, q\right)^{m} f(\tilde{p}, \tilde{q}) ; \\
& f\left(z_{0}^{n}, q\right) f\left(z_{0}^{m}, q\right)=f\left(z_{0}^{m+n}, q\right)
\end{aligned}
$$

for any pair $q, r \in Q$ and $\tilde{q}, \tilde{r} \in Q_{\mathrm{m}}$ with $q=\pi_{\mathrm{m}}(\tilde{q}), r=\pi_{\mathrm{m}}(\tilde{r})$.

Choose and fix a cocycle $c \in \mathrm{Z}^{3}\left(Q_{\mathrm{m}}, \mathrm{T}\right)$. Consider the von Neumann algebra $\mathcal{A}=\ell^{\infty}\left(Q_{\mathrm{m}}\right)$ of bounded functions over $Q_{\mathrm{m}}$ and let $B$ be the unitary group $B=U(\mathcal{A})=\mathrm{T}_{\mathrm{m}}^{Q}$, which generates an exact sequence of compact abelian groups:

$$
1 \longrightarrow \mathrm{T} \stackrel{i}{\longrightarrow} B \underset{\mathfrak{g}_{j}}{\stackrel{j}{\rightleftarrows}} C \longrightarrow 1
$$

The exact sequence splits so that the group $C$ is identified with the subgroup of $B$ consisting of all T-valued functions on $Q_{\mathrm{m}}$ whose value at the identity $1 \in Q_{\mathrm{m}}$ is 1 . The map $j$ is given by:

$$
(j f)(\tilde{p})=\frac{f(\tilde{p})}{f(1)}, \quad f \in B .
$$

The translation action of $Q_{\mathrm{m}}$ does not leave the subgroup $C$ invariant, but the little twisted action given by

$$
\left(\alpha_{\tilde{p}} f\right)(\tilde{q})=\frac{f(\tilde{q} \tilde{p})}{f(\tilde{p})}, \quad f \in C,
$$

is consistent with the right translation action $\alpha$ of $Q_{\mathrm{m}}$ on $B$ as seen below:

$$
\left(j \circ \alpha_{\tilde{p}}(f)\right)(\tilde{q})=\frac{\left(\alpha_{\tilde{p}} f\right)(\tilde{q})}{\left(\alpha_{\tilde{p}} f\right)(1)}=\frac{f(\tilde{q} \tilde{p})}{f(\tilde{p})}=\left(\alpha_{\tilde{p}} \circ j(f)(\tilde{q}), \quad f \in C .\right.
$$


The action $\alpha_{z_{0}}$ will be denoted by $\theta$. The fixed point subgroup $C^{\theta}$ will be denoted by $L$, which is the subgroup consisting of those functions $f \in C$ such that

$$
\partial_{\theta} f=(\theta f) f^{-1}=\text { Constant } \in \mathrm{T},
$$

equivalently

$$
f\left(\tilde{p} z_{0}\right)=f(\tilde{p}) f\left(z_{0}\right), \quad \tilde{p} \in Q_{\mathrm{m}} .
$$

Therefore, on $E=j^{-1}(L)$, the coboundary operator $\partial_{\theta}$ becomes the evaluation of the value of a function $f \in E$ at $z_{0}$, i.e.,

$$
\partial_{\theta} f=f\left(z_{0}\right), \quad f \in E .
$$

The cocycle $i_{*} c \in \mathrm{Z}_{\alpha}^{3}\left(Q_{\mathrm{m}}, B\right)$ is cobounded by the element $u=u_{c} \in$ $\mathrm{C}_{\alpha}^{2}\left(Q_{\mathrm{m}}, B\right)$ defined by:

$$
u(x ; \tilde{p}, \tilde{q})=c(x, \tilde{p}, \tilde{q}), \quad x, \tilde{p}, \tilde{q} \in Q_{\mathrm{m}},
$$

as seen below:

$$
\begin{aligned}
1 & =c(\tilde{p}, \tilde{q}, \tilde{r}) \overline{c(x \tilde{p}, \tilde{q}, \tilde{r})} c(x, \tilde{p} \tilde{q}, \tilde{r}) \overline{c(x, \tilde{p}, \tilde{q} \tilde{r})} c(x, \tilde{p}, \tilde{q}) \\
c(\tilde{p}, \tilde{q}, \tilde{r}) & =c(x \tilde{p}, \tilde{q}, \tilde{r}) \overline{c(x, \tilde{p} \tilde{q}, \tilde{r})} c(x, \tilde{p}, \tilde{q} \tilde{r}) \overline{c(x, \tilde{p}, \tilde{q})} \\
& =\left(\alpha_{\tilde{p}} u\right)(x ; \tilde{q}, \tilde{r}) u(x ; \tilde{p} \tilde{q}, \tilde{r})^{-1} u(x ; \tilde{p}, \tilde{q} \tilde{r}) u(x ; \tilde{p}, \tilde{q})^{-1} \\
& =\left(\alpha_{\tilde{p}}(u(\tilde{q}, \tilde{r})) u(\tilde{p} \tilde{q}, \tilde{r})^{-1} u(\tilde{p}, \tilde{q} \tilde{r}) u(\tilde{p}, \tilde{q})^{-1}\right)(x) \\
& =\left(\partial_{Q_{\mathrm{m}}} u\right)(x ; \tilde{p}, \tilde{q}, \tilde{r}) .
\end{aligned}
$$

In fact, we have

$$
\mathrm{H}_{\alpha}^{n}\left(Q_{\mathrm{m}}, B\right)=\{1\}, \quad n=1,2, \ldots
$$

As $c(1, \tilde{q}, \tilde{r})=1$, the element $u(\tilde{q}, \tilde{r}) \in B, \tilde{q}, \tilde{r} \in Q_{\mathrm{m}}$, belongs to $C$. But to distinguish $u(\tilde{q}, \tilde{r}) \in C$ from $u(\tilde{q}, \tilde{r}) \in B$, we will denote $u(\tilde{q}, \tilde{r}) \in C$ by $j_{*} u(\tilde{q}, \tilde{r})$. Since $\left.\left(\partial_{Q_{\mathrm{m}}} j_{*} u(\tilde{p}, \tilde{q}, \tilde{r})\right)\right)=j(c(\tilde{p}, \tilde{q}, \tilde{r}))=1, j_{*}(u)$ is an element of $\mathrm{Z}_{\alpha}^{2}\left(Q_{\mathrm{m}}, C\right)$, which gives rise to an exact sequence:

$$
\begin{gathered}
1 \longrightarrow C \longrightarrow \rtimes_{\alpha, j_{*} u} Q_{\mathrm{m}} \stackrel{\dot{\pi}}{\rightleftarrows} Q_{\mathrm{m}} \longrightarrow 1 \\
(a, \tilde{p})(b, \tilde{q})=\left(a \alpha_{\tilde{p}}(b)\left(j_{*} u\right)(\tilde{p}, \tilde{q}), \tilde{p} \tilde{q}\right), \quad a, b \in C, \tilde{p}, \tilde{q} \in Q_{\mathrm{m}} \\
\dot{\pi}(a, \tilde{p})=\tilde{p}, \quad \mathfrak{s}(\tilde{p})=(1, \tilde{p}),
\end{gathered}
$$

so that

$$
j_{*}(u(\tilde{p}, \tilde{q}))=\mathfrak{n}_{C}(\tilde{p}, \tilde{q})=\mathfrak{s}(\tilde{p}) \mathfrak{g}(\tilde{q}) \mathfrak{s}(\tilde{p} \tilde{q})^{-1}, \quad \tilde{p}, \tilde{q} \in Q_{\mathrm{m}} .
$$


The action $\alpha_{\tilde{p}} \in \operatorname{Aut}(C)$ of $\tilde{p}$ on $C$ is given as the restriction of the inner automorphism $\operatorname{Ad}(\mathfrak{s}(\tilde{p})) \in \operatorname{Int}\left(C \rtimes_{\alpha, j_{*} u} Q_{\mathrm{m}}\right)$. The coherence of the action $\alpha_{\tilde{p}}$ on $B$ and $\operatorname{Ad}(\mathfrak{s}(\tilde{p}))$ on $C$ intertwined by $\dot{\pi}$ gives rise to an element $(\lambda, \mu) \in$ $\mathrm{Z}_{\alpha}\left(C \rtimes_{\alpha, j_{*} u} Q_{\mathrm{m}}, C, \mathrm{~T}\right)$ such that

$$
\begin{aligned}
\alpha_{\tilde{p}}\left(\mathfrak{S}_{j}\left(\mathfrak{s}(\tilde{p})^{-1} a \mathfrak{S}(\tilde{p})\right)\right) & =\lambda(a, \tilde{p}) \mathfrak{S}_{j}(a), \quad(a, \tilde{p}) \in C \rtimes_{\alpha, j_{*} u} Q_{\mathrm{m}} ; \\
\mu(a, b) & =1, \quad a, b \in C .
\end{aligned}
$$

The fixed point subalgebra $\mathscr{A}^{\theta}$ is identified with $\mathscr{B}=\ell^{\infty}(Q)$ and therefore the fixed point subgroup $B^{\theta}$ is identified with $U(\mathscr{B})=\mathrm{T}^{Q}$, the compact abelian group of all T-valued functions over $Q$. Set $K=B^{\theta} / i(\mathrm{~T}) \subset L=C^{\theta}$. We then have the following:

Lemma 3.6. There exists a cross-section $\dot{\dot{s}}: Q_{\mathrm{m}} \mapsto C \rtimes_{\alpha, j_{*}(u)} Q_{\mathrm{m}}$ such that the element $z=\mathfrak{s}\left(z_{0}\right)$ commutes with $\dot{\mathfrak{s}}\left(Q_{\mathrm{m}}\right)$. Hence the associated cocycle $\dot{\mathfrak{n}}_{L}=\partial_{Q_{\mathrm{m}}} \dot{\mathfrak{s}}$ takes values in $L$ and therefore $\dot{\mathfrak{n}}_{L} \in \mathrm{Z}_{\alpha}^{2}\left(Q_{\mathrm{m}}, L\right)$.

Proof. Since $H^{3}(Z, T)=\{1\}$, we may assume that $\left.c\right|_{Z^{3}}=1$. Hence the restriction $\left.u\right|_{\mathrm{Z}^{2}}$ is a cocycle. Hence there exists $v \in \mathrm{C}^{1}(\mathrm{Z}, B)$ such that $\left.u\right|_{\mathrm{Z}^{2}}=$ $\partial_{\mathrm{Z}} v$. Extending $v$ to $Q_{\mathrm{m}}$ and replacing $u$ by $\left(\partial_{Q_{\mathrm{m}}} v\right)^{-1} u$, we may and do assume that the restriction $\left.u\right|_{Z^{2}}$ of $u$ to $Z^{2}$ is trivial, i.e., $u\left(z_{0}^{m}, z_{0}^{n}\right)=1$. Hence we have $\mathfrak{s}\left(z_{0}^{m}\right)=\mathfrak{s}\left(z_{0}\right)^{m}$. Set $z=\mathfrak{s}\left(z_{0}\right)$. Now we look at the action $\theta=\operatorname{Ad}(z)$ on $\mathfrak{S}(\tilde{p})$ :

$$
\begin{aligned}
\theta^{m}(\mathfrak{S}(\tilde{p})) & =j\left(u\left(z_{0}^{m}, \tilde{p}\right)\right) \mathfrak{S}\left(\tilde{p} z_{0}^{m}\right) z^{-m} \\
& =j\left(u\left(z_{0}^{m}, \tilde{p}\right) u\left(\tilde{p} z_{0}^{m}, z_{0}^{-m}\right)\right) \mathfrak{S}(\tilde{p}) .
\end{aligned}
$$

Set

$$
\begin{aligned}
v(\tilde{p}, m) & =u\left(z_{0}^{m}, \tilde{p}\right) u\left(\tilde{p} z_{0}^{m}, z_{0}^{-m}\right), \quad \tilde{p} \in Q_{\mathrm{m}}, m \in \mathbf{Z} ; \\
b(\tilde{p}, m, n) & =\theta_{m}(v(\tilde{p}, n)) v(\tilde{p}, m) v(\tilde{p}, m+n)^{-1} .
\end{aligned}
$$

We claim:

$$
b(\tilde{p}, \cdot, \cdot) \in \mathrm{Z}^{2}(\mathrm{Z}, \mathrm{T}) .
$$

The cocycle property is obvious. So we check if $b(\tilde{p}, m, n) \in \mathrm{T}$ :

$$
\begin{gathered}
j(v(\tilde{p}, m+n)) \mathfrak{S}(\tilde{p})=\theta^{m+n}(\mathfrak{S}(\tilde{p}))=\theta^{m}(j(v(\tilde{p}, n)) \mathfrak{S}(\tilde{p})) \\
=j\left(\theta^{m}(v(\tilde{p}, n)) v(\tilde{p}, m)\right) \mathfrak{s}(\tilde{p}) ; \\
j\left(\theta^{m}(v(\tilde{p}, n)) v(\tilde{p}, m) v(\tilde{p}, m+n)^{-1}\right)=1 .
\end{gathered}
$$

thus $b(\tilde{p}, m, n) \in \mathrm{T}$. The triviality $\mathrm{H}^{2}(\mathbf{Z}, \mathrm{T})=\{1\}$ entails the existence of $a(\tilde{p}, \cdot) \in \mathrm{C}^{1}(\mathrm{Z}, \mathrm{T})$ such that $b(\tilde{p}, \cdot, \cdot)=\partial_{\mathrm{Z}} a(\tilde{p}, \cdot)$, i.e.,

$$
\theta_{m}(v(\tilde{p}, n)) v(\tilde{p}, m) v(\tilde{p}, m+n)^{-1}=a(\tilde{p}, m) a(\tilde{p}, n) a(\tilde{p}, m+n)^{-1} .
$$


Setting

$$
w(\tilde{p}, m)=a(\tilde{p}, m)^{-1} v(\tilde{p}, m) \in B
$$

we get

$$
w(\tilde{p}, m+n)=\theta^{m}(w(\tilde{p}, n)) w(\tilde{p}, m)
$$

and

$$
z^{m} \mathfrak{g}(\tilde{p}) z^{-m}=j(w(\tilde{p}, m)) \mathfrak{s}(\tilde{p}) .
$$

Once again the triviality $\mathrm{H}_{\theta}^{1}(\mathrm{Z}, B)=\{1\}$ gives the existence of $a(\tilde{p}) \in B$ so that $w(\tilde{p}, m)=\theta^{m}\left(a(\tilde{p})^{-1}\right) a(\tilde{p})$. Hence we get

$$
\begin{aligned}
z^{m} \mathfrak{S}(\tilde{p}) z^{-m} & =j(w(\tilde{p}, m)) \mathfrak{S}(\tilde{p})=j\left(\theta^{m}\left(a(\tilde{p})^{-1}\right) a(\tilde{p})\right) \mathfrak{s}(\tilde{p}) ; \\
z^{m} j(a(\tilde{p})) \mathfrak{S}(\tilde{p}) z^{-m} & =j(a(\tilde{p})) \mathfrak{S}(\tilde{p}) .
\end{aligned}
$$

Therefore, $z$ and $\dot{\mathfrak{s}}(\tilde{p})=j(a(\tilde{p})) \mathfrak{s}(\tilde{p}), \tilde{p} \in Q_{\mathrm{m}}$, commute.

Proof of Theorem 3.5. i) With the last lemma, we replace the cochain $u \in \mathrm{C}^{2}\left(Q_{\mathrm{m}}, B\right)$ by the cochain $\dot{u} \in \mathrm{C}^{2}\left(Q_{\mathrm{m}}, B\right)$ given by:

$\dot{u}(\tilde{p}, \tilde{q})=a(\tilde{p}) \alpha_{\tilde{p}}(a(\tilde{q})) u(\tilde{p}, \tilde{q}) a(\tilde{p} \tilde{q})^{-1}=\left(u \partial_{Q_{\mathrm{m}}} a\right)(\tilde{p}, \tilde{q}), \quad \tilde{p}, \tilde{q} \in Q_{\mathrm{m}}$,

which still cobounds the cocycle $i_{*}(c) \in \mathrm{Z}^{3}\left(Q_{\mathrm{m}}, B\right)$. Now as

$$
\dot{\mathfrak{H}}_{L}(\tilde{q}, \tilde{r})=j(\dot{u}(\tilde{q}, \tilde{r})) \in L,
$$

$\dot{u}(\tilde{q}, \tilde{r}) \in E=j^{-1}(L) \subset B$ and

$$
\left.\begin{array}{rl}
\dot{u}\left(x z_{0} ; \tilde{q}, \tilde{r}\right) & =\dot{u}(x ; \tilde{q}, \tilde{r}) \dot{u}\left(z_{0} ; \tilde{q}, \tilde{r}\right) ; \\
\partial_{\theta}(\dot{u}(\tilde{q}, \tilde{r})) & =\dot{u}\left(z_{0} ; \tilde{q}, \tilde{r}\right) \in \mathrm{R} / T Z ; \\
\theta^{m}(\dot{u}(\tilde{q}, \tilde{r})) & =\dot{u}\left(z_{0} ; \tilde{q}, \tilde{r}\right)^{m} \dot{u}(\tilde{q}, \tilde{r}) .
\end{array}\right\} \quad x, \tilde{q}, \tilde{r} \in Q_{\mathrm{m}} .
$$

The calculation:

$$
\begin{aligned}
\dot{\mathfrak{n}}_{L}\left(\tilde{q} z_{0}^{m}, \tilde{r} z_{0}^{n}\right) & =\dot{\mathfrak{s}}\left(\tilde{q} z_{0}^{m}\right) \dot{\mathfrak{S}}\left(\tilde{r} z_{0}^{n}\right) \dot{\mathfrak{S}}\left(\tilde{q} \tilde{r} z_{0}^{m+n}\right)^{-1} \\
& =\dot{\mathfrak{s}}(\tilde{q}) z^{m} \dot{\mathfrak{s}}(\tilde{r}) z^{n}\left(\dot{\mathfrak{s}}(\tilde{q} \tilde{r}) z^{m+n}\right)^{-1} \\
& =\dot{\mathfrak{s}}(\tilde{q}) \dot{\mathfrak{s}}(\tilde{r}) \dot{\mathfrak{s}}(\tilde{q} \tilde{r})^{-1} \\
& =\dot{\mathfrak{n}}_{L}(\tilde{q}, \tilde{r}),
\end{aligned}
$$

shows that

i) the cocycle $\dot{\mathfrak{n}}_{L}$ is the pullback $\pi_{\mathrm{m}}^{*}\left(\mathfrak{n}_{L}^{Q}\right)$ of a cocycle $\mathfrak{n}_{L}^{Q} \in \mathrm{Z}_{\alpha}^{2}(Q, L)$;

ii) there exists $f \in \mathrm{C}^{2}\left(Q_{\mathrm{m}}, \mathrm{T}\right)$ such that

$$
\dot{u}(\tilde{p}, \tilde{q})=f(\tilde{p}, \tilde{q}) \mathfrak{S}_{j}\left(\mathfrak{n}_{L}^{Q}(p, q)\right), \quad \tilde{p}, \tilde{q} \in Q_{\mathrm{m}}, p=\pi_{\mathrm{m}}(\tilde{p}), q=\pi_{\mathrm{m}}(\tilde{q}) .
$$


Therefore with $v(p, q)=\mathfrak{s}_{j}\left(\mathfrak{n}_{L}^{Q}(p, q)\right)$ we have

$$
\begin{aligned}
c(\tilde{p}, \tilde{q}, \tilde{r}) & =\left(\partial_{Q_{\mathrm{m}}} \dot{u}\right)(\tilde{p}, \tilde{q}, \tilde{r})=\left(\partial_{Q_{\mathrm{m}}} f\right)(\tilde{p}, \tilde{q}, \tilde{r})\left(\partial_{Q_{\mathrm{m}}} \pi_{\mathrm{m}}^{*}(v)\right)(\tilde{p}, \tilde{q}, \tilde{r}) \\
& =\left(\partial_{Q_{\mathrm{m}}} f\right)(\tilde{p}, \tilde{q}, \tilde{r}) \alpha_{\tilde{p}}(v(q, r)) v(p, q r)\{v(p, q) v(p q, r)\}^{-1} \\
& =\left(\partial_{Q_{\mathrm{m}}} f\right)(\tilde{p}, \tilde{q}, \tilde{r}) \alpha_{\tilde{p}}(v(q, r)) v(p, q r)\{v(p, q) v(p q, r)\}^{-1} \\
& =\left(\partial_{Q_{\mathrm{m}}} f\right)(\tilde{p}, \tilde{q}, \tilde{r}) c_{\mathrm{s}}(\tilde{p}, \tilde{q}, \tilde{r})
\end{aligned}
$$

where

$c_{\mathrm{S}}(\tilde{p}, \tilde{q}, \tilde{r})=\alpha_{\tilde{p}}(v(q, r)) v(p, q r)\{v(p, q) v(p q, r)\}^{-1}=\left(\partial_{Q_{\mathrm{m}}} \pi_{\mathrm{m}}^{*}(v)\right)(\tilde{p}, \tilde{q}, \tilde{r})$.

Now the original cocycle $c$ is cohomologous to $c_{\mathrm{s}}$. We then obtain the following:

$$
\begin{aligned}
c_{\mathrm{s}}\left(\tilde{p} z_{0}^{m}, \tilde{q} z_{0}^{n}, \tilde{r} z_{0}^{\ell}\right) & =\alpha_{\tilde{p}} \circ \theta^{m}(v(q, r)) v(p, q r)\{v(p, q) v(p q, r)\}^{-1} \\
& =\alpha_{\tilde{p}}\left(\theta^{m}(v(q, r)) v(q, r)^{-1}\right) c_{\mathrm{s}}(\tilde{p}, \tilde{q}, \tilde{r}) \\
& =v\left(z_{0} ; q, r\right)^{m} c_{\mathrm{s}}(\tilde{p}, \tilde{q}, \tilde{r}) .
\end{aligned}
$$

Hence $d_{c}(q, r)=v\left(z_{0} ; q, r\right)$ gives the $d$-part of $c_{\mathrm{s}}$.

ii) Suppose that $c \in Z^{3}\left(Q_{\mathrm{m}}, \mathrm{T}\right)$ is standard. Then we have

$$
d_{c}(q, r)=c\left(z_{0}, \tilde{q}, \tilde{r}\right), \quad c\left(z_{0}, \tilde{q}, \tilde{r}\right)=c\left(z_{0} \tilde{p}, \tilde{q}, \tilde{r}\right) \overline{c(\tilde{p}, \tilde{q}, \tilde{r})} .
$$

Therefore we compute for $\tilde{p}, \tilde{q}, \tilde{r} \in Q_{m}$ :

$$
\begin{aligned}
d_{c}(q, r) & \overline{d_{c}(p q, r)} d_{c}(p, q r) \overline{d_{c}(p, q)} \\
\quad & c\left(z_{0}, \tilde{q}, \tilde{r}\right) \overline{c\left(z_{0}, \tilde{p q}, \tilde{r}\right)} c\left(z_{0}, \tilde{p}, \tilde{q r}\right) \overline{c\left(z_{0}, \tilde{p}, \tilde{q}\right)} \\
& =c\left(z_{0}, \tilde{q}, \tilde{r}\right) \overline{c\left(z_{0}, \tilde{p} \tilde{q} z_{0}^{-n(p, q)}, \tilde{r}\right)} c\left(z_{0}, \tilde{p}, \tilde{q} \tilde{r} z_{0}^{-n(q, r)}\right) \overline{c\left(z_{0}, \tilde{p}, \tilde{q}\right)} \\
& =c\left(z_{0}, \tilde{q}, \tilde{r}\right) \overline{c\left(z_{0}, \tilde{p} \tilde{q}, \tilde{r}\right)} c\left(z_{0}, \tilde{p}, \tilde{q} \tilde{r}\right) \overline{c\left(z_{0}, \tilde{p}, \tilde{q}\right)} \\
& =\overline{c(\tilde{p}, \tilde{q}, \tilde{r})} c\left(z_{0} \tilde{p}, \tilde{q}, \tilde{r}\right) \overline{c\left(z_{0}, \tilde{p} \tilde{q}, \tilde{r}\right)} c\left(z_{0}, \tilde{p}, \tilde{q} \tilde{r}\right) \overline{c\left(z_{0}, \tilde{p}, \tilde{q}\right)} \\
& =\partial_{Q_{m}}(\bar{c})\left(z_{0}, \tilde{p}, \tilde{q}, \tilde{r}\right)=1 .
\end{aligned}
$$

Hence we conclude that $d_{c} \in \mathrm{Z}^{2}(Q, \mathrm{~T})$.

iii) Suppose that $c=\partial_{Q_{\mathrm{m}}} f, f \in \mathrm{C}^{2}\left(Q_{\mathrm{m}}, \mathrm{T}\right)$, is standard with $d$-part $d$. We then compute:

$$
\begin{aligned}
& d(q, r)^{m} c(\tilde{p}, \tilde{q}, \tilde{r})=(\partial f)\left(\tilde{p} z_{0}^{m}, \tilde{q} z_{0}^{n}, \tilde{r} z_{0}^{\ell}\right) \\
& \quad=f\left(\tilde{q} z_{0}^{n}, \tilde{r} z_{0}^{\ell}\right) \overline{f\left(\tilde{p} \tilde{q} z_{0}^{m+n}, \tilde{r} z_{0}^{\ell}\right)} f\left(\tilde{p} z_{0}^{m}, \tilde{q} \tilde{r} z_{0}^{n+\ell}\right) \overline{f\left(\tilde{p} z_{0}^{m}, \tilde{q} z_{0}^{n}\right)}
\end{aligned}
$$


Setting $\tilde{p}=1$, we get

$$
\begin{aligned}
d(q, r)^{m} & =(\partial f)\left(z_{0}^{m}, \tilde{q} z_{0}^{n}, \tilde{r} z_{0}^{\ell}\right), \quad m, n, \ell \in \mathbf{Z}, \\
& =f\left(\tilde{q} z_{0}^{n}, \tilde{r} z_{0}^{\ell}\right) \overline{f\left(\tilde{q} z_{0}^{m+n}, \tilde{r} z_{0}^{\ell}\right)} f\left(z_{0}^{m}, \tilde{q} \tilde{r} z_{0}^{n+\ell}\right) \overline{f\left(z_{0}^{m}, \tilde{q} z_{0}^{n}\right)}
\end{aligned}
$$

Setting $\tilde{q}=\tilde{r}=1$, we obtain the cocycle property of $\left.f\right|_{Z^{2}}$ :

$$
1=f\left(z_{0}^{n}, z_{0}^{\ell}\right) \overline{f\left(z_{0}^{m+n}, z_{0}^{\ell}\right)} f\left(z_{0}^{m}, z_{0}^{n+\ell}\right) \overline{f\left(z_{0}^{m}, z_{0}^{n}\right)},
$$

Since $\mathrm{H}^{2}(Z, T)=\{1\}$, there exists $g \in \mathrm{C}^{1}(Z, T)$ such that

$$
f\left(z_{0}^{m}, z_{0}^{n}\right)=\overline{g\left(z_{0}^{m}\right) g\left(z_{0}^{n}\right)} g\left(z_{0}^{m+n}\right) .
$$

Extend $g$ to the entire $Q_{\mathrm{m}}$ and replace $f$ by $\left(\partial_{Q_{\mathrm{m}}} g\right) f$ to get

$$
f\left(z_{0}^{m}, z_{0}^{n}\right)=1, \quad m, n \in \mathbf{Z} .
$$

Setting $\tilde{q}=1, \tilde{r}=1$ and $m=0$ in (3.4), we get

$$
\begin{aligned}
& 1=f\left(z_{0}^{n}, z_{0}^{\ell}\right) \overline{f\left(\tilde{p} z_{0}^{n}, z_{0}^{\ell}\right)} f\left(\tilde{p}, z_{0}^{n+\ell}\right) \overline{f\left(\tilde{p}, z_{0}^{\ell}\right)} ; \\
& f\left(\tilde{p}, z_{0}^{n+\ell}\right)=\left(\left(\theta^{n} \otimes \mathrm{id}\right) f\right)\left(\tilde{p}, z_{0}^{\ell}\right) f\left(\tilde{p}, z_{0}^{\ell}\right) .
\end{aligned}
$$

Hence the cochain: $k \in \mathbf{Z} \mapsto f\left(\cdot, z_{0}^{k}\right) \in B$ is a cocycle, thus the triviality $\mathrm{H}_{\theta}^{1}(Z, B)=\{1\}$ gives the existence of $g \in \mathrm{C}_{\alpha}^{1}\left(Q_{\mathrm{m}}, \mathrm{T}\right)$ such that

$$
f\left(\tilde{p}, z_{0}^{\ell}\right)=g\left(\tilde{p} z_{0}^{\ell}\right) g(\tilde{p})^{-1} .
$$

As a constant multiplication on $g$ does not affect on the above identity, we may and do assume that $g(1)=1$. Observing

$$
\begin{aligned}
1 & =f\left(z_{0}^{n}, z_{0}^{\ell}\right)=g\left(z_{0}^{n+\ell}\right) g\left(z_{0}^{n}\right)^{-1} ; \\
g\left(z_{0}^{n+\ell}\right) & =g\left(z_{0}^{n}\right)=g(1)=1, \quad n, \ell \in Z,
\end{aligned}
$$

we get

$$
f\left(\tilde{p}, z_{0}^{\ell}\right)=g\left(z_{0}^{\ell}\right)^{-1} g\left(\tilde{p} z_{0}^{\ell}\right) g(\tilde{p})^{-1}=\partial_{Q_{\mathrm{m}}} g\left(\tilde{p}, z_{0}^{\ell}\right)^{-1} .
$$

Now with $f^{\prime}=f \partial_{Q_{\mathrm{m}}} g$, we obtain

$$
f^{\prime}\left(\tilde{p}, z_{0}^{n}\right)=1 ; \quad c=\partial_{Q_{\mathrm{m}}} f^{\prime} .
$$

By replacing $f$ by $f^{\prime}$, we may assume $f\left(\tilde{p}, z_{0}^{n}\right)=1$ and $c=\partial_{Q_{\mathrm{m}}} f$. 
Since $f\left(\tilde{p}, z_{0}^{n}\right)=1$, for triplet $\left(\tilde{p}, z_{0}^{n}, \tilde{q}\right)$ in (3.4), we get

$$
\begin{aligned}
& f\left(z_{0}^{n}, \tilde{q}\right) \overline{f\left(\tilde{p} z_{0}^{n}, \tilde{q}\right)} f\left(\tilde{p}, z_{0}^{n} \tilde{q}\right) \overline{f\left(\tilde{p}, z_{0}^{n}\right)}=c\left(\tilde{p}, z_{0}^{n}, \tilde{q}\right)=1 ; \\
& f\left(\tilde{p} z_{0}^{n}, \tilde{q}\right)=f\left(z_{0}^{n}, \tilde{q}\right) f\left(\tilde{p}, z_{0}^{n} \tilde{q}\right)
\end{aligned}
$$

and for triplet $\left(\tilde{p}, \tilde{q}, z_{0}^{n}\right)$ in (3.4), we obtain

$$
\begin{aligned}
& f\left(\tilde{q}, z_{0}^{n}\right) \overline{f\left(\tilde{p} \tilde{q}, z_{0}^{n}\right)} f\left(\tilde{p}, \tilde{q} z_{0}^{n}\right) \overline{f(\tilde{p}, \tilde{q})}=c\left(\tilde{p}, \tilde{q}, z_{0}^{n}\right)=1 ; \\
& f\left(\tilde{p}, \tilde{q} z_{0}^{n}\right)=f(\tilde{p}, \tilde{q})
\end{aligned}
$$

which implies that $f(\tilde{p}, \tilde{q})$ is invariant with respect to $z_{0}^{n}$ in the second variable. For triplet $\left(z_{0}^{m}, z_{0}^{n}, \tilde{q}\right)$ in (3.4), we also get

$$
\begin{aligned}
& f\left(z_{0}^{n}, \tilde{q}\right) \overline{f\left(z_{0}^{m} z_{0}^{n}, \tilde{q}\right)} f\left(z_{0}^{m}, z_{0}^{n} \tilde{q}\right) \overline{f\left(z_{0}^{m}, z_{0}^{n}\right)}=c\left(z_{0}^{m}, z_{0}^{n}, \tilde{q}\right)=1 ; \\
& f\left(z_{0}^{n}, \tilde{q}\right) f\left(z_{0}^{m}, \tilde{q}\right)=f\left(z_{0}^{m+n}, \tilde{q}\right) .
\end{aligned}
$$

We conclude that if $c$ is a coboundary on $Q_{\mathrm{m}}$, then there is a function $f \in$ $\mathrm{C}^{2}\left(Q_{\mathrm{m}}, \mathrm{T}\right)$ with $c=\partial_{Q_{\mathrm{m}}} f$ such that for all $\tilde{p}, \tilde{q} \in Q_{\mathrm{m}}$,

$$
\left\{\begin{array}{l}
f\left(\tilde{p} z_{0}^{n}, \tilde{q} z_{0}^{m}\right)=f\left(z_{0}^{n}, \tilde{q}\right) f(\tilde{p}, \tilde{q}) \\
f\left(z_{0}^{n}, \tilde{q}\right) f\left(z_{0}^{m}, \tilde{q}\right)=f\left(z_{0}^{m+n}, \tilde{q}\right) ; \\
f(\tilde{p}, 1)=f(1, \tilde{q})=1 .
\end{array}\right.
$$

This completes the proof of the theorem.

We apply Theorem 3.5 to the situation:

i) the group $Q$ is the quotient group $G / N$ of a discrete group $G$ by a central normal subgroup $N$;

ii) $\mathrm{m}: G \mapsto \mathrm{R} / T^{\prime} \mathrm{Z}$ is a homomorphism which factors through $Q$, i.e., $\operatorname{Ker}(\mathrm{m}) \supset N$ so that $\mathrm{m}=\mathrm{m} \circ \pi_{\mathrm{m}}$ employing the same notation $\mathrm{m}$ for the homomorphism from $Q$ to $\mathrm{R} / T^{\prime} \mathrm{Z}$ induced from $\mathrm{m} \in \operatorname{Hom}\left(G, \mathrm{R} / T^{\prime} \mathrm{Z}\right)$;

iii) the group $Q_{\mathrm{m}}$ is given by:

$$
Q_{\mathrm{m}}=\left\{\tilde{p}=(p, s) \in Q \times \mathrm{R}: \mathrm{m}(p)=\dot{s}_{T^{\prime}}=s+T^{\prime} \mathrm{Z} \in \mathrm{R} / T^{\prime} \mathrm{Z}\right\},
$$

hence the quotient map $\pi_{\mathrm{m}}$ is precisely the projection map

$$
\pi_{\mathrm{m}}=\operatorname{pr}_{1}: \tilde{p}=(p, s) \in Q_{\mathrm{m}} \mapsto p \in Q
$$

to the first component; 
iv) the distinguished central element $z_{0}$ is given by:

$$
z_{0}=\left(1, T^{\prime}\right) \in Q_{\mathrm{m}}
$$

In this setting, we make the following:

Definition 3.7. The standard coboundaries $\mathrm{B}_{\mathrm{s}}^{3}\left(Q_{\mathrm{m}}, \mathrm{T}\right)$ in $\mathrm{Z}_{\mathrm{s}}^{3}\left(Q_{\mathrm{m}}, \mathrm{T}\right)$ are given by:

$$
\mathrm{B}_{\mathrm{s}}^{3}\left(Q_{\mathrm{m}}, \mathrm{T}\right)=\partial_{Q_{\mathrm{m}}}\left(\pi_{\mathrm{m}}^{*}\left(\mathrm{C}^{2}(Q, \mathrm{~T})\right)\right) \subset \mathrm{B}^{3}\left(Q_{\mathrm{m}}, \mathrm{T}\right) .
$$

The standard third cohomology group $\mathrm{H}_{\mathrm{s}}^{3}\left(Q_{\mathrm{m}}, \mathrm{T}\right)$ is defined to be the quotient group:

$$
\mathrm{H}_{\mathrm{s}}^{3}\left(Q_{\mathrm{m}}, \mathrm{T}\right)=\mathrm{Z}_{\mathrm{s}}^{3}\left(Q_{\mathrm{m}}, \mathrm{T}\right) / \mathrm{B}_{\mathrm{s}}^{3}\left(Q_{\mathrm{m}}, \mathrm{T}\right),
$$

which is a compact abelian group.

The reason for this smaller coboundary group comes from the fact that when we perturb an outer action $\alpha_{g}, g \in G$, on a factor $\mathcal{M}$, we do not allow a perturbation by $\operatorname{Cnt}_{\mathrm{r}}(\mathcal{M})$ but by $\operatorname{Int}(\mathcal{M})$. So even if we consider an outer action of the bigger group $G_{\mathrm{m}}$ on the discrete core $\widetilde{\mathcal{M}}_{d}$, we can not use all of $U(\widetilde{\mathcal{M}})$ but only $U(\mathcal{M})$.

The $d$-part $d_{c}$ of each $c \in \mathrm{Z}_{\mathrm{s}}^{3}\left(Q_{\mathrm{m}}, \mathrm{T}\right)$ is an element of $\mathrm{Z}^{2}(Q, \mathrm{~T})$ and also each $v \in \operatorname{Hom}(N, \mathbf{R} / T Z)$ gives rise to an element of $\mathrm{Z}^{2}(Q, \mathbf{T}):(q, r) \in Q^{2} \mapsto$ $v\left(\mathfrak{n}_{L}(q, r)\right) \in \mathrm{R} / T Z$ under the identification of $\dot{s}_{T} \in \mathrm{R} / T Z$ and $e^{i T^{\prime} s} \in \mathrm{T}, s \in$ R. Hence we define $\mathrm{Z}_{\mathrm{s}}^{3}\left(Q_{\mathrm{m}}, \mathrm{T}\right) *_{3} \operatorname{Hom}_{G}(N, \mathrm{R} / T \mathrm{Z})$ to be the subgroup of $Z_{\mathrm{s}}^{3}\left(Q_{\mathrm{m}}, \mathrm{T}\right) \times \operatorname{Hom}_{G}(N, \mathrm{R} / T \mathrm{Z})$ consisting of all those elements $(c, v)$ such that

$$
d_{c}(q, r)=v\left(\mathfrak{n}_{L}(q, r)\right), \quad q, r \in Q .
$$

Here the fiber product depends on the cocycle $\mathfrak{n}_{L} \in \mathrm{Z}^{2}(Q, L)$ explicitly and therefore on the cross-section $\mathfrak{s}: Q \mapsto G$ of $\pi$.

Theorem 3.8. Fix $0<\lambda<1$ and set

$$
T=-\frac{2 \pi}{\log \lambda}, \quad T^{\prime}=\frac{2 \pi}{T}=-\log \lambda \quad \text { and } \quad X=\mathrm{R} / T^{\prime} \mathrm{Z} .
$$

Let $G$ be a group equipped with a central subgroup $N$. For a homomorphism $\mathrm{m}: G \mapsto X$ such that $\operatorname{Ker}(\mathrm{m}) \supset N$, consider the joint action of $\widetilde{G}=G \times \mathrm{R}$ on $X$ given by:

$$
T_{g, s}(x)=x-\dot{s}_{T^{\prime}}+\mathrm{m}(g) \in X, \quad(g, s) \in \widetilde{G},
$$


and the action $\alpha$ of $\widetilde{G}$ on $\mathcal{C}=L^{\infty}(X)$ given by:

$$
\alpha_{g, s} f(x)=f\left(T_{g, s}^{-1} x\right), \quad x \in X=\mathrm{R} / T^{\prime} Z, \quad f \in \mathcal{C} .
$$

The action of $\mathrm{R}$ alone denoted by $\theta$ is the transitive flow with period $T^{\prime}$ and gives $\mathrm{H}_{\theta}^{1}(\mathrm{R}, A) \cong \mathrm{R} / T \mathrm{Z}$ where $A=U\left(L^{\infty}(X)\right)$. Define $Q=G / N$ and $Q_{\mathrm{m}}=\left\{(p, s) \in Q \times \mathrm{R}: \mathrm{m}(p)=\dot{s}_{T} \in \mathrm{R} / T^{\prime} \mathrm{Z}\right\}$. Define the subgroup $\mathrm{B}_{\mathrm{m}, \mathfrak{\xi}}^{\text {out }}(G, N, \mathrm{~T})$ to be the subgroup of $\mathrm{Z}_{\mathrm{s}}^{3}\left(Q_{\mathrm{m}}, \mathrm{T}\right) *_{\xi} \operatorname{Hom}_{G}(N, \mathrm{R} / T \mathrm{Z})$ consisting of all those elements $(c, v)$ of the form:

$$
c=\partial_{Q_{\mathrm{m}}}\left(\pi_{\mathrm{m}}^{*} f\right) \quad \text { and } \quad v \equiv 1,
$$

for some $f \in \mathrm{C}^{2}(Q, \mathrm{~T})$ and form the quotient group:

$$
\mathrm{H}_{\mathrm{m}, \mathfrak{\xi}}^{\text {out }}(G, N, \mathrm{~T})=\left(\mathrm{Z}_{\mathrm{s}}^{3}\left(Q_{\mathrm{m}}, \mathrm{T}\right) *_{\mathfrak{\xi}} \operatorname{Hom}_{G}(N, \mathrm{R} / T \mathrm{Z})\right) / \mathrm{B}_{\mathrm{m}, \mathfrak{\xi}}^{\text {out }}(G, N, \mathrm{~T})
$$

Then there is a natural isomorphism:

$$
\mathrm{H}_{\alpha, \mathfrak{F}}^{\text {out }}(\widetilde{G}, N, A) \cong \mathrm{H}_{\mathrm{m}, \mathfrak{F}}^{\text {out }}(G, N, \mathrm{~T}) .
$$

The joint action of $\widetilde{Q}=Q \times \mathrm{R}$ on $X=\mathrm{R} / T^{\prime} \mathrm{Z}$ is transitive. But the coboundary group $\mathrm{B}_{\alpha, \mathrm{s}}^{3}(\widetilde{Q}, A)=\partial_{\widetilde{Q}}\left(\mathrm{C}^{2}(Q, A)\right)$ is smaller than the regular coboundary group $\partial_{\widetilde{Q}}\left(C^{2}(\widetilde{Q}, A)\right)$ for the third cohomology group $\mathrm{H}_{\alpha}^{3}(\widetilde{Q}, A)$. So it is not clear whether the straightforward Shapiro machine works or not. We have seen that for the relative cohomology group $\Lambda_{\alpha}(\widetilde{H}, L, M, A)$ the Shapiro machinery works in Proposition 1.11. So we begin by looking at a resolution system $\pi_{G}: H \mapsto G$ with $L=\pi_{G}^{-1}(N)$ and $M=\operatorname{Ker}\left(\pi_{G}\right)$, so that $c \in$ $\mathrm{Z}_{\alpha, \mathfrak{Y}}^{\text {out }}(G, N, A)$ is of the form $c=\delta(\lambda, \mu)$ for some $(\lambda, \mu) \in \mathrm{Z}_{\alpha}(\widetilde{H}, L, M, A)$. Let $E=E(\lambda, \mu) \in \operatorname{Xext}(\widetilde{H}, L, M, A)$ be the corresponding crossed extension equipped with a cross-section $\mathfrak{s}_{j}: L \mapsto E$ such that

$$
\begin{aligned}
\mu(m, n) & =\mathfrak{g}_{j}(m) \mathfrak{g}_{j}(n) \mathfrak{g}_{j}(m n)^{-1}, \quad m, n \in L ; \\
\lambda(m, h, s) & =\alpha_{h, s}\left(\mathfrak{g}_{j}\left(h^{-1} m h\right)\right) \mathfrak{g}_{j}(m)^{-1}, \quad(h, s) \in \widetilde{Q} .
\end{aligned}
$$

Let $\rho$ be the groupoid homomorphism of $\tilde{\mathscr{H}}=X \rtimes \widetilde{H}$ to the stabilizer subgroup $H_{\mathrm{m}}=\left\{(h, s) \in \widetilde{H}: \mathrm{m}(h)=\dot{s}_{T^{\prime}}\right\}$, where the map $\mathrm{m}: H \mapsto \mathrm{R} / T^{\prime} \mathrm{Z}$ is defined to be the pullback $\mathrm{m}=\mathrm{m} \circ \pi_{G}$ of the corresponding map $\mathrm{m}$ of $G$ by $\pi_{G}$. The map $\rho$ is explicitly written in the form:

$$
\rho(\dot{y}, h, s)=\left(h, s-\{\dot{y}-\mathrm{m}(h)+\dot{s}\}_{T^{\prime}}+\{\dot{y}\}_{T^{\prime}}\right) \in H_{\mathrm{m}}, \quad(\dot{y}, h, s) \in \mathscr{H} .
$$


In fact, we have

$$
\begin{aligned}
\rho(\dot{y}, h k, s+t)= & \left(h k, s+t-\{\dot{y}-\mathrm{m}(h k)+\dot{s}+\dot{t}\}_{T^{\prime}}+\{\dot{y}\}_{T^{\prime}}\right) \\
= & \left(h, s-\{\dot{y}-\mathrm{m}(h)+\dot{s}\}_{T^{\prime}}+\{\dot{y}\}_{T^{\prime}}\right) \\
& \quad \times\left(k, t-\{\dot{y}-\mathrm{m}(h k)+\dot{s}+\dot{t}\}_{T^{\prime}}+\{\dot{y}-\mathrm{m}(h)+\dot{s}\}_{T^{\prime}}\right) \\
= & \rho(\dot{y}, h, s) \rho(\dot{y} \tilde{h}, k, t) \quad \text { with } \quad \tilde{h}=(h, s) .
\end{aligned}
$$

LEMMA 3.9. If $\rho$ is a homomorphism of $\tilde{\mathscr{H}}$ to a group $K$, then for any $c \in \mathrm{Z}^{n}(K, \mathrm{~T})$, the pullback $\rho^{*}(c)$ defined by:

$$
\rho^{*}(c)\left(\dot{x} ; \tilde{h}_{1}, \ldots, \tilde{h}_{n}\right)=c\left(\rho\left(\dot{x}, \tilde{h}_{1}\right), \rho\left(\dot{x} \tilde{h}_{1}, \tilde{h}_{2}\right), \ldots, \rho\left(\dot{x} \tilde{h}_{1} \ldots \tilde{h}_{n-1}, \tilde{h}_{n}\right)\right)
$$

is an element of $\mathrm{Z}_{\alpha}^{n}(\tilde{\mathscr{H}}, \mathrm{T})$ and if $f \in \mathrm{C}^{n-1}(K, \mathrm{~T})$, then

$$
\partial_{\widetilde{H}} \rho^{*}(f)=\rho^{*}\left(\partial_{K} f\right)
$$

where $\rho^{*}(f) \in \mathrm{C}^{n}(\tilde{\mathscr{H}}, \mathrm{T})$ is given by

$$
\begin{aligned}
& \left(\rho^{*} f\right)\left(\dot{x} ; \tilde{h}_{1}, \ldots, \tilde{h}_{n-1}\right) \\
& \quad=f\left(\rho\left(\dot{x}, \tilde{h}_{1}\right), \rho\left(\dot{x} \tilde{h}_{1}, \tilde{h}_{2}\right), \ldots, \rho\left(\dot{x} \tilde{h}_{1} \cdots \tilde{h}_{n-2}, \tilde{h}_{n-1}\right)\right) .
\end{aligned}
$$

Hence $\rho^{*}$ gives a homomorphism of $\mathrm{H}^{n}(K, \mathrm{~T})$ to $\mathrm{H}_{\alpha}^{n}(\tilde{\mathscr{H}}, \mathrm{T})$.

This follows from a direct calculation. We leave it to the reader.

Proof of Theorem 3.8. First, since $\rho(\dot{y}, \tilde{h})=\tilde{h}$ for every $\tilde{h} \in H_{\mathrm{m}}$, we have $i^{*} \circ \rho^{*}=\left.\mathrm{id}\right|_{\mathrm{Z}^{3}\left(H_{\mathrm{m}}, \mathrm{T}\right)}$ where $i$ is the embedding map $i_{\{0\}}$ in Proposition 1.8. Next, choose $(c, v) \in \mathrm{Z}_{\alpha}^{\text {out }}(\widetilde{Q}, A)$ and assume that the system $\pi_{G}: H \mapsto G$ gives a resolution of $\partial(c, v) \in \mathrm{Z}^{3}(G, \mathrm{~T})$ by $(\lambda, \mu) \in \mathrm{Z}_{\alpha}(\widetilde{H}, L, M, A)$ so that $([c], v)=\delta([\lambda, \mu])$ by the modified HJR-map:

$$
\delta: \Lambda_{\alpha}(\widetilde{H}, L, M, A) \mapsto \mathrm{H}_{\alpha}^{\text {out }}(G, N, A) .
$$

This means that if $\mathfrak{S}_{j}$ is the cross-section of the crossed extension:

$$
E=E(\lambda, \mu) \in \operatorname{Xext}_{\alpha}(\tilde{H}, L, M, A)
$$

associated with $(\lambda, \mu)$, the cocycle $(c, \nu)$ is given by:

$$
\begin{aligned}
c(\tilde{p}, \tilde{q}, \tilde{r}) & =\alpha_{\tilde{p}}\left(\mathfrak{S}_{j}\left(\mathfrak{n}_{L}(q, r)\right)\right) \mathfrak{S}_{j}\left(\mathfrak{n}_{L}(p, q r)\right)\left\{\mathfrak{S}_{j}\left(\mathfrak{n}_{L}(p, q) \mathfrak{S}_{j}\left(\mathfrak{n}_{L}(p q, r)\right)\right\}^{-1} ;\right. \\
\nu(n) & =\left[\lambda\left(\mathfrak{F}_{H}(n) ;(1, \cdot)\right)\right] \in \mathrm{H}_{\theta}^{1}(\mathrm{R}, A)=\mathrm{R} / T \mathrm{Z},
\end{aligned}
$$


for $\tilde{p}=(p, s), \tilde{q}=(q, r), \tilde{r}=(r, u) \in \widetilde{Q}$ and $n \in N$ where $\mathfrak{S}_{H}$ is a crosssection of the quotient map $\pi_{G}$. By Proposition 1.11, with $\left(\lambda_{0}, \mu_{0}\right)=i(\lambda, \mu) \in$ $\mathrm{Z}\left(Q_{\mathrm{m}}, L, M, \mathrm{~T}\right)$ we have

$$
(\lambda, \mu) \equiv \rho^{*}\left(\lambda_{0}, \mu_{0}\right) \bmod \mathrm{B}(\widetilde{Q}, L, M, A) .
$$

Therefore we may replace $(\lambda, \mu)$ by $\rho^{*}\left(\lambda_{0}, \mu_{0}\right)$, i.e., we may assume that

$$
\begin{aligned}
& \mu(\dot{y} ; m, n)=\mu_{0}(m, n) \in \mathrm{T}, \quad m, n \in L ; \\
& \lambda(\dot{y} ; m, \tilde{h})=\lambda_{0}(m, \rho(\dot{y}, \tilde{h})), \quad m \in L, \quad \tilde{h} \in \tilde{H}, \quad \dot{y} \in X=\mathrm{R} / T^{\prime} Z .
\end{aligned}
$$

With $E_{0}=E\left(\lambda_{0}, \mu_{0}\right) \in \operatorname{Xext}\left(H_{\mathrm{m}}, L, M, \mathrm{~T}\right)$, we have $E=\rho^{*}\left(E_{0}\right)$, i.e.,

$$
E=A \times E_{0} /\{(a, \bar{a}): a \in \mathrm{T}\}
$$

admits a cross-section $\mathfrak{S}_{j}: m \in L \mapsto\left[\mathfrak{S}_{0}(m)\right] \in E$ such that

$$
\begin{aligned}
\mathfrak{S}_{j}(m) \mathfrak{S}_{j}(n) & =\mu(m, n) \mathfrak{S}_{j}(m n) ; \\
\alpha_{\tilde{h}}\left(\mathfrak{S}_{j}\left(h^{-1} m h\right)\right)(\dot{y}) & =\lambda(\dot{y} ; m, \tilde{h}) \mathfrak{S}_{j}(m)(\dot{y})=\alpha_{\rho(\dot{y}, \tilde{h})}^{0}\left(\mathfrak{S}_{0}\left(h^{-1} m h\right)\right) .
\end{aligned}
$$

Now let us compute, based on the fact that the $L$-valued cocycle $\mathfrak{n}_{L}$ does not depend on the R-variables:

$$
\begin{aligned}
\left(\rho^{*} c^{0}\right) & (\tilde{p}, \tilde{q}, \tilde{r} ; \dot{y}) \\
= & c^{0}(\rho(\dot{y}, \tilde{p}), \rho(\dot{y} \tilde{p}, \tilde{q}), \rho(\dot{y} \tilde{p} \tilde{q}, \tilde{r})) \\
= & \alpha_{\rho(\dot{y}, \tilde{p})}^{0}\left(\mathfrak { g } _ { 0 } ( \mathfrak { n } _ { L } ( \rho ( \dot { y } \tilde { p } , \tilde { q } ) , \rho ( \dot { y } \tilde { p } \tilde { q } , \tilde { r } ) ) ) \mathfrak { s } _ { 0 } \left(\mathfrak{n}_{L}(\rho(\dot{y}, \tilde{p}), \rho(\dot{y} \tilde{p}, \tilde{q} \tilde{r}))\right.\right. \\
& \quad \times\left\{\mathfrak{g}_{0}\left(\mathfrak{n}_{L}(\rho(\dot{y}, \tilde{p}), \rho(\dot{y} \tilde{p}, \tilde{q}))\right) \mathfrak{s}_{0}\left(\mathfrak{n}_{L}(\rho(\dot{y}, \tilde{p} \tilde{q}), \rho(\dot{y} \tilde{p} \tilde{q}, \tilde{r}))\right)\right\}^{-1} \\
= & \left(\alpha_{\tilde{p}}\left(\mathfrak{g}_{j}\left(\mathfrak{n}_{L}(q, r)\right)\right) \mathfrak{g}_{j}\left(\mathfrak{n}_{L}(p, q r)\right)\left\{\mathfrak{g}_{j}\left(\mathfrak{n}_{L}(p, q)\right) \mathfrak{g}_{j}\left(\mathfrak{n}_{L}(p q, r)\right)\right\}^{-1}\right)(\dot{y}) \\
= & c(\tilde{p}, \tilde{q}, \tilde{r} ; \dot{y}) .
\end{aligned}
$$

Suppose that $c^{0} \in \mathrm{B}_{\mathrm{s}}^{3}\left(Q_{\mathrm{m}}, \mathrm{T}\right)$, i.e., for some $f \in \mathrm{C}^{2}(Q, \mathrm{~T})$ we have

$$
c^{0}(\tilde{p}, \tilde{q}, \tilde{r})=f(q, r) \overline{f(p q, r)} f(p, q r) \overline{f(p, q)} ;
$$


Then we have for $c=\rho^{*} c^{0}$ :

$$
\begin{aligned}
\left(\rho^{*} c^{0}\right)(\tilde{p}, \tilde{q}, \tilde{r} ; \dot{y})=f & \left(\pi_{\mathrm{m}}(\rho(\dot{y} \tilde{p}, \tilde{q})), \pi_{\mathrm{m}}(\rho(\dot{y} \tilde{p} \tilde{q}, \tilde{r}))\right) \\
& \times \overline{f\left(\pi_{\mathrm{m}}(\rho(\dot{y}, \tilde{p})) \pi_{\mathrm{m}}(\rho(\dot{y}, \tilde{p})), \pi_{\mathrm{m}}(\rho(\dot{y} \tilde{p}, \tilde{q} \tilde{r}))\right)} \\
& \times f\left(\pi_{\mathrm{m}}(\rho(\dot{y}, \tilde{p})), \pi_{\mathrm{m}}(\rho(\dot{y} \tilde{p}, \tilde{q}) \rho(\dot{y} \tilde{p} \tilde{q}, \tilde{r}))\right) \\
& \times \overline{f\left(\pi_{\mathrm{m}}(\rho(\dot{y}, \tilde{p})), \pi_{\mathrm{m}}(\rho(\dot{y} \tilde{p}, \tilde{q}))\right)} \\
= & f(q, r) \overline{f(p q, r)} f(p, q r) \overline{f(p, q)} \\
=\partial_{\tilde{Q}} & \left(\operatorname{pr}_{1}^{*} f\right)(\tilde{p}, \tilde{q}, \tilde{r} ; \dot{y}),
\end{aligned}
$$

where $\mathrm{pr}_{1}$ is the projection map of $\widetilde{Q}$ to the first component $Q$, so that $\rho^{*} c^{0} \in$ $\partial_{\widetilde{Q}}\left(\mathrm{C}^{2}(Q, \mathrm{~T})\right) \subset \partial_{\widetilde{Q}}\left(\mathrm{C}^{2}(Q, A)\right)$. If $\rho^{*} c^{0} \in \partial_{\widetilde{Q}}\left(\mathrm{C}^{2}(Q, A)\right)$, i.e., if there exists $f \in \mathrm{C}^{2}(Q, A)$ such that $\rho^{*} c^{0}=\partial_{\tilde{Q}} f$, then for each $\tilde{p}, \tilde{q}, \tilde{r} \in Q_{\mathrm{m}}$ we have

$$
\begin{aligned}
c(\tilde{p}, \tilde{q}, \tilde{r} ; \dot{y}) & =f(q, r ; \dot{y}+\mathrm{m}(p)-\dot{s}) f(p, q r ; \dot{y}) \overline{f(p, q ; \dot{y}) f(p q, r ; \dot{y})} \\
& =f(q, r ; \dot{y}) f(p, q r ; \dot{y}) \overline{f(p, q ; \dot{y}) f(p q, r ; \dot{y})} .
\end{aligned}
$$

Hence we get

$$
c^{0}(\tilde{p}, \tilde{q}, \tilde{r})=c(\tilde{p}, \tilde{q}, \tilde{r} ; 0)=\left(\partial_{Q_{\mathrm{m}}} \pi_{\mathrm{m}}^{*}\left(f_{0}\right)\right)(\tilde{p}, \tilde{q}, \tilde{r})
$$

where $f_{0}(p, q)=f(p, q ; 0)$. Thus we conclude $c_{0} \in \partial_{Q_{\mathrm{m}}}\left(\pi_{\mathrm{m}}^{*}\left(\mathrm{C}^{2}(Q, \mathrm{~T})\right)\right)$. Consequently, we get

$$
\mathrm{H}_{\alpha, \mathrm{s}}^{3}(\widetilde{Q}, A) \cong \mathrm{H}_{\mathrm{s}}^{3}\left(Q_{\mathrm{m}}, \mathrm{T}\right) .
$$

We want to compare the $d$-part $d_{c}$ of $c$ and the $d$-part $d_{c}^{0}=d_{c^{0}}$ of $c^{0}$.

In terms of $c$ and $c^{0}, d_{c}$ and $d_{c}^{0}$ are given by the following:

$$
\begin{aligned}
d_{c}(s ; q, r) & =c((1, s),(q, 0),(r, 0)) \\
& =\theta_{s}\left(\mathfrak{g}_{j}\left(\mathfrak{n}_{L}(q, r)\right) \mathfrak{s}_{j}\left(\mathfrak{n}_{L}(1, q r)\right)\left\{\mathfrak{g}_{j}\left(\mathfrak{n}_{L}(1, q)\right) \mathfrak{s}_{j}\left(\mathfrak{n}_{L}(q, r)\right)\right\}^{-1}\right. \\
& =\theta_{s}\left(\mathfrak{g}_{j}\left(\mathfrak{n}_{L}(q, r)\right) \mathfrak{s}_{j}\left(\mathfrak{n}_{L}(q, r)\right)^{-1}\right. \\
& =\lambda\left(\mathfrak{n}_{L}(q, r), s\right) ; \\
d_{c}(s ; q, r ; \dot{y}) & =\lambda\left(\dot{y} ; \mathfrak{n}_{L}(q, r), s\right)=\lambda_{0}\left(\mathfrak{n}_{L}(q, r), \rho(\dot{y}, 1, s)\right) \\
& =\lambda_{0}\left(\mathfrak{n}_{L}(q, r), s-\{\dot{y}+\dot{s}\}_{T^{\prime}}+\{\dot{y}\}_{T^{\prime}}\right) \\
& =\left\langle v\left(\mathfrak{n}_{N}(q, r)\right),\left[\frac{y+s}{T^{\prime}}\right]-\left[\frac{y}{T^{\prime}}\right]\right\rangle \\
& =\exp \left(\mathrm{i} T^{\prime}\left\{v\left(\mathfrak{n}_{N}(q, r)\right)\right\}_{T}\left(\left[\frac{y}{T^{\prime}}\right]-\left[\frac{y+s}{T^{\prime}}\right]\right)\right) ;
\end{aligned}
$$




$$
\begin{aligned}
d_{c}^{0}(q, r) & =c\left(\left(1, T^{\prime}\right),(q, 0),(r, 0)\right) \\
& =\theta_{T^{\prime}}\left(\mathfrak{S}_{j}\left(\mathfrak{n}_{L}(q, r)\right)\right) \mathfrak{S}_{j}\left(\mathfrak{n}_{L}(q, r)\right)^{-1}=\lambda_{0}\left(\mathfrak{n}_{L}(q, r), z_{0}\right) \\
& =\dot{v}\left(\mathfrak{n}_{L}(q, r)\right)=\exp \left(-\mathrm{i} T^{\prime}\left\{v\left(\mathfrak{n}_{L}(q, r)\right)\right\}_{T}\right) \\
& =d_{c}\left(T^{\prime} ; q, r ; 0\right)
\end{aligned}
$$

where the duality pairing of $\mathrm{R} / T \mathrm{Z}$ and its dual $\mathrm{Z}$ will be denoted by

$$
\langle\dot{s}, m\rangle=e^{\mathrm{i} T^{\prime} m\{\dot{s}\}_{T}} \quad \text { for } \quad m \in \mathbf{Z}
$$

and $\dot{s} \in \mathrm{R} / T \mathrm{Z}$ and we write $\dot{v}(m), m \in N$, for $e^{\mathrm{i} T^{\prime}\{v(m)\}_{T}}$ for short.

Hence $(c, v)$ is in $\mathrm{Z}_{\alpha, \mathrm{s}}^{3}(\widetilde{Q}, A) *_{3} \operatorname{Hom}_{G}(N, \mathrm{R} / T \mathrm{Z})$ if and only if $\left(c^{0}, v\right)$ is in $\mathrm{Z}_{\mathrm{s}}^{3}\left(Q_{\mathrm{m}}, \mathrm{T}\right) *_{\xi} \operatorname{Hom}_{G}(N, \mathrm{R} / T \mathrm{Z})$.

Now we suppose $(c, v) \in \mathrm{B}_{\alpha, \mathfrak{g}}^{\text {out }}(G, N, A)$ i.e., there exists $f \in \mathrm{C}_{\alpha}^{2}(Q, A)$ such that for each $\tilde{p}=(p, s), \tilde{q}=(q, t), \tilde{r}=(r, u) \in \widetilde{Q}$, we have

$$
\begin{aligned}
c(\tilde{p}, \tilde{q}, \tilde{r} ; \dot{y}) & =f(q, r ; \dot{y} \tilde{p}) f(p, q r ; \dot{y}) \overline{f(p, q ; \dot{y}) f(p q, r ; \dot{y})}^{-1} \\
d_{c}(s, q, r ; \dot{y}) & =\overline{f(q, r ; \dot{y})} f(q, r ; \dot{y}-\dot{s}) .
\end{aligned}
$$

Then we have, for each $\tilde{p}, \tilde{q}, \tilde{r} \in Q_{\mathrm{m}}$,

$$
\begin{aligned}
c^{0}(\tilde{p}, \tilde{q}, \tilde{r}) & =c(\tilde{p}, \tilde{q}, \tilde{r} ; 0) \\
& =f(q, r ; 0) f(p, q r ; 0) \overline{f(p, q ; 0) f(p q, r ; 0)} \\
d_{c}^{0}(q, r) & =\overline{f(q, r ; 0)} f(q, r ; 0)=1 .
\end{aligned}
$$

Thus we get $\left(c^{0}, v\right) \in \mathrm{B}_{\mathrm{m}, \mathfrak{s}}^{\text {out }}(G, N, \mathrm{~T})$. Conversely, suppose $\left(c^{0}, v\right) \in$ $\mathrm{B}_{\mathrm{m}, \mathfrak{S}}^{\text {out }}\left(G, N\right.$, T), i.e., $v \equiv 1$ and for some $f \in \mathrm{C}^{2}(Q, \mathrm{~T})$,

$$
c^{0}(\tilde{p}, \tilde{q}, \tilde{r})=f(q, r) \overline{f(p q, r)} f(p, q r) \overline{f(p, q)}, \quad \tilde{p}, \tilde{q}, \tilde{r} \in Q_{\mathrm{m}} .
$$

Since we have seen already that $c=\rho^{*} c^{0}$ is cobounded by $\operatorname{pr}_{1}^{*} f$, we have $(c, v) \in \mathrm{B}_{\alpha, \mathfrak{g}}^{\text {out }}(G, N, A)$. This completes the proof.

\section{The Map d}

We now want to identify the map

$$
\partial: \mathrm{H}_{\alpha, \mathfrak{S}}^{\text {out }}(G, N, A) \cong \mathrm{H}_{\mathrm{m}, \mathfrak{S}}^{\text {out }}(G, N, \mathrm{~T}) \longrightarrow \mathrm{Z}^{3}(G, \mathrm{~T})
$$

of [17, Theorem 2.7] in terms of $\mathrm{H}_{\mathrm{m}, \mathfrak{G}}^{\text {out }}(G, N, \mathrm{~T})$. To this end, we need notations to shorten mathematical expressions. We use $\mathfrak{n}_{Z}(p, q)$ for the Z-valued two cocycle $\eta_{T^{\prime}}(\mathrm{m}(p), \mathrm{m}(q)), p, q \in Q$. We also use $\mathfrak{n}_{Z}(g, h)$ for $\mathfrak{n}_{Z}(\pi(g), \pi(h))$ 
omitting the map $\pi$. Also the element $z_{0}=\left(1, T^{\prime}\right)$ appears both in $G_{\mathrm{m}}$ and $Q_{\mathrm{m}}$. So we have

$$
\bar{p} \bar{q}=z_{0}^{\mathfrak{n}_{Z}(p, q)} \overline{p q} ; \quad \bar{g} \bar{h}=z_{0}^{\mathfrak{n}_{Z}(g, h)} \overline{g h},
$$

where $\bar{g}=\left(g,\{\mathrm{~m}(g)\}_{T^{\prime}}\right) \in G_{\mathrm{m}}, g \in G$ and $\bar{p}=\left(p,\{\mathrm{~m}(p)\}_{T^{\prime}}\right) \in Q_{\mathrm{m}}$.

Lemma 3.10. Fix $(c, v) \in \mathrm{Z}_{\mathrm{s}}^{3}\left(Q_{\mathrm{m}}, \mathrm{T}\right) *_{\xi} \operatorname{Hom}_{G}(N, \mathrm{R} / T \mathrm{Z})$. With

$$
\mathrm{n}_{N}(g)=\mathfrak{s}(\pi(g)) g^{-1} \in N, \quad g \in G,
$$

and

$$
\dot{v}(m)=e^{\mathrm{i} T^{\prime}\{v(m)\}_{T}} \in \mathbf{T}, \quad m \in L,
$$

set

$$
c_{G}(g, h, k)=\dot{v}\left(\mathrm{n}_{N}(k)\right)^{-\mathfrak{n}_{Z}(g, h)} c(\bar{p}, \bar{q}, \bar{r}), \quad g, h, k \in G,
$$

where $p=\pi(g), q=\pi(h), r=\pi(k)$. Then $c_{G} \in \mathrm{Z}^{3}(G, \mathrm{~T})$. The map:

$$
([c], v) \in \mathrm{H}_{\mathrm{m}, \mathfrak{S}}^{\mathrm{out}}(G, N, \mathrm{~T}) \longrightarrow\left[c_{G}\right] \in \mathrm{H}^{3}(G, \mathrm{~T})
$$

is precisely the map $\partial$ of [17, Theorem 2.7].

Proof. Since $\mathrm{n}_{N}(g) g=\dot{\mathfrak{s}}(\pi(g)), g \in G$, where $\mathfrak{g}: Q \mapsto G$, we have for each pair $g, h \in G$ :

$$
\begin{aligned}
\mathrm{n}_{N}(\pi(g), \pi(h)) & =\mathfrak{S}(\pi(g)) \mathfrak{S}(\pi(h)) \mathfrak{S}(\pi(g h))^{-1} \\
& =\mathrm{n}_{N}(g) g \mathrm{n}_{N}(h) h\left\{\mathrm{n}_{N}(g h) g h\right\}^{-1} \\
& =\mathrm{n}_{N}(g) g \mathrm{n}_{N}(h) g^{-1} \mathrm{n}_{N}(g h)^{-1} .
\end{aligned}
$$

We compute for $g, h, k, \ell \in G$ with $p=\pi(g), q=\pi(h), r=\pi(k)$ and $s=\pi(\ell)$

$$
\begin{aligned}
c(\bar{q}, \bar{r}, \bar{s}) & \overline{c(\overline{p q}, \bar{r}, \bar{s})} c(\bar{p}, \overline{q r}, \bar{s}) \overline{c(\bar{p}, \bar{q}, \overline{r s})} c(\bar{p}, \bar{q}, \bar{r}) \\
= & c(\bar{q}, \bar{r}, \bar{s}) c \overline{c\left(z_{0}^{-\mathfrak{n}_{Z}(p, q)} \bar{p} \bar{q}, \bar{r}, \bar{s}\right)} c\left(\bar{p}, z_{0}^{-\mathfrak{n}_{Z}(q, r)} \bar{q} \bar{r}, \bar{s}\right) \\
& \quad \times \overline{c\left(\bar{p}, \bar{q}, z_{0}^{-\mathfrak{n}_{Z}(r, s)} \bar{r} \bar{s}\right)} c(\bar{p}, \bar{q}, \bar{r}) \\
= & d_{c}(\pi(k), \pi(\ell))^{\mathfrak{n}_{Z}(g, h)}=\left\langle v\left(\mathfrak{n}_{N}(\pi(k), \pi(\ell))\right), \mathfrak{n}_{Z}(g, h)\right\rangle \\
= & \dot{v}\left(\mathrm{n}_{N}(k) k \mathrm{n}_{N}(\ell) k^{-1} \mathrm{n}_{N}(k \ell)^{-1}\right)^{\mathfrak{n}_{Z}(g, h)}
\end{aligned}
$$


and proceed to obtain the calculation:

$$
\begin{aligned}
c_{G}(h, k, \ell) & \overline{c_{G}(g h, k, \ell)} c_{G}(g, h k, \ell) \overline{c_{G}(g, h, k \ell)} c_{G}(g, h, k) \\
= & \dot{v}\left(\mathrm{n}_{N}(\ell)\right)^{-\mathfrak{n}_{Z}(h, k)} c(\bar{q}, \bar{r}, \bar{s}) \overline{\dot{v}\left(\mathrm{n}_{N}(\ell)\right)^{-\mathfrak{n}_{Z}(g h, k)} c(\overline{p q}, \bar{r}, \bar{s})} \\
& \times \dot{v}\left(\mathrm{n}_{N}(\ell)\right)^{-\mathfrak{n}_{Z}(g, h k)} c(\bar{p}, \overline{q r}, \bar{s}) \\
\quad \times & \overline{\dot{v}\left(\mathrm{n}_{N}(k \ell)\right)^{-\mathfrak{n}_{Z}(g, h)} c(\bar{p}, \bar{q}, \overline{r s})} \\
= & \quad \dot{v}\left(\mathrm{n}_{N}(k)\right)^{-\mathfrak{n}_{Z}(g, h)} c(\bar{p}, \bar{q}, \bar{s}) \\
= & \left(\mathrm{n}_{N}(k) k \mathrm{n}_{N}(\ell) k^{-1} \mathrm{n}_{N}(k \ell)^{-1}\right)^{\mathfrak{n}_{Z}(g, h)} \\
& \times \dot{v}\left(\mathrm{n}_{N}(\ell)\right)^{-\mathfrak{n}_{Z}(h, k)} \dot{v}\left(\mathrm{n}_{N}(\ell)\right)^{\mathfrak{n}_{Z}(g h, k)} \\
& \times \dot{v}\left(\mathrm{n}_{N}(\ell)\right)^{-\mathfrak{n}_{Z}(g, h k)} \dot{v}\left(\mathrm{n}_{N}(k \ell)\right)^{\mathfrak{n}_{Z}(g, h)} \\
= & \times \dot{v}\left(\mathrm{n}_{N}(k)\right)^{-\mathfrak{n}_{Z}(g, h)} \\
=1 &
\end{aligned}
$$

Hence $c_{G}$ belongs to $\mathrm{Z}^{3}(G, \mathrm{~T})$.

Since

$$
\begin{aligned}
\zeta_{v}(t, n)(\dot{x})=c(v(n), t, \dot{x})=\dot{v}(n)^{\left(\left[\frac{x+t}{T^{\prime}}\right]-\left[\frac{x}{T^{\prime}}\right]\right)} \\
=\left\langle\dot{v}(n),\left[\frac{x+t}{T^{\prime}}\right]-\left[\frac{x}{T^{\prime}}\right]\right\rangle, \quad t, x \in \mathrm{R}, n \in N,
\end{aligned}
$$

we compute the element $a$ appeared in (2.23) in [17] as follows;

$$
\begin{aligned}
\left(\theta_{t}(a(g, h)) a(g, h)^{*}\right)(\dot{x}) & \\
=\zeta_{v}( & \left.; \mathfrak{n}_{N}(\pi(g), \pi(h))\right)(\dot{x}) \\
& \times\left\{\zeta_{v}\left(t ; \mathrm{n}_{N}(g)\right)(\dot{x}) \alpha_{g}\left(\zeta_{v}\left(t ; \mathrm{n}_{N}(h)\right)\right)(\dot{x}) \zeta_{v}\left(t ; \mathrm{n}_{N}(g h)\right)^{*}(\dot{x})\right\}^{*} \\
=\langle\dot{v}( & \left.\left.\mathfrak{n}_{N}(\pi(g), \pi(h))\right),\left[\frac{x+t}{T^{\prime}}\right]-\left[\frac{x}{T^{\prime}}\right]\right\rangle \\
\times & \left\{\left\langle\dot{v}\left(\mathrm{n}_{N}(g)\right),\left[\frac{x+t}{T^{\prime}}\right]-\left[\frac{x}{T^{\prime}}\right]\right\rangle\right. \\
\times & \left\langle\dot{v}\left(\mathrm{n}_{N}(h)\right),\left[\frac{x-\{\mathrm{m}(g)\}_{T^{\prime}}+t}{T^{\prime}}\right]-\left[\frac{x-\{\mathrm{m}(g)\}_{T^{\prime}}}{T^{\prime}}\right]\right\rangle \\
\times & \left.\left\langle\dot{v}\left(\mathrm{n}_{N}(g h)\right),\left[\frac{x}{T^{\prime}}\right]-\left[\frac{x+t}{T^{\prime}}\right]\right\rangle\right\}^{*}
\end{aligned}
$$


since $\mathfrak{n}_{N}(\pi(g), \pi(h))=\mathrm{n}_{N}(g) g \mathrm{n}_{N}(h) g^{-1} \mathrm{n}_{N}(g h)^{-1}$ and $v$ is $G$-invariant,

$$
\begin{aligned}
& =\dot{v}\left(\mathrm{n}_{N}(h)\right)\left(\left[\frac{x-\{\mathrm{m}(g)\}_{T^{\prime}}}{T^{\prime}}\right]-\left[\frac{x+t-\{\mathrm{m}(g)\}_{T^{\prime}}}{T^{\prime}}\right]\right) \dot{v}\left(\mathrm{n}_{N}(h)\right)\left(\left[\frac{x+t}{T^{\prime}}\right]-\left[\frac{x}{T^{\prime}}\right]\right) \\
& =\dot{v}\left(\mathrm{n}_{N}(h)\right)^{\left(\left[\frac{x+t}{T^{\prime}}\right]-\left[\frac{x-\{\mathrm{m}(g)\}_{T^{\prime}+t}}{T^{\prime}}\right]\right)} \dot{v}\left(\mathrm{n}_{N}(h)\right)\left(\left[\frac{x-\{\mathrm{m}(g)\}_{T^{\prime}}}{T^{\prime}}\right]-\left[\frac{x}{T^{\prime}}\right]\right),
\end{aligned}
$$

which shows that the cochain $a$ ought to be of the following form:

$$
a(g, h)(x)=\dot{v}\left(\mathrm{n}_{N}(h)\right)\left(\left[\frac{x}{T^{\prime}}\right]-\left[\frac{x-\{\mathrm{m}(g)\}_{T^{\prime}}}{T^{\prime}}\right]\right) .
$$

Now we examine the proof of [17, Theorem 2.7], in particular the proof of Lemma 2.11. The split property of the exact sequence:

$$
1 \longrightarrow \mathrm{B}_{\theta}^{1} \longrightarrow \mathrm{Z}_{\theta}^{1} \underset{\mathfrak{s}_{\mathrm{Z}}}{\rightleftarrows} \mathrm{H}_{\theta}^{1}=\mathrm{R} / T \mathrm{Z} \longrightarrow 1
$$

allows us to choose $f=1$ in [17, (2.20)].

We compute the map $\partial$ as follows:

$$
\begin{aligned}
&(\partial c)(g, h, k)=c(\pi(g), \pi(h), \pi(k))(x)\left(\partial_{G} a^{*}\right)(g, h, k)(x) \\
&=c(\pi(g), \pi(h), \pi(k))(x) \dot{v}\left(\mathrm{n}_{N}(k)\right)\left(\left[\frac{x-\{\mathrm{m}(g)\}_{T^{\prime}}-\{\mathrm{m}(h)\}_{T^{\prime}}}{T^{\prime}}\right]-\left[\frac{x-\{\mathrm{m}(g)\}_{T^{\prime}}}{T^{\prime}}\right]\right) \\
& \quad \times \dot{v}\left(\mathrm{n}_{N}(h k)\right)\left(\left[\frac{x-\{\mathrm{m}(g)\}_{T^{\prime}}}{T^{\prime}}\right]-\left[\frac{x}{T^{\prime}}\right]\right) \dot{v}\left(\mathrm{n}_{N}(h)\right)\left(\left[\frac{x}{T^{\prime}}\right]-\left[\frac{x-\{\mathrm{m}(g)\}_{T^{\prime}}}{T^{\prime}}\right]\right) \\
& \quad \times \dot{v}\left(\mathrm{n}_{N}(k)\right)\left(\left[\frac{x}{T^{\prime}}\right]-\left[\frac{x-\{\mathrm{m}(g h)\}_{T^{\prime}}}{T^{\prime}}\right]\right) \\
&=c(\pi(g), \pi(h), \pi(k))(x) \exp \left(-\mathrm{i} v\left(\mathrm{n}_{N}(k)\right) \mathfrak{n}_{Z}(g, h)\right) \\
& \quad \times \dot{v}\left(\mathfrak{n}_{N}(h, k)\right)\left(\left[\frac{x}{T^{\prime}}\right]-\left[\frac{x-\{\mathrm{m}(g)\}_{T^{\prime}}}{T^{\prime}}\right]\right)
\end{aligned}
$$

Therefore we obtain the image of $c$ under the map $\partial$ by evaluating at 0 :

$$
\begin{aligned}
c(\bar{p}, \bar{q}, \bar{r})= & c(\pi(g), \pi(h), \pi(k))(0) \dot{v}\left(\mathfrak{n}_{N}(h, k)\right)^{-\left[\frac{-\{\mathrm{m}(g)\rangle_{T^{\prime}}}{T^{\prime}}\right]} ; \\
(\partial c)(g, h, k)=c( & \pi(g), \pi(h), \pi(k))(0) \dot{v}\left(\mathrm{n}_{N}(k)\right)^{-\mathfrak{n}_{Z}(g, h)} \\
& \times \dot{v}\left(\mathfrak{n}_{N}(h, k)\right)^{-\left[\frac{-(\mathrm{m}(g)\rangle_{T^{\prime}}}{T^{\prime}}\right]} \\
= & c(\bar{p}, \bar{q}, \bar{r}) \dot{v}\left(\mathrm{n}_{N}(k)\right)^{-\mathfrak{n}_{Z}(g, h)} .
\end{aligned}
$$

Summarizing the above arguments, we describe the modified HJR-exact sequence of [17, Theorem 2.7] in terms of cohomology groups with the coefficient group $\mathrm{T}$ in the following: 
THEOREM 3.11. There is a commutative diagram between the modified Huebschmann-Jones-Ratcliffe exact sequences of $\widetilde{H}$ and $Q_{\mathrm{m}}$ :

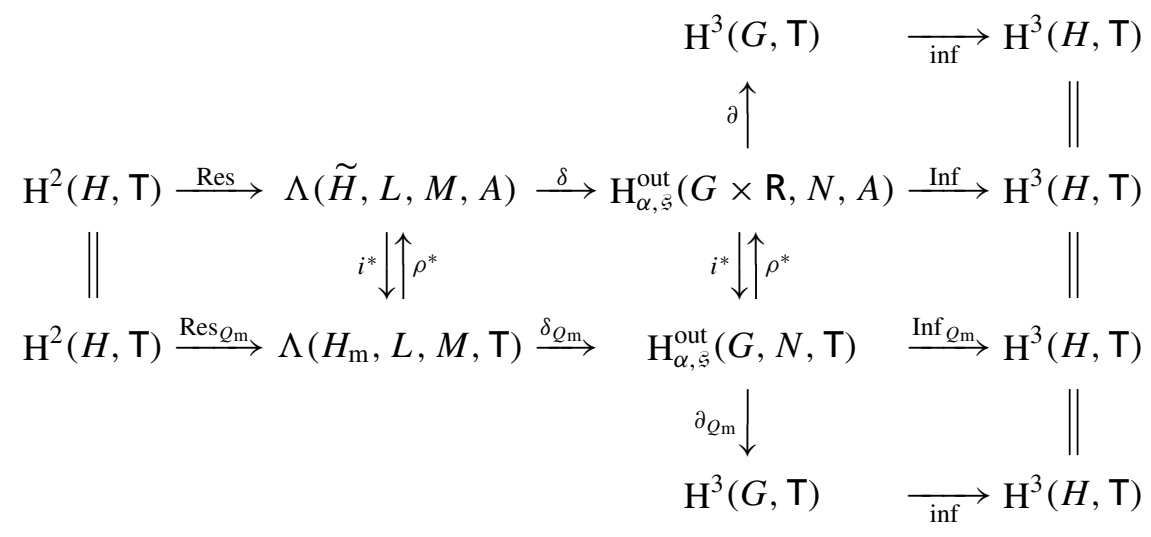

where the maps related to the group $Q_{\mathrm{m}}$ are indexed by $Q_{\mathrm{m}}$.

Definition 3.12. The second four term exact sequence:

$$
\mathrm{H}^{2}(H, \mathrm{~T}) \stackrel{\operatorname{Res}_{Q_{\mathrm{m}}}}{\longrightarrow} \Lambda\left(H_{\mathrm{m}}, L, M, \mathrm{~T}\right) \stackrel{\delta_{Q_{\mathrm{m}}}}{\longrightarrow} \mathrm{H}_{\alpha, \mathfrak{G}}^{\text {out }}(G, N, \mathrm{~T}) \stackrel{\operatorname{Inf}_{Q_{\mathrm{m}}}}{\longrightarrow} \mathrm{H}^{3}(H, \mathrm{~T})
$$

will be called the reduced modified Huebschmann-Jones-Ratcliffe exact sequence or simply the reduced modified HJR-exact sequence ([11], [12], [21]).

REMARK 3.13. The advantage of the reduced modified HJR-exact sequence over the non-reduced one is that all the groups involved are obviously compact, while the non-compactness of the coefficient group $A$ in the non reduced one forces us to prove the compactness of the cohomology group by examining the group of cocycles and coboundaries.

\section{Outer actions of a Countable Discrete Amenable Group on an AFD Factor of Type III $_{\lambda}, 0<\lambda<1$}

We first apply the result of the last section to the outer automorphism group $\operatorname{Out}(\mathcal{M})$ by taking $\operatorname{Out}(\mathcal{M})$ as $G$ and $\mathrm{H}_{\theta}^{1}\left(\mathrm{R}, \mathcal{U}\left(L^{\infty}\left(\mathrm{R} / T^{\prime} \mathrm{Z}\right)\right)\right) \cong \mathrm{R} / T \mathrm{Z}$ as $N$ :

THeORem 4.1. Suppose that $\mathcal{M}$ is a separable factor of type $\mathrm{III}_{\lambda}, 0<\lambda<1$. The intrinsic modular invariant $\mathrm{Ob}_{\mathrm{m}}(\mathcal{M})$ lives in the group:

$$
\mathrm{Ob}_{\mathrm{m}}(\mathcal{M})=\left([c], v_{\mathcal{M}}\right) \in \mathrm{H}_{\mathrm{m}, \mathfrak{S}}^{\mathrm{out}}\left(\operatorname{Out}(\mathcal{M}), \mathrm{H}_{\theta}^{1}, \mathrm{~T}\right),
$$

where $\mathrm{H}_{\theta}^{1}$ is the image $\left\{\dot{\sigma}_{s}: s \in \mathbf{R}\right\}$ of the modular automorphism group $\left\{\sigma_{s}^{\varphi}: s \in \mathrm{R}\right\}, \varphi \in \mathfrak{W}_{0}(\mathcal{M})$, in the quotient group $\operatorname{Out}(\mathcal{M})=\operatorname{Aut}(\mathcal{M}) / \operatorname{Int}(\mathcal{M})$, $\nu_{\mathcal{M}}$ is the identity map of $\mathrm{H}_{\theta}^{1}$ onto itself, and $\mathrm{m}$ is the modulus map $\mathrm{m}: \dot{\alpha} \in$ 
$\operatorname{Out}(\mathcal{M}) \mapsto \bmod (\dot{\alpha}) \in \mathrm{R} / T^{\prime}$ Z. The group $\mathrm{H}_{\mathrm{m}, \mathfrak{S}}^{\text {out }}\left(\operatorname{Out}(\mathcal{M}), \mathrm{H}_{\theta}^{1}, \mathrm{~T}\right)$ is a nonseparable compact abelian group.

In view of [17, Theorem 3.2], there is nothing left for the validity of the assertion. But we want to identify the cocycle $c \in \mathrm{H}_{\mathrm{s}}^{3}\left(\mathrm{Out}_{\tau, \theta}(\widetilde{\mathcal{M}})_{\mathrm{m}}, \mathrm{T}\right)$ directly, where

$$
\operatorname{Out}_{\tau, \theta}(\tilde{\mathcal{M}})_{\mathrm{m}}=\left\{(p, s) \in \operatorname{Out}_{\tau, \theta}(\tilde{\mathcal{M}}) \times \mathrm{R}: \mathrm{m}(p)=\dot{s}\right\} .
$$

Before going further, let us fix notations for quotient maps:

$$
\begin{gathered}
\pi: \operatorname{Out}(\mathcal{M}) \mapsto \operatorname{Out}_{\tau, \theta}(\tilde{\mathcal{M}}), \\
\tilde{\pi}: \operatorname{Aut}(\mathcal{M}) \mapsto \operatorname{Out}_{\tau, \theta}(\tilde{\mathcal{M}})=\operatorname{Aut}(\mathcal{M}) / \operatorname{Cnt}_{\mathrm{r}}(\mathcal{M}), \\
\pi_{0}: \operatorname{Aut}(\mathcal{M}) \mapsto \operatorname{Out}(\mathcal{M})=\operatorname{Aut}(\mathcal{M}) / \operatorname{Int}(\mathcal{M}), \\
\tilde{\pi}=\pi \circ \pi_{0} .
\end{gathered}
$$

Fix a generalized trace $\psi \in \mathfrak{S}_{0}(\mathcal{M})$ so that $\sigma_{T}^{\psi}=$ id. The one-parameter unitary group $\left\{\psi^{i t}: t \in \mathbf{R}\right\}$ generates a von Neumann algebra $\mathscr{A}^{\psi}$ isomorphic to $L^{\infty}(\mathrm{R})$ and the non-commutative flow $\theta$ restricted to $\mathcal{A}^{\psi}$ is identified with the translation $\rho$ :

$$
\left(\rho_{t} f\right)(x)=f(x+t), \quad f \in L^{\infty}(\mathbf{R}), \quad x, t \in \mathbf{R} .
$$

We identify $\mathscr{A}^{\psi}$ and $L^{\infty}(\mathrm{R})$ and $\psi$ is then given by the function:

$$
\psi(x)=e^{-x}, \quad x \in \mathbf{R} .
$$

The center $\mathcal{C}$ of $\widetilde{\mathcal{M}}$ is then represented by the fixed point subalgebra $\left(\mathcal{A}^{\psi}\right)^{\theta_{T^{\prime}}}$ of $\mathcal{A}^{\psi}$, i.e., the subalgebra of periodic functions with period $T^{\prime}$. We refer [28, Exercise XII.6, p. 455] for detail.

Lemma 4.2. If $\psi \in \mathfrak{S}_{0}(\mathcal{M})$ is a generalized trace, i.e., a faithful semifinite normal weight with period $T$ and $\psi(1)=\infty$, then $\mathcal{M}$ and $u_{\psi}\left(\dot{s}_{T}\right)=$ $b_{\psi}\left(\mathfrak{S}_{\mathrm{Z}}\left(\dot{s}_{T}\right)\right), s \in \mathbf{R}$, generates the discrete core $\widetilde{\mathcal{M}}_{d}$.

i) The periodic one parameter unitary group $\left\{u_{\psi}\left(\dot{s}_{T}\right): s \in \mathbf{R}\right\}$ is represented by the following function after $\mathcal{A}^{\psi}$ is identified with $L^{\infty}(\mathrm{R})$ :

$$
u_{\psi}(\dot{s} ; x)=\exp \left(\mathrm{i} T^{\prime}\{\dot{s}\}_{T}\left[\frac{x}{T^{\prime}}\right]\right)=\left\langle\dot{s},\left[\frac{x}{T^{\prime}}\right]\right\rangle, \quad x \in \mathrm{R}, \dot{s} \in \mathrm{R} / T \mathrm{Z},
$$

which is also represented as a function of $\psi$ in the following form:

$$
u_{\psi}(s)=\exp \left(\mathrm{i} T^{\prime} s\left[\frac{-\log \psi}{T^{\prime}}\right]\right)=\sum_{n \in \mathbf{Z}} \lambda^{-\mathrm{i} n s} \chi_{\left(\lambda^{n+1}, \lambda^{n}\right]}(\psi), \quad s \in \mathbf{R}
$$


where $\chi_{\left(\lambda^{n+1}, \lambda^{n}\right]}(\psi)$ means the spectral projection of $\psi$ corresponding to the half open interval $\left(\lambda^{n+1}, \lambda^{n}\right]$.

ii) The cocycle $\mathfrak{s}_{\mathrm{Z}}(\dot{s})$ is cobounded by $u_{\psi}(\dot{s})$ in $U\left(\mathcal{A}^{\psi}\right)$ relative to $\theta$ :

$$
\mathfrak{s}_{\mathrm{Z}}(\dot{s}, t ; x)=\left\langle\dot{s},\left[\frac{x+t}{T^{\prime}}\right]-\left[\frac{x}{T^{\prime}}\right]\right\rangle, \quad \dot{s} \in \mathrm{R} / T \mathrm{Z}, x \in \mathrm{R}
$$

iii) $\operatorname{Aut}(\mathcal{M})_{\mathrm{m}}$ acts on the discrete core $\widetilde{\mathcal{M}}_{d}$, i.e., if $(\alpha, s) \in \operatorname{Aut}(\mathcal{M})_{\mathrm{m}}$, then $\theta_{s} \circ \alpha$ leaves $\widetilde{\mathcal{M}}_{d}$ globally invariant.

Proof. The claims (i) and (ii) follow directly from [10] with sign change in the coboundary operation $\partial_{\theta}$. So we prove only (iii). First, observe that if $(\alpha, s) \in \operatorname{Aut}(\mathcal{M})_{\mathrm{m}}$, then $\theta_{s} \circ \alpha$ acts on the center $\mathcal{C}$ trivially, i.e., it acts as the identity since the actions $\bmod (\alpha)$ and $\theta_{s}$ cancel each other on $\mathcal{C}$. Hence there exists $u \in U(\mathcal{M})$ such that $\theta_{s} \circ \alpha(\psi)=u \psi u^{*}$. Thus we get

$$
\begin{aligned}
\theta_{s} \circ \alpha\left(u_{\psi}\left(\dot{s}_{T}\right)\right) & =\theta_{s} \circ \alpha\left(\sum_{n \in \mathrm{Z}} \lambda^{-\mathrm{i} n s} \chi_{\left(\lambda^{n+1}, \lambda^{n}\right]}(\psi)\right) \\
& =\sum_{n \in \mathrm{Z}} \lambda^{-\mathrm{i} n s} \chi_{\left(\lambda^{n+1}, \lambda^{n}\right]}\left(\theta_{s} \circ \alpha(\psi)\right) \\
& =\sum_{n \in \mathrm{Z}} \lambda^{-\mathrm{i} n s} \chi_{\left(\lambda^{n+1}, \lambda^{n}\right]}\left(u \psi u^{*}\right) \\
& =u\left(\sum_{n \in \mathrm{Z}} \lambda^{-\mathrm{i} n s} \chi_{\left(\lambda^{n+1}, \lambda^{n}\right]}(\psi)\right) u^{*} \\
& =u u_{\psi}\left(\dot{s}_{T}\right) u^{*} .
\end{aligned}
$$

Hence $\theta_{s} \circ \alpha\left(u_{\psi}\left(\dot{s}_{T}\right)\right) \in \mathcal{M} \vee\left\{u_{\psi}\left(\dot{s}_{T}\right): \dot{s}_{T} \in \mathrm{R} / T \mathrm{Z}\right\}^{\prime \prime}=\widetilde{\mathcal{M}}_{d}$. This completes the proof.

The generalized trace $\psi$ gives rise to the semi-direct product decomposition:

$$
\mathrm{Cnt}_{\mathrm{r}}(\mathcal{M})=\operatorname{Int}(\mathcal{M}) \rtimes_{\sigma^{\psi}}(\mathrm{R} / T \mathrm{Z}) .
$$

We are going to use the notation $\sigma_{\dot{s}}, \dot{s} \in \mathrm{R} / T \mathrm{Z}$, for the element of $\mathrm{Cnt}_{\mathrm{r}}(\mathcal{M})$ corresponding to an element $\dot{s}=\dot{s}_{T}=s+T \mathrm{Z} \in \mathrm{R} / T \mathrm{Z}$.

LemMA 4.3. i) It is possible to choose a cross-section $u: m \in \mathrm{Cnt}_{\mathrm{r}}(\mathcal{M}) \mapsto$ $u(m) \in \widetilde{U}_{0}(\mathcal{M}) \subset \widetilde{\mathcal{M}}_{d}$ such that $u(m) \in \mathcal{U}(\mathcal{M})$ if $m \in \operatorname{Int}(\mathcal{M})$ and

$$
u\left(\sigma_{\dot{s}} m\right)=u_{\psi}(\dot{s}) u(m), \quad \dot{s} \in \mathrm{R} / T \mathrm{Z}, \quad m \in \mathrm{Cnt}_{\mathrm{r}}(\mathcal{M}) .
$$

ii) There exists a cross-section $g \in \operatorname{Out}(\mathcal{M}) \mapsto \alpha_{g} \in \operatorname{Aut}(\mathcal{M})$ such that 
a) Each $\alpha_{g}, g \in \operatorname{Out}(\mathcal{M})$, transforms $\psi$ to a scalar multiple of $\psi$, i.e., $\alpha_{g}(\psi)$ and $\psi$ are proportional. Consequently $\alpha_{g}$ and $\sigma^{\psi}$ commute;

b)

$$
\alpha_{\sigma_{\dot{s}} g}=\sigma_{\dot{s}}^{\psi} \circ \alpha_{g}, \quad \dot{s} \in \mathrm{R} / T \mathrm{Z} .
$$

c) The associated $\operatorname{Int}(\mathcal{M})$-valued cocycle $\eta_{\text {in }}$ has the property:

$$
\eta_{\text {in }}\left(\sigma_{\dot{s}} g, \sigma_{i} h\right)=\eta_{\text {in }}(g, h), \quad g, h \in \operatorname{Out}(\mathcal{M}), \quad \dot{s}, \dot{t} \in \mathrm{R} / T \mathrm{Z},
$$

so that it is the pullback $\pi^{*}\left(\eta_{Q}\right)$ of an $\operatorname{Int}(\mathcal{M})$-valued two cochain $\eta_{Q} \in$ $\mathrm{C}_{\alpha}^{2}\left(\mathrm{Out}_{\tau, \theta}(\widetilde{\mathcal{M}}), \operatorname{Int}(\mathcal{M})\right)$.

Proof. i) First choose a Borel cross-section $u: m \in \operatorname{Int}(\mathcal{M}) \mapsto u(m) \in$ $\mathcal{U}(\mathcal{M})$ of the adjoint map $\operatorname{Ad}: v \in \mathcal{U}(\mathcal{M}) \mapsto \operatorname{Ad}(v) \in \operatorname{Int}(\mathcal{M})$. Then extend the cross-section by setting:

$$
u\left(\sigma_{\dot{s}} m\right)=u_{\psi}(\dot{s}) u(m), \quad \dot{s} \in \mathrm{R} / T \mathbf{Z}, \quad m \in \operatorname{Int}(\mathcal{M}) .
$$

This gives a cross-section with the desired property.

ii) For any $\alpha \in \operatorname{Aut}(\mathcal{M}), \alpha(\psi)$ is another generalized trace on $\mathcal{M}$. Hence there exists a scalar $\mu \in \operatorname{R}$ and a unitary $v \in \mathcal{U}(\mathcal{M})$ such that $\operatorname{Ad}(v) \circ \alpha(\psi)=$ $\mu \psi$. In fact, $\mu$ is can be chosen to be $e^{\{\bmod (\alpha)\}_{T^{\prime}}}$. Hence it is possible to a representative $\alpha_{g}$ of $g \in \operatorname{Out}(\mathcal{M})$. With this in mind, we select first a crosssection $p \in \operatorname{Out}_{\tau, \theta}(\tilde{\mathcal{M}}) \mapsto \alpha_{p} \in \operatorname{Aut}(\mathcal{M})$ of the quotient map $\tilde{\pi}: \operatorname{Aut}(\mathcal{M}) \mapsto$ $\operatorname{Out}_{\tau, \theta}(\widetilde{\mathcal{M}})=\operatorname{Aut}(\mathcal{M}) / \operatorname{Cnt}_{\mathrm{r}}(\mathcal{M})$ such that

a) The weights $\alpha_{p}(\psi)$ and $\psi$ are proportional, i.e., $\alpha_{p}(\psi)=e^{\{\bmod (p)\}_{T^{\prime}}} \psi$;

b) The quotient map $\pi_{0}: \operatorname{Aut}(\mathcal{M}) \mapsto \operatorname{Aut}(\mathcal{M}) / \operatorname{Int}(\mathcal{M})$ maps $\alpha_{p}$ exactly into the cross-section image $\mathfrak{s}(p) \in \operatorname{Out}(\mathcal{M})$ of $p$ which has been fixed already.

The cross-section $\alpha$ generates a $\operatorname{Cnt}_{\mathrm{r}}(\mathcal{M})$-valued two cocycle:

$$
\eta_{\alpha}(p, q)=\alpha_{p} \circ \alpha_{q} \circ \alpha_{p q}^{-1} \in \mathrm{Cnt}_{\mathrm{r}}(\mathcal{M}), \quad p, q \in \operatorname{Out}_{\tau, \theta}(\tilde{\mathcal{M}}) .
$$

Then we have

$$
\begin{aligned}
\pi_{0}\left(\eta_{\alpha}(p, q)\right) & =\pi_{0}\left(\alpha_{p}\right) \pi\left(\alpha_{q}\right) \pi_{0}\left(\alpha_{p q}^{-1}\right)=\mathfrak{s}(p) \mathfrak{s}(q) \mathfrak{s}(p q)^{-1} \\
& =\mathfrak{n}_{\mathfrak{s}}(p, q), \quad p, q \in \operatorname{Out}_{\tau, \theta}(\tilde{\mathcal{M}}) .
\end{aligned}
$$

Therefore, the semi-direct product decomposition: $\operatorname{Cnt}_{\mathrm{r}}(\mathcal{M})=\operatorname{Int}(\mathcal{M}) \rtimes_{\sigma^{\psi}}$ $\mathrm{R} / T \mathrm{Z}$ gives a decomposition of $\eta_{\alpha}$ :

$$
\eta_{\alpha}(p, q)=\mathfrak{n}_{\mathfrak{\xi}}(p, q) \eta_{\text {in }}(p, q), \quad p, q \in \operatorname{Out}_{\tau, \theta}(\tilde{\mathcal{M}}),
$$


where $\mathfrak{n}_{\mathfrak{s}}(p, q) \in \mathrm{H}_{\theta}^{1}$ and $\eta_{\text {in }}(p, q) \in \operatorname{Int}(\mathcal{M})$ commutes with $\left\{\sigma_{s}^{\psi} ; s \in \mathrm{R}\right\}$. Now decomposing each $g \in \operatorname{Out}(\mathcal{M})$ in the form:

$$
g=\mathfrak{m}_{\mathrm{H}_{\theta}^{1}}(g) \mathfrak{S}(\pi(g)),
$$

and writing $\dot{s}(g) \in \mathrm{R} / T \mathrm{Z}$ for $\mathfrak{m}_{\mathrm{H}_{\theta}^{1}}(g) \in \mathrm{H}_{\theta}^{1}$, we set

$$
\alpha_{g}=\sigma_{\dot{s}(g)}^{\psi} \circ \alpha_{\pi(g)} \in \operatorname{Aut}_{\psi}^{\prime}(\mathcal{M})=\left\{\alpha \in \operatorname{Aut}(\mathcal{M}): \alpha \circ \sigma_{s}^{\psi}=\sigma_{s}^{\psi} \circ \alpha, s \in \mathrm{R}\right\} .
$$

Observing for each pair $g, h \in \operatorname{Out}(\mathcal{M})$ that

$$
\begin{aligned}
& g h=\mathfrak{m}_{\mathrm{H}_{\theta}^{1}}(g) \mathfrak{S}(\pi(g)) \mathfrak{m}_{\mathrm{H}_{\theta}^{1}}(h) \mathfrak{S}(\pi(h)) \\
&=\mathfrak{m}_{\mathrm{H}_{\theta}^{1}}(g) \mathfrak{m}_{\mathrm{H}_{\theta}^{1}}(h) \mathfrak{S}(\pi(g)) \mathfrak{s}(\pi(h)), \quad \text { as } \mathrm{H}_{\theta}^{1} \subset \text { Center of Out }(\mathcal{M}), \\
&=\mathfrak{m}_{\mathrm{H}_{\theta}^{1}}(g) \mathfrak{m}_{\mathrm{H}_{\theta}^{1}}(h) \mathfrak{H}_{\mathfrak{S}}(\pi(g), \pi(h)) \mathfrak{s}(\pi(g h)) \\
&=\mathfrak{m}_{\mathrm{H}_{\theta}^{1}}(g h) \mathfrak{S}(\pi(g h)) ; \\
& \mathfrak{m}_{\mathrm{H}_{\theta}^{1}}(g) \mathfrak{m}_{\mathrm{H}_{\theta}^{1}}(h)=\mathfrak{m}_{\mathrm{H}_{\theta}^{1}}(g h) \mathfrak{H}_{\mathfrak{F}}(\pi(g), \pi(h))^{-1},
\end{aligned}
$$

we compute:

$$
\begin{aligned}
\alpha_{g} \circ \alpha_{h} & =\sigma_{\dot{s}(g)}^{\psi} \circ \alpha_{\pi(g)} \circ \sigma_{\dot{s}(h)}^{\psi} \circ \alpha_{\pi(h)}=\sigma_{\dot{s}(g)+\dot{s}(h)}^{\psi} \circ \alpha_{\pi(g)} \circ \alpha_{\pi(h)} \\
& =\sigma_{\dot{s}(g)+\dot{s}(h)}^{\psi} \circ \eta_{\alpha}(\pi(g), \pi(h)) \circ \alpha_{\pi(g h)} \\
& =\sigma_{\dot{s}(g h)}^{\psi} \circ \mathfrak{n}_{\xi}(\pi(g), \pi(h))^{-1} \circ \eta_{\alpha}(\pi(g), \pi(h)) \circ \alpha_{\pi(g h)} \\
& =\eta_{\text {in }}(\pi(g), \pi(h)) \circ \sigma_{\dot{s}(g h)}^{\psi} \circ \alpha_{\pi(g h)} \\
& =\eta_{\text {in }}(\pi(g), \pi(h)) \circ \alpha_{g h}
\end{aligned}
$$

Therefore, the map $\alpha: g \in \operatorname{Out}(\mathcal{M}) \mapsto \alpha_{g} \in \operatorname{Aut}_{\psi}^{\prime}(\mathcal{M})$ is indeed an outer action of Out $(\mathcal{M})$. Furthermore, $\eta_{\text {in }}(\pi(g), \pi(h))$ belongs to the group $\operatorname{Int}(\mathcal{M}) \cap$ $\operatorname{Aut}^{\prime}(\mathcal{M})$ which is given by the normalizer $N\left(\mathcal{M}_{\psi}\right)$ of the centralizer $\mathcal{M}_{\psi}$ of $\psi$. From its construction, $\eta_{\text {in }}$ satisfies the requirement of the lemma. This completes the proof.

Before going into the last step, we need the following:

Lemma 4.4. i) If $\alpha \in \operatorname{Aut}_{\psi}^{\prime}(\mathcal{M})$, then $\alpha$ leaves $\psi$ relatively invariant, so that we have $\mathrm{k} \in \operatorname{Hom}\left(\operatorname{Aut}_{\psi}^{\prime}(\mathcal{M}), \mathrm{R}\right)$ such that

$$
\alpha(\psi)=e^{\mathrm{k}(\alpha)} \psi, \quad \alpha \in \operatorname{Aut}_{\psi}^{\prime}(\mathcal{M}),
$$

and $\alpha$ and $\theta_{-\mathrm{k}(\alpha)}$ agree on $\mathcal{A}^{\psi}$, in particular on $u_{\psi}(\dot{s}), \dot{s} \in \mathrm{R} / T \mathrm{Z}$; 
ii) If $(\alpha, s) \in \operatorname{Aut}_{\psi}^{\prime}(\mathcal{M})_{\mathrm{m}}$, then

$$
\left(\left(\alpha \circ \theta_{s}\right)\left(u_{\psi}(\dot{t})\right) u_{\psi}(\dot{t})^{*}\right)(x)=\left\langle\dot{t}, \frac{s-\mathrm{k}(\alpha)}{T^{\prime}}\right\rangle
$$

in particular the left hand side is constant in $x \in \mathrm{R}$, i.e., the unitary $u_{\psi}(\dot{t}), \dot{t} \in$ $\mathrm{R} / T \mathrm{Z}$, is an eigen operator of $\alpha \circ \theta_{s}$.

Proof. If $\alpha$ is in $\operatorname{Aut}_{\psi}^{\prime}(\mathcal{M})$, then its extension to $\widetilde{\mathcal{M}}$, still denoted by $\alpha$, leaves $\mathcal{A}^{\psi}$ globally invariant and

$$
(\alpha f)(x)=f(x-\mathrm{k}(\alpha)), \quad f \in \mathcal{A}^{\psi}, \quad x \in \mathrm{R},
$$

as seen below:

$$
(\alpha \psi)(x)=e^{\mathrm{k}(\alpha)} \psi(x)=e^{\mathrm{k}(\alpha)} e^{-x}=e^{-(x-\mathrm{k}(\alpha))}=\psi(x-\mathrm{k}(\alpha)) .
$$

The center $\mathcal{C}$ of $\widetilde{\mathcal{M}}$ is generated by $\psi^{\mathrm{i} T}$, so that it is identified with the subalgebra of periodic functions with period $T^{\prime}$, i.e., $\mathcal{C}=\left(\mathcal{A}^{\psi}\right)^{\theta_{T^{\prime}}}$. By Lemma 4.2, the periodic one parameter unitary group:

$$
\left\{u_{\psi}\left(\dot{s}_{T}\right): \dot{s}_{T} \in \mathrm{R} / T \mathrm{Z}\right\}=\left\{b_{\psi}\left(\mathfrak{S}_{\mathrm{Z}}\left(\dot{s}_{T}\right)\right): \dot{s}_{T} \in \mathrm{R} / T \mathrm{Z}\right\}
$$

is represented in $\mathcal{A}^{\psi}$ by the following functions:

$$
u_{\psi}\left(\dot{s}_{T} ; x\right)=\exp \left(\mathrm{i} T^{\prime} s\left[\frac{x}{T^{\prime}}\right]\right), \quad x, s \in \mathrm{R}
$$

which together with $\mathcal{M}$ generates the discrete core $\widetilde{\mathcal{M}}_{d}$. Since $\alpha \in \operatorname{Aut}_{\psi}^{\prime}(\mathcal{M})$ and $\theta_{-\mathrm{k}(\alpha)}$ both scales the generator $\psi$ of $\mathcal{A}^{\psi}$ in the same way, they agree on $\mathcal{A}^{\psi}$. Hence we get

$$
\begin{aligned}
\left(\alpha\left(u_{\psi}(\dot{s})\right)\right)(x) & =u_{\psi}(\dot{s} ; x-\mathrm{k}(\alpha)) \\
& =\exp \left(\mathrm{i} T^{\prime}\{\dot{s}\}_{T}\left[\frac{x-\mathrm{k}(\alpha)}{T^{\prime}}\right]\right), \quad \dot{s} \in \mathrm{R} / T \mathbf{Z} .
\end{aligned}
$$

If $(\alpha, s) \in \operatorname{Aut}_{\psi}^{\prime}(\mathcal{M})_{\mathrm{m}}$, then

$$
\begin{aligned}
\left(\alpha \circ \theta_{s}\right)\left(u_{\psi}(\dot{t})\right)(x) & =u_{\psi}(\dot{t} ; x+s-\mathrm{k}(\alpha)) \\
& =\exp \left(\mathrm{i} T^{\prime}\{\dot{t}\}_{T}\left[\frac{x+s-\mathrm{k}(\alpha)}{T^{\prime}}\right]\right)
\end{aligned}
$$


and

$$
\begin{aligned}
\left(\alpha \circ \theta_{s}\right)\left(u_{\psi}(\dot{t})\right) u_{\psi}(\dot{t})^{*}(x) & =u_{\psi}\left(\dot{t} ; x+s-\mathrm{k}(\alpha) \overline{u_{\psi}(\dot{t} ; x)}\right. \\
& =\exp \left(\mathrm{i} T^{\prime}\{\dot{t}\}_{T}\left(\left[\frac{x+s-\mathrm{k}(\alpha)}{T^{\prime}}\right]-\left[\frac{x}{T^{\prime}}\right]\right)\right) \\
& =\exp \left(\mathrm{i} T^{\prime}\{\dot{t}\}_{T} \frac{s-\mathrm{k}(\alpha)}{T^{\prime}}\right)=\left\langle\dot{t}, \frac{s-\mathrm{k}(\alpha)}{T^{\prime}}\right\rangle,
\end{aligned}
$$

where the last pairing makes sense because $s-\mathrm{k}(\alpha) \in T^{\prime} \mathrm{Z}$ for $(\alpha, s) \in$ $\operatorname{Aut}_{\psi}^{\prime}(\mathcal{M})_{\mathrm{m}}$. This completes the proof.

Now the next lemma completes the proof of Theorem 4.1 .

LEMmA 4.5. If a cross-section $u: m \in \mathrm{Cnt}_{\mathrm{r}}(\mathcal{M}) \mapsto u(m) \in \widetilde{\mathcal{U}}_{0}(\mathcal{M})$ is the one given by Lemma 4.3, then the natural choices of $u(g, h), g, h \in \operatorname{Out}(\mathcal{M})_{\mathrm{m}}$, and $u(\tilde{p}, \tilde{q}), \tilde{p}=(p, s), \tilde{q}=(q, t) \in \operatorname{Out}_{\tau, \theta}(\widetilde{\mathcal{M}})_{\mathrm{m}}, b y$

$$
u(g, h)=u\left(\eta_{\text {in }}(g, h)\right) \in \mathcal{U}(\mathcal{M}) \quad \text { and } \quad u(\tilde{p}, \tilde{q})=u\left(\eta_{\alpha}(p, q)\right) \in \tilde{U}_{0}(\mathcal{M})
$$

give the following:

i)

$$
\begin{aligned}
\alpha_{g} \circ \alpha_{h} & =\operatorname{Ad}(u(g, h)) \circ \alpha_{g h} \\
\alpha_{\tilde{p}} \circ \alpha_{\tilde{q}} & =\operatorname{Ad}(u(\tilde{p}, \tilde{q})) \circ \alpha_{\tilde{p} \tilde{q}}
\end{aligned}
$$

ii) The associated cocycles $c_{\mathcal{M}}^{\mathrm{o}} \in \mathrm{Z}^{2}(\operatorname{Out}(\mathcal{M}), \mathrm{T})$ given by:

$$
\begin{aligned}
& c_{\mathcal{M}}^{\circ}(g, h, k) \\
& \quad=\alpha_{g}(u(h, k)) u(g, h k)\{u(g, h) u(g h, k)\}^{*}, \quad g, h, k \in \operatorname{Out}(\mathcal{M}),
\end{aligned}
$$

gives the cohomology class $\left[c_{\mathcal{M}}^{\circ}\right] \in \mathrm{H}^{3}(\operatorname{Out}(\mathcal{M}), \mathrm{T})$ which is the intrinsic obstruction $\mathrm{Ob}(\mathcal{M})$.

iii) The cocycle $c_{\mathcal{M}}$ associated with the choice of $u$ :

$$
\begin{aligned}
& c_{\mathcal{M}}(\tilde{p}, \tilde{q}, \tilde{r}) \\
& \quad=\alpha_{\tilde{p}}(u(\tilde{q}, \tilde{r})) u(\tilde{p}, \tilde{q} \tilde{r})\{u(\tilde{p}, \tilde{q}) u(\tilde{p} \tilde{q}, \tilde{r})\}^{*}, \quad \tilde{p}, \tilde{q}, \tilde{r} \in \operatorname{Out}_{\tau, \theta}(\tilde{\mathcal{M}})_{\mathrm{m}}
\end{aligned}
$$

is a standard cocycle relative to the distinguished central element $z_{0}=$ $\left(1, T^{\prime}\right) \in \operatorname{Out}_{\tau, \theta}(\widetilde{\mathcal{M}})_{\mathrm{m}}$ and the d-part is given by:

$$
d(q, r)=\exp \left(\mathrm{iT}^{\prime}\left\{\mathfrak{n}_{\mathfrak{S}}(q, r)\right\}_{T^{\prime}}\right), \quad q, r \in \mathrm{Out}_{\tau, \theta}(\tilde{\mathcal{M}}),
$$

so that

$$
c_{\mathcal{M}}\left(\tilde{p} z_{0}^{m}, \tilde{q} z_{0}^{n}, \tilde{r} z_{0}^{\ell}\right)=\left\langle\mathfrak{n}_{\xi}(q, r), m\right\rangle c_{\mathcal{M}}(\tilde{p}, \tilde{q}, \tilde{r}),
$$

for each $m, n, \ell \in \mathrm{Z}$ and $\tilde{p}, \tilde{q}, \tilde{r} \in \mathrm{Out}_{\tau, \theta}(\tilde{\mathcal{M}})_{\mathrm{m}}$. 
iv) The pair $\left(c_{\mathcal{M}}\right.$, id) belongs to $\mathrm{Z}_{\mathrm{s}}^{3}\left(\operatorname{Out}_{\tau, \theta}(\mathcal{M})_{\mathrm{m}}, \mathrm{T}\right) *_{\xi} \operatorname{Hom}_{\text {Out }(M)}\left(\mathrm{H}_{\theta}^{1}, \mathrm{H}_{\theta}^{1}\right)$ and its cohomology class $\left[c_{\mathcal{M}}, \mathrm{id}\right]$ in $\mathrm{H}_{\mathrm{s}, \mathrm{m}}^{\mathrm{out}}\left(\mathrm{Out}(\mathcal{M}), \mathrm{H}_{\theta}^{1}, \mathrm{~T}\right)$ is in fact the intrinsic modular obstruction $\mathrm{Ob}_{\mathrm{m}}(\mathcal{M})$.

Proof. We see immediately for each pair $g, h \in \operatorname{Out}(\mathcal{M})$

$$
\begin{aligned}
\alpha_{g} \circ \alpha_{h} & =\eta_{\text {in }}(g, h) \circ \alpha_{g h}=\operatorname{Ad}\left(u\left(\eta_{\text {in }}(g, h)\right)\right) \circ \alpha_{g h} \\
& =\operatorname{Ad}(u(g, h)) \circ \alpha_{g h},
\end{aligned}
$$

and for each pair $\tilde{p}=(p, s), \tilde{q}=(q, t) \in \operatorname{Out}_{\tau, \theta}(\tilde{\mathcal{M}})_{\mathrm{m}}$

$$
\begin{aligned}
\alpha_{\tilde{p}} \circ \alpha_{\tilde{q}} & =\theta_{s} \circ \alpha_{p} \circ \theta_{t} \circ \alpha_{q}=\theta_{s+t} \circ \alpha_{p} \circ \alpha_{q}=\theta_{s+t} \circ \eta_{\alpha}(p, q) \circ \alpha_{p q} \\
& =\widetilde{\operatorname{Ad}}\left(u\left(\eta_{\alpha}(p, q)\right)\right) \circ \alpha_{\tilde{p} \tilde{q}}=\widetilde{\operatorname{Ad}}(u(p, q)) \circ \alpha_{\tilde{p} \tilde{q}} .
\end{aligned}
$$

Consequently, $c_{\mathcal{M}}^{\circ}$ and $c_{\mathcal{M}}$ are both three cocycles and the former gives the intrinsic obstruction $\mathrm{Ob}(\mathcal{M})$ in the cohomology group $\mathrm{H}^{3}(\operatorname{Out}(\mathcal{M}), \mathrm{T})$. To see the standardness of $c_{\mathcal{M}}$, we just examine:

$$
\begin{aligned}
c_{\mathcal{M}}\left(\tilde{p} z_{0}^{m}, \tilde{q} z_{0}^{n}, \tilde{r} z_{0}^{\ell}\right) \\
=\alpha_{\tilde{p}} \circ \theta_{m T^{\prime}}\left(u\left(\tilde{q} z_{0}^{n}, \tilde{r} z_{0}^{\ell}\right)\right) u\left(\tilde{p} z_{0}^{m}, \tilde{r} z_{0}^{n+\ell}\right) \\
\quad \times\left\{u\left(\tilde{p} z_{0}^{m}, \tilde{q} z_{0}^{n}\right) u\left(\tilde{p} \tilde{q} z_{0}^{m+n}, \tilde{r} z_{0}^{\ell}\right)\right\}^{*} \\
=\alpha_{\tilde{p}} \circ \theta_{m T^{\prime}}(u(q, r)) u(p, q r)\{u(p, q) u(p q, r)\}^{*} \\
=\alpha_{\tilde{p}} \circ \theta_{m T^{\prime}}\left(u_{\psi}\left(\mathfrak{n}_{\mathfrak{\xi}}(q, r)\right) u\left(\eta_{\text {in }}(q, r)\right)\right) u(p, q r)\{u(p, q) u(p q, r)\}^{*} \\
=\left\langle\mathfrak{n}_{\mathfrak{\xi}}(q, r), m\right\rangle \alpha_{\tilde{p}}\left(u_{\psi}\left(\mathfrak{n}_{\mathfrak{\xi}}(q, r)\right) u\left(\eta_{\mathrm{in}}(q, r)\right)\right) \\
\quad \times u(p, q r)\{u(p, q) u(p q, r)\}^{*} \\
=\left\langle\mathfrak{n}_{\mathfrak{\xi}}(q, r), m\right\rangle \alpha_{\tilde{p}}(u(q, r)) u(p, q r)\{u(p, q) u(p q, r)\}^{*} \\
=\left\langle\mathfrak{n}_{\mathfrak{\xi}}(q, r), m\right\rangle c_{\mathcal{M}}(\tilde{p}, \tilde{q}, \tilde{r}) .
\end{aligned}
$$

Since the $d$-part is given by the two cocycle $\mathfrak{n}_{\mathfrak{S}}$ itself, the pair $\left(c_{\mathcal{M}}\right.$, id) belongs to the fiber product $Z^{3}\left(\operatorname{Out}_{\tau, \theta}(\mathcal{M})_{\mathrm{m}}, \mathrm{T}\right) *_{\xi} \operatorname{Hom}_{\mathrm{Out}(M)}\left(\mathrm{H}_{\theta}^{1}, \mathrm{H}_{\theta}^{1}\right)$.

THEOREM 4.6. i) If $G$ is a discrete group and $\alpha$ is an outer action of $G$ on a factor $\mathcal{M}$ of type $\mathrm{III}_{\lambda}, 0<\lambda<1$, then the modulus $\mathrm{m}=\mathrm{m}_{\alpha}: g \in G \mapsto$ $\bmod (g) \in \mathrm{R} / T \mathbf{Z}^{\prime}$ of $\alpha$, the normal subgroup $N=N(\alpha)=\alpha^{-1}\left(\mathrm{Cnt}_{\mathrm{r}}(\mathcal{M})\right)$, a homomorphism $v_{\alpha}: m \in N \mapsto \dot{\alpha}_{m} \in \mathrm{H}_{\theta}^{1} \cong \mathrm{R} / T \mathrm{Z}$ and the "pullback" $\left[c_{\alpha}\right]=\alpha^{*}\left(\left[c_{\mathcal{M}}\right]\right) \in \mathrm{H}_{\mathrm{m}, \mathcal{F}}^{\text {out }}(G, N, \mathrm{~T})$ of the intrinsic modular obstruction, to be termed the modular obstruction of $\alpha$, are outer conjugacy invariants of $\alpha$. 
ii) If $G$ is a countable discrete amenable group and the factor $\mathcal{M}$ of type $\mathrm{III}_{\lambda}$ is approximately finite dimensional, then the invariants $\left\{\mathrm{m}_{\alpha}, N(\alpha),\left[c_{\alpha}\right], v_{\alpha}\right\}$ determines the outer conjugacy class of $\alpha$. The group $\mathrm{H}_{\mathrm{m}, \mathfrak{S}}^{\mathrm{out}}(G, N, \mathrm{~T})$ is a separable compact abelian group.

REMARK 4.7. The pullback in the theorem needs a qualification. As the cross-section $\mathfrak{S}: \operatorname{Out}_{\tau, \theta}(\widetilde{\mathcal{M}}) \mapsto \operatorname{Out}(\mathcal{M})$ is only guaranteed by the axiom of choice, we have no idea if it consistent with the map $\dot{\alpha}: G \mapsto \operatorname{Out}(\mathcal{M})$, for example it can happen that $\mathfrak{S}\left(\mathrm{Out}_{\tau, \theta}(\widetilde{\mathcal{M}})\right) \cap \dot{\alpha}(G)=\{$ id $\}$. Namely, we cannot pull back the cross-section $\mathfrak{S}$ of $\operatorname{Out}_{\tau, \theta}(\widetilde{\mathcal{M}})$. So we have to work with a cross-section $\mathfrak{g}: Q=G / N \mapsto G$ directly. But this does not change the picture concerning the modular obstruction $\mathrm{Ob}_{\mathrm{m}}(\alpha)$. If we consider all crosssections of $\operatorname{Out}_{\tau, \theta}(\widetilde{\mathcal{M}})$ and form the $\operatorname{group} \mathrm{H}^{\text {out }}\left(\operatorname{Out}(\mathcal{M}), \mathrm{H}_{\theta}^{1}, \mathrm{~T}\right)$ as in $[17$, p. 218], in which we locate the intrinsic modular obstruction, then one can pull back $\operatorname{Ob}_{\mathrm{m}}(\mathcal{M})$ to form the modular obstruction $\operatorname{Ob}_{\mathrm{m}}(\alpha) \in \mathrm{H}^{\text {out }}(G, N, \mathrm{~T})$ since cross-section of $Q_{\widetilde{M}} \mapsto G$ can be carried to $\operatorname{Out}_{\tau, \theta}(\widetilde{\mathcal{M}})$ as a part of a cross-section of $\operatorname{Out}_{\tau, \theta}(\widetilde{\mathcal{M}})$, i.e., we can have a cross-section $\mathfrak{S}$ of $\operatorname{Out}_{\tau, \theta}(\widetilde{\mathcal{M}})$ so that $\dot{\alpha}^{-1}\left(\mathfrak{S}\left(\mathrm{Out}_{\tau, \theta}(\widetilde{\mathcal{M}})\right) N=G\right.$, which enables us to pull back the structure concerning $\operatorname{Aut}(\mathcal{M}), \operatorname{Out}(\mathcal{M})$ and $\operatorname{Out}_{\tau, \theta}(\widetilde{\mathcal{M}})$.

THEOREM 4.8. Suppose that $\mathcal{M}$ is a factor of type $\mathrm{III}_{\lambda}, 0<\lambda<1$, and that $\psi$ is a periodic faithful semi-finite normal weight with period $T=$ $-2 \pi / \log \lambda$. Let $\mathrm{m}: \alpha \in \operatorname{Aut}(\mathcal{M}) \mapsto \bmod (\alpha) \in \mathrm{R} / T^{\prime} \mathbf{Z}=\operatorname{Aut}_{\theta}(\mathcal{C})$ be the modulus homomorphism of $\operatorname{Aut}(\mathcal{M})$ to the automorphism group $\operatorname{Aut}_{\theta}(\mathcal{C})$ of the flow of weights $\{\mathcal{C}, \mathrm{R}, \theta\}=\left\{L^{\infty}\left(\mathrm{R} / T^{\prime} \mathrm{Z}\right)\right.$, Translation $\}$ which is identified with the quotient group $\mathrm{R} / T^{\prime} \mathrm{Z}$, then the discrete core $\widetilde{\mathcal{M}}_{d}$ gives rise to an $\operatorname{Aut}(\mathcal{M})_{\mathrm{m}}$-equivariant commutative square of exact sequences:

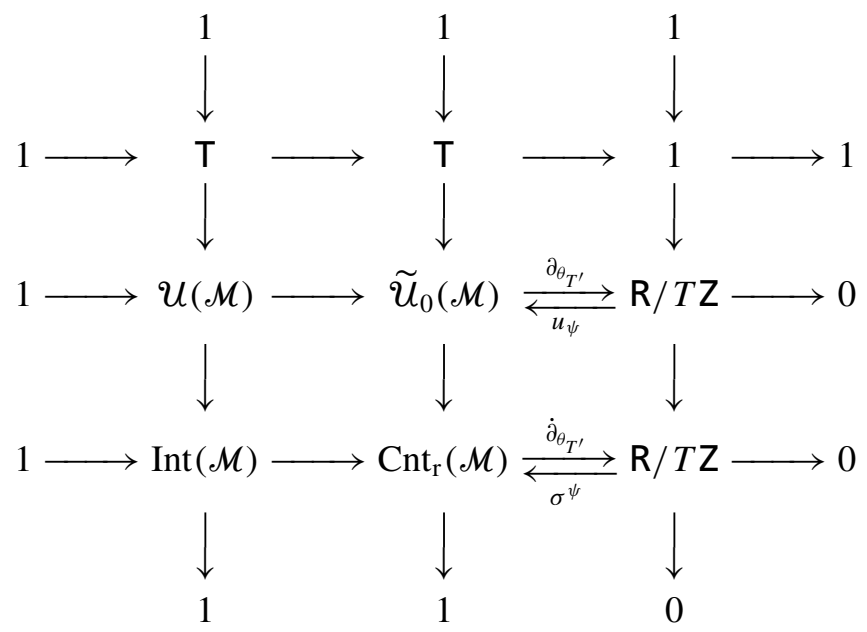


where $\tilde{U}_{0}(\mathcal{M})$ is the unitary normalizer of $\mathcal{M}$ in the discrete core $\widetilde{\mathcal{M}}_{d}$, i.e.,

$$
\tilde{\mathcal{U}}_{0}(\mathcal{M})=\tilde{U}(\mathcal{M}) \cap \widetilde{\mathcal{M}}_{d}=\left\{u \in \mathcal{U}\left(\widetilde{\mathcal{M}}_{d}\right): u \mathcal{M} u^{*}=\mathcal{M}\right\} .
$$

This is an easy consequence of the previous discussion, in particular Lemma 4.2.iii and so we leave the proof to the reader.

Definition 4.9. The above exact square is called the reduced characteristic square of $\mathcal{M}$.

\section{Outer actions of a Countable Discrete Amenable Group on an AFD Factor of Type III $_{1}$}

The triviality of the flow of weights on a factor of type $\mathrm{III}_{1}$ makes the characteristic square very simple:

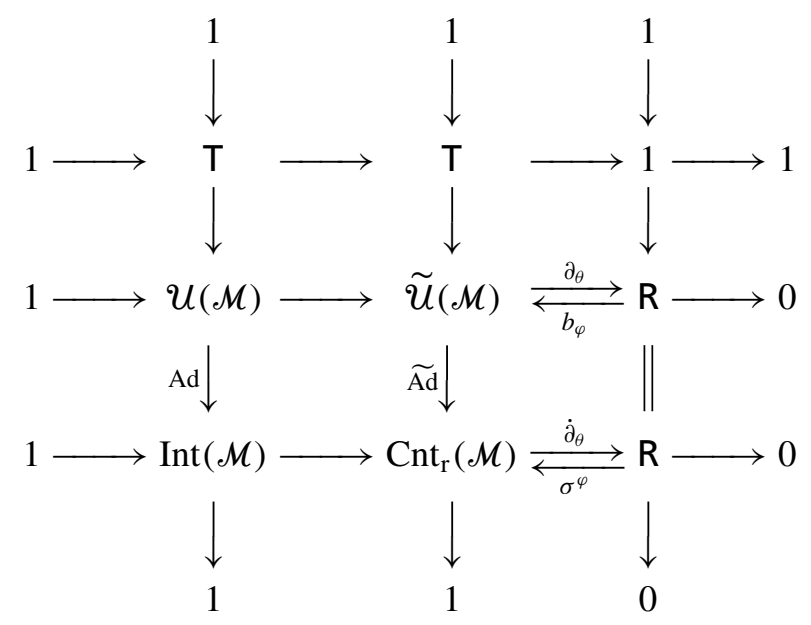

Furthermore, the horizontal exact sequences split nicely. When we view $R$ as a central subgroup of $\operatorname{Out}(\mathcal{M})$, we denote it by $\mathrm{H}_{\theta}^{1}$. We will identify $\mathrm{H}_{\theta}^{1}$ and $\mathrm{R}$ frequently to avoid heavy notations in the case of type $\mathrm{III}_{1}$.

THeOREM 5.1. Let $\mathcal{M}$ be a factor of type $\mathrm{III}_{1}$. Fix a cross-section

$$
\mathfrak{g}_{\pi}: p \in \operatorname{Out}_{\tau, \theta}(\tilde{\mathcal{M}}) \mapsto \mathfrak{g}_{\pi}(p) \in \operatorname{Out}(\mathcal{M})
$$

of the quotient map $\pi: \operatorname{Out}(\mathcal{M}) \mapsto \operatorname{Out}_{\tau, \theta}(\tilde{\mathcal{M}})=\operatorname{Out}(\mathcal{M}) / \mathrm{H}_{\theta}^{1}$ and the associated R-valued cocycle:

$$
\mathfrak{n}_{\mathrm{R}}(p, q)=\mathfrak{s}(p) \mathfrak{s}(q) \mathfrak{S}(p q)^{-1} \in \mathrm{R} \cong \mathrm{H}_{\theta}^{1}, \quad p, q \in \operatorname{Out}_{\tau, \theta}(\tilde{\mathcal{M}}) .
$$


It is possible to select cross-sections $\alpha: g \in \operatorname{Out}(\mathcal{M}) \mapsto \alpha_{g} \in \operatorname{Aut}(\mathcal{M})$ and $u: m \in \operatorname{Cnt}_{\mathrm{r}}(\mathcal{M}) \mapsto u(m) \in \widetilde{U}(\mathcal{M})$ so that

i) $m=\widetilde{\operatorname{Ad}}(u(m)), m \in \operatorname{Cnt}_{\mathrm{r}}(\mathcal{M})$;

ii) the associated intrinsic modular obstruction cocycle $c_{\mathcal{M}}$ takes the form:

$$
c_{\mathcal{M}}(\tilde{p}, \tilde{q}, \tilde{r})=\exp \left(-\mathrm{i} s \mathfrak{H}_{\mathrm{R}}(q, r)\right) c_{\mathrm{Out}_{\tau, \theta}(\mathcal{M})}(p, q, r),
$$

for each $\tilde{p}=(p, s), \tilde{q}, \tilde{r} \in \mathrm{Out}_{\tau, \theta}(\tilde{\mathcal{M}}) \times \mathrm{R}$.

Let $\alpha$ be an outer action of a countable discrete group $G$ on $\mathcal{M}$ with $N=$ $\alpha^{-1}\left(\operatorname{Cnt}_{\mathrm{r}}(\mathcal{M})\right)$ and $\nu_{\alpha}(m)=\dot{\alpha}_{m} \in \mathrm{R} \cong \mathrm{H}_{\theta}^{1}, m \in N$. Fix a cross-section s: $Q=G / N \mapsto G$ of the quotient map $\pi: G \mapsto Q$ along with the associated $N$-valued cocycle:

$$
\mathfrak{n}_{N}(p, q)=\mathfrak{s}(p) \mathfrak{s}(q) \mathfrak{s}(p q)^{-1} \in N, \quad p, q \in Q .
$$

Then the pullback cocycle $c_{\alpha}$ of $c_{\mathcal{M}}$ by $\alpha^{*}$ takes the form:

$$
c_{\alpha}(\tilde{p}, \tilde{q}, \tilde{r})=\exp \left(-\mathrm{i} s v_{\alpha}\left(\mathfrak{n}_{N}(q, r)\right)\right) c_{Q}(p, q, r),
$$

for each $\tilde{p}=(p, s), \tilde{q}, \tilde{r} \in \widetilde{Q}=Q \times$ R. Its cohomology class $\left(\left[c_{\alpha}\right], v_{\alpha}\right) \in$ $\mathrm{H}_{\mathfrak{g}}^{\text {out }}(G, N, \mathrm{~T})$ is the modular obstruction $\mathrm{Ob}_{\mathrm{m}}(\alpha)$ of $\alpha$.

Proof. Fix a dominant weight $\varphi$ on $\mathcal{M}$ and observe that

$$
\operatorname{Aut}(\mathcal{M})=\operatorname{Int}(\mathcal{M}) \operatorname{Aut}_{\varphi}(\mathcal{M}), \quad \operatorname{Aut}_{\varphi}(\mathcal{M})=\{\alpha \in \operatorname{Aut}(\mathcal{M}): \varphi \circ \alpha=\varphi\} .
$$

Then we have

$$
\begin{aligned}
\operatorname{Out}(\mathcal{M}) & =\operatorname{Aut}(\mathcal{M}) / \operatorname{Int}(\mathcal{M})=\operatorname{Aut}_{\varphi}(\mathcal{M}) /\left(\operatorname{Aut}_{\varphi}(\mathcal{M}) \cap \operatorname{Int}(\mathcal{M})\right), \\
\operatorname{Out}_{\tau, \theta}(\mathcal{M}) & =\operatorname{Aut}(\mathcal{M}) / \operatorname{Cnt}_{\mathrm{r}}(\mathcal{M})=\operatorname{Aut}_{\varphi}(\mathcal{M}) /\left(\operatorname{Aut}_{\varphi}(\mathcal{M}) \cap \operatorname{Cnt}_{\mathrm{r}}(\mathcal{M})\right), \\
\tilde{U}(\mathcal{M}) & =\mathcal{U}(\mathcal{M}) \rtimes_{\sigma^{\varphi}} \operatorname{R.} .
\end{aligned}
$$

The invariance $\varphi=\varphi \circ \widetilde{\operatorname{Ad}}(u), u \in \widetilde{U}(\mathcal{M})$, of $\varphi$ gives the decomposition $u=v \varphi^{\mathrm{i} s}$ for some $v \in \mathcal{U}\left(\mathcal{M}_{\varphi}\right)$ and $s \in \mathrm{R}$. Hence we get the decomposition:

$$
\widetilde{\operatorname{Ad}}^{-1}\left(\operatorname{Cnt}_{\mathrm{r}}(\mathcal{M}) \cap \operatorname{Aut}_{\varphi}(\mathcal{M})\right) \cong \mathcal{U}\left(\mathcal{M}_{\varphi}\right) \times \mathrm{R} .
$$

Therefore, we can choose a cross-section $p \in \operatorname{Out}_{\tau, \theta}(\mathcal{M}) \mapsto \alpha_{p} \in \operatorname{Aut}_{\varphi}(\mathcal{M})$ such that

$\alpha_{p} \circ \alpha_{q}=\operatorname{Ad}(v(p, q)){ }^{\circ} \sigma_{\mathfrak{l n}_{\mathrm{R}}(p, q)}^{\varphi}{ }^{\circ} \alpha_{p q}, \quad v(p, q) \in U\left(\mathcal{M}_{\varphi}\right), \quad p, q \in \operatorname{Out}_{\tau, \theta}(\mathcal{M})$.

We then set

$$
\alpha_{g}=\sigma_{\mathrm{s}(g)} \circ \alpha_{\pi(g)}, \quad g \in \operatorname{Out}(\mathcal{M}),
$$


where

$$
g=\sigma_{\mathrm{s}(g)}^{\varphi} \mathfrak{S}_{\pi}(\pi(g)) .
$$

Setting

$$
u(\tilde{p}, \tilde{q})=v(p, q) \varphi^{\mathrm{in}_{\mathrm{R}}(p, q)}, \quad \tilde{p}=(p, s), \quad \tilde{q}=(q, t) \in \mathrm{Out}_{\tau, \theta}(\mathcal{M}) \times \mathrm{R},
$$

we compute the intrinsic modular obstruction cocycle:

$$
\begin{aligned}
c_{\mathcal{M}}(\tilde{p}, \tilde{q}, \tilde{r}) & =\alpha_{\tilde{p}}(u(\tilde{q}, \tilde{r})) u(\tilde{p} \tilde{q}, \tilde{r})\{u(\tilde{p}, \tilde{q}) u(\tilde{p} \tilde{q}, \tilde{r})\}^{*} \\
& =\alpha_{p} \circ \theta_{s}(u(q, r)) u(p q, r)\{u(p, q) u(p q, r)\}^{*} \\
& =\exp \left(-\mathrm{i} s \mathfrak{n}_{\mathrm{R}}(q, r)\right) \alpha_{p}(u(q, r)) u(p, q r)\{u(p, q) u(p q, r)\}^{*} \\
& =\exp \left(-\mathrm{i} s \mathfrak{n}_{\mathrm{R}}(q, r)\right) \alpha_{p}(v(q, r)) v(p, q r)\{v(p, q) v(p q, r)\}^{*},
\end{aligned}
$$

where the last step follows from the fact that $\mathfrak{n}_{\mathrm{R}}$ is an $\mathrm{R}$-valued two cocycle over Out $_{\tau, \theta}(\mathcal{M})$. Therefore, we conclude that

$$
c_{\mathcal{M}}(\tilde{p}, \tilde{q}, \tilde{r})=\exp \left(-\mathrm{i} s \mathfrak{n}_{\mathrm{R}}(q, r)\right) c_{\operatorname{Out}_{\tau, \theta}(\mathcal{M})}(p, q, r) .
$$

Now we look at $\alpha_{g} \circ \alpha_{h}, g, h \in \operatorname{Out}(\mathcal{M})$ :

$$
\begin{aligned}
\alpha_{g} \circ \alpha_{h} & =\sigma_{\mathrm{s}(g)}^{\varphi} \circ \alpha_{\mathfrak{F}_{\pi}(\pi(g))} \circ \sigma_{\mathrm{s}(h)}^{\varphi} \circ \alpha_{\mathfrak{F}_{\pi}(\pi(h))} \\
& =\sigma_{\mathrm{s}(g) \mathrm{s}(h)}^{\varphi} \circ \alpha_{\mathfrak{F}_{\pi}(\pi(h))} \circ \alpha_{\mathfrak{F}_{\pi}(\pi(h))} \\
& =\sigma_{\mathrm{s}(g) \mathrm{s}(h)}^{\varphi} \circ \widetilde{A d}(u(\pi(g), \pi(h))) \circ \alpha_{\mathfrak{F}_{\pi}(\pi(g h))} \\
& =\sigma_{\mathrm{s}(g) \mathrm{s}(h)}^{\varphi} \circ \sigma_{\mathfrak{n}_{\mathrm{R}}(\pi(g), \pi(h))}^{\varphi} \circ \operatorname{Ad}\left(v(\pi(g), \pi(h)) \circ \alpha_{\mathfrak{F}_{\pi}(\pi(g h))} .\right.
\end{aligned}
$$

Also we observe:

$$
\begin{aligned}
g h & =\mathrm{s}(g) \mathfrak{S}_{\pi}(\pi(g)) \mathrm{s}(h) \mathfrak{S}_{\pi}(\pi(h))=\mathrm{s}(g) \mathrm{s}(h) \mathfrak{S}_{\pi}(\pi(g)) \mathfrak{S}_{\pi}(\pi(h)) \\
& =\mathrm{s}(g) \mathrm{s}(h) \mathfrak{H}_{\mathrm{H}_{\theta}^{1}}(\pi(g), \pi(h)) \mathfrak{G}_{\pi}(\pi(g h)) \\
& =\mathrm{s}(g h) \mathfrak{S}_{\pi}(\pi(g h)), \quad g, h \in \operatorname{Out}(\mathcal{M}) ; \\
& \mathrm{s}(g)+\mathrm{s}(h)=\mathrm{s}(g h)-\mathfrak{n}_{\mathrm{R}}(\pi(g), \pi(h)) .
\end{aligned}
$$

Plugging this into the previous computation, we get

$$
\alpha_{g} \circ \alpha_{h}=\operatorname{Ad}\left(v(\pi(g), \pi(h)) \circ \alpha_{g h}, \quad g, h \in \operatorname{Out}(\mathcal{M}) .\right.
$$

Therefore, the intrinsic obstruction cocycle $c$ is given by the pullback:

$$
c(g, h, k)=c_{\operatorname{Out}_{\tau, \theta}(\mathcal{M})}(\pi(g), \pi(h), \pi(k)), \quad g, h, k \in \operatorname{Out}(\mathcal{M}),
$$

of the restriction of the intrinsic modular obstruction cocycle $c_{\widetilde{\mathcal{M}}}$ to the sub$\operatorname{group~Out}_{\tau, \theta}(\mathcal{M})$. This completes the proof. 


\section{REFERENCES}

1. Brylinski, J. L., and Nistor, V., Cyclic cohomology of etale groupoids, K-Theory 8 (1994), 341-365.

2. Connes, A., Une classification des facteurs de type III, Ann. Sci. École Norm. Sup. (4) 6 (1973), 133-252.

3. Connes, A., Almost periodic states and factors of type $\mathrm{III}_{1}$, J. Funct. Anal. 16 (1974), 415-445.

4. Connes, A., Periodic automorphisms of the hyperfinite factor of type $\mathrm{II}_{1}$, Acta Math. Szeged 39 (1977), 39-66.

5. Connes, A., Outer conjugacy of automorphisms of factors, Sympos. Math. 20, 149-159.

6. Connes, A., Classification of injective factors, Ann. of Math. 104 (1976), 73-115.

7. Connes, A., Outer conjugacy classes of automorphisms of factors, Ann. Sci. École Norm. Sup. (4) 8 (1975), 383-419.

8. Connes,A., and Takesaki, M., The flow of weights on factors of type III, Tôhoku Math. J. 29 (1977), 473-575.

9. Eilenberg, S., and MacLane, S., Cohomology theory in abstract groups, Ann. of Math. 48 (1947), 51-78.

10. Falcone, A. J., and Takesaki, M., Non-commutative flow of weights on a von Neumann algebra, J. Funct. Anal. 182 (2001), 170-206.

11. Huebschmann, J., Group extensions, crossed pairs and an eight term exact sequence, J. Reine Angew. Math. 321 (1981), 150-172.

12. Jones, V. F. R., Actions of finite groups on the hyperfinite type III factor, Mem. Amer. Math. Soc. 237 (1980).

13. Jones, V. F. R., and Takesaki, M., Actions of compact abelian groups on semifinite injective factors, Acta Math. 153 (1984), 213-258.

14. Katayama, Y., Sutherland, C. E., and Takesaki, M., The characteristic square of a factor and the cocycle conjugacy of discrete amenable group actions on factors, Invent. Math. 132 (1998), 331-380.

15. Katayama, Y., Sutherland, C. E., and Takesaki, M., The structure of the automorphism group of a factor and cocycle conjugacy of discrete group actions, Proceeding of Conference on Operator Algebras and Quantum Field Theory, International Press, Cambridge, MA, 1997, 166-198.

16. Katayama, Y., and Takesaki, M., Outer actions of a countable discrete amenable group on AFD factors, Contemp. Math. 335 (2003), 163-171.

17. Katayama, Y., and Takesaki, M., Outer actions of a countable discrete amenable group on approximately finite dimensional factors I, General Theory, Contemp. Math. 365 (2004), 181-237.

18. Katayama, Y., and Takesaki, M., Outer actions of a countable discrete amenable group on approximately finite dimensional factors III, Examples, in preparation.

19. Kawahigashi, Y., Sutherland, C. E., and Takesaki, M., The structure of the automorphism group of an injective factor and the cocycle conjugacy of discrete abelian group actions, Acta Math. 169 (1992), 105-130.

20. Ocneanu, A., Actions of discrete amenable groups on factors, Lecture Notes in Math. 1138 (1985).

21. Ratcliffe, J. G., Crossed extensions, Trans. Amer. Math. Soc. 237 (1980), $73-89$.

22. Sutherland, C. E., Cohomology and extensions of von Neumann algebras, I \& II, Publ. Res. Inst. Math. Sci. 16 (1980), 105-133 and 135-174.

23. Sutherland, C. E., A Borel parametrization of Polish groups, Publ. Res. Inst. Math. Sci. 21 (1985), 1067-1086.

24. Sutherland, C. E., and Takesaki, M., Actions of discrete amenable groups and groupoids on von Neumann algebras, Publ. Res. Inst. Math. Sci. 21 (1985), 1087-1120. 
25. Sutherland, C. E., and Takesaki, M., Actions of Discrete amenable groups on injective factors of type $\mathrm{III}_{\lambda}, \lambda \neq 1$, Pacific J. Math. 137 (1989), 405-444.

26. Sutherland, C. E., and Takesaki, M., Right inverse of the module of approximately finite dimensional factors of type III and approximately finite ergodic principal measured groupoids. Operator algebras and their applications II, Fields Inst. Commun. 20 (1998), 149-159.

27. Takesaki, M., Theory of Operator Algebras I, Springer-Verlag, Heidelberg, New York, Hong Kong, Tokyo, 1979.

28. Takesaki, M., Theory of Operator Algebras II, Springer-Verlag, Heidelberg, New York, Hong Kong, Tokyo, 2002.

29. Takesaki, M., Theory of Operator Algebras III, Springer-Verlag, Heidelberg, New York, Hong Kong, Tokyo, 2002.

30. Westman, J. J., Cohomology for ergodic groupoids, Trans. Amer. Math. Soc. 146 (1969), 465-471.

31. Westman, J. J., Cohomology for the ergodic actions of countable groups Proc. Amer. Math. Soc. 30 (1971), 318-320.

DEPARTMENT OF MATHEMATICS

OSAKA KYOIKU UNIVERSITY

OSAKA

JAPAN

E-mail: katayama@cc.osaka-kyoiku.ac.jp
DEPARTMENT OF MATHEMATICS UNIVERSITY OF CALIFORNIA

LOS ANGELES

USA

E-mail: mt@math.ucla.edu 
